\title{
THE EFFECTS OF VARIATION IN COLLAGEN D-SPACING ON COMPACT BONE VISCOELASTICITY: A FINITE ELEMENT ANALYSIS
}

\author{
A Thesis \\ presented to \\ the Faculty of California Polytechnic State University, \\ San Luis Obispo
}

In Partial Fulfillment

of the Requirements for the Degree

Master of Science in Biomedical Engineering

by

Miguel Angel Mendoza

August 2013 
(C) 2013

Miguel Angel Mendoza

ALL RIGHTS RESERVED 


\section{COMMITTEE MEMBERSHIP}

TITLE:

The Effects of Variation in Collagen

D-spacing on Compact Bone Viscoelasticity: A

Finite Element Analysis

AUTHOR:

Miguel Angel Mendoza

DATE SUBMITTED:

August 2013

COMMITTEE CHAIR:

Dr. Scott Hazelwood, Associate Professor, Biomedical Engineering

COMMITTEE MEMBER:

Dr. Clifford Les, Adjunct Professor, Biomedical Engineering

COMMITTEE MEMBER:

Dr. David Clague, Associate Professor, Biomedical Engineering 


\begin{abstract}
The Effects of Variation in Collagen D-spacing on Compact Bone Viscoelasticity: A Finite Element Analysis

Miguel Mendoza
\end{abstract}

The D-spacing that is characteristic of collagen and its structural arrangement was previously thought to be a constant value. Much research is revealing that it is actually a distribution of values in biological tissues. Recent ovine experimentation has also shown that the D-spacing distribution is significantly altered following estrogen depletion. While ewes contain some major biological differences between their human counterparts, they are an economical and robust large animal model for postmenopausal osteoporosis. So, the exploration of the possible implications that $\mathrm{D}$-spacing has on the mechanical properties of the whole bone utilizing animal models and computational methods is warranted. Six Warhill ewes were used in this experiment and were either ovariectomized or underwent a sham surgery. The animals were sacrificed after 3 years and the radius and ulna bone were harvested for further analysis. Rectangular beams of compact bone tissue were machined from six different sectors in the whole bone and dynamic mechanical analysis tests were performed on the 24 specimens. The viscoelastic property, tangent delta, was measured from each test at varying frequencies. A composite arrangement of collagen and hydroxyapatite were then computationally modeled utilizing finite element analysis to observe the effects of altered D-spacing on the mechanical properties. Jager and Fratzl's staggered array model allowed the inclusion of a D-spacing configuration as well as the simplified 2 dimensional plane strain analysis. Hydroxyapatite was modeled as a perfectly elastic material, while the hydrated collagen component was linear viscoelastic through the use of the standard linear solid model. The main finding of the work is that D-spacing only significantly altered the tangent delta of the computational model when the mineral volume fraction changed. Since the composite model analyzed the structural arrangement of compact bone at such a small scale, the change in mineral volume fraction could only be realistically attributed to intrafibrillar mineral. The results of this preliminary analysis are 
promising and warrant the continued investigation of D-spacing and mineral content and their significance in the osteoporotic condition.

Keywords: Postmenopausal, Osteoporosis, Ovine, D-spacing, Viscoelastic, Bone 


\section{ACKNOWLEDGMENTS}

First, I would like to thank my advisor Dr. Scott Hazelwood for the opportunity to work on this project. The courses he taught were instrumental in finding a discipline that I'm fascinated and passionate about. I would also like to thank Dr. Clifford Les for his vast knowledge in this field and invaluable guidance throughout. His ability to question and understand various processes definitely helped shape the outcome of the project. Finally, I would like to thank my friends and family. Their supportive nature has been overwhelming and I would not be where I am today without them. 


\section{TABLE OF CONTENTS}

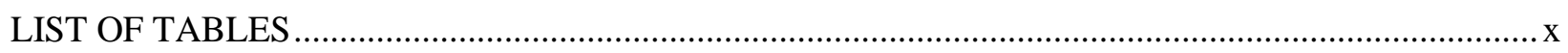

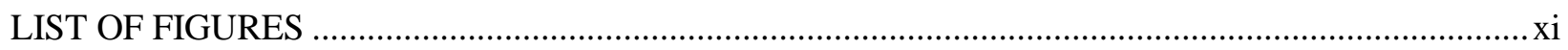

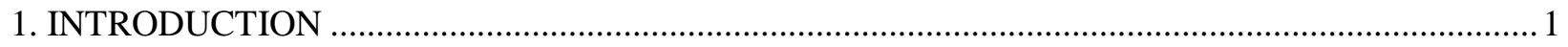

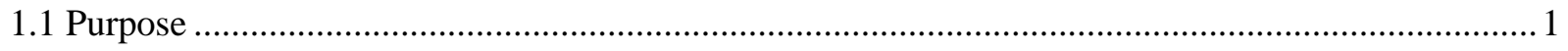

1.2 Bone

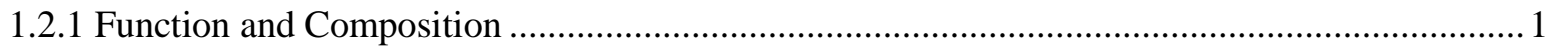

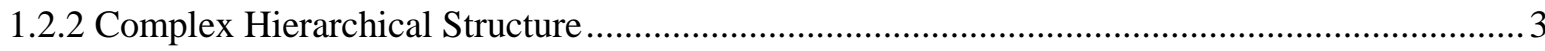

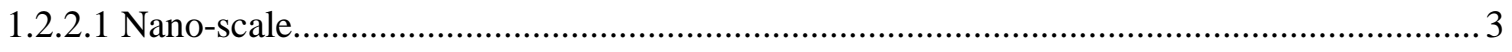

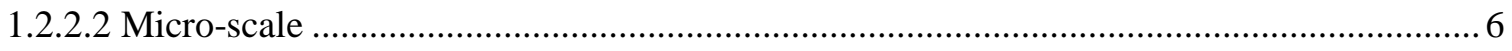

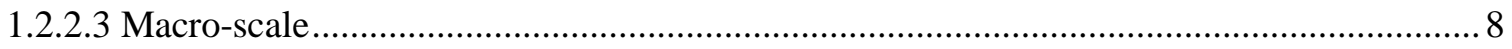

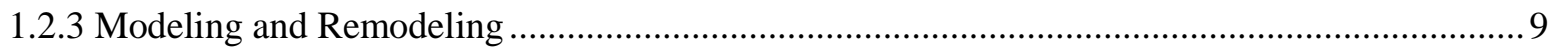

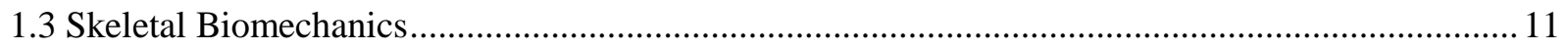

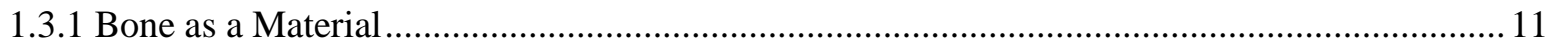

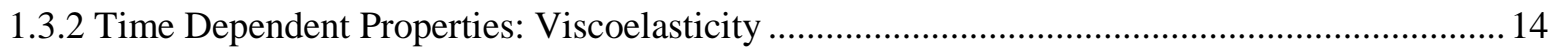

1.3.3 Modeling the Mechanical Behavior of Bone Tissue ........................................................ 16

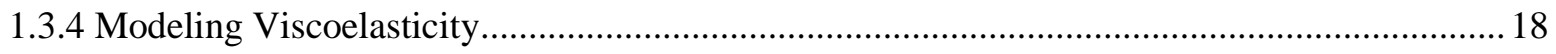

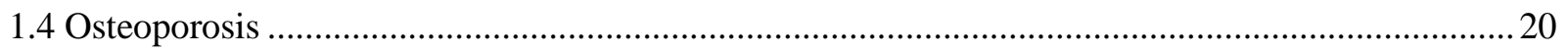

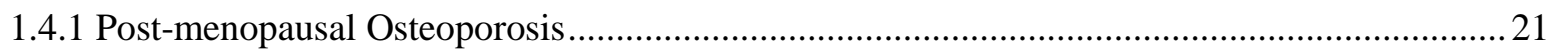

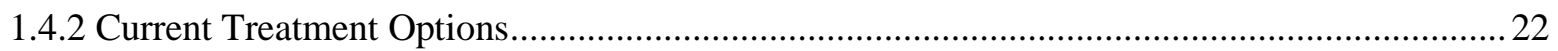

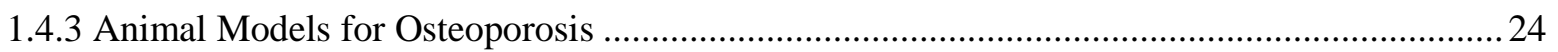




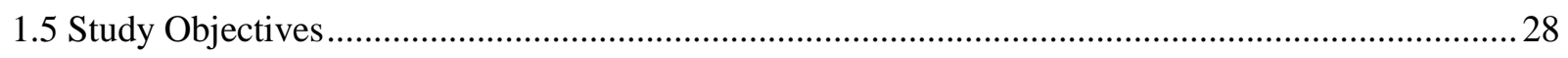

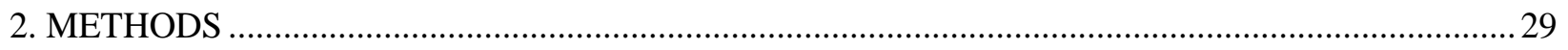

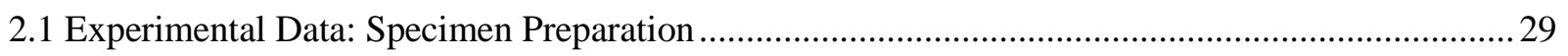

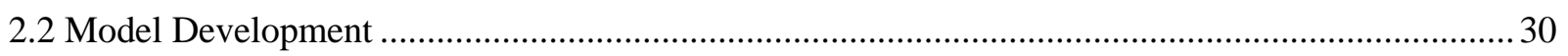

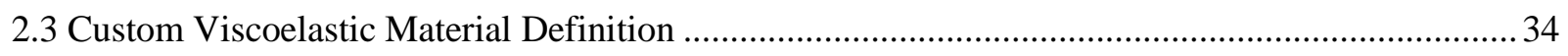

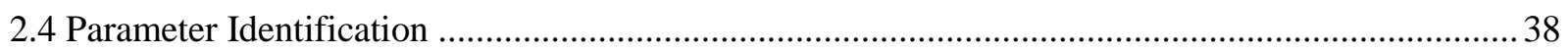

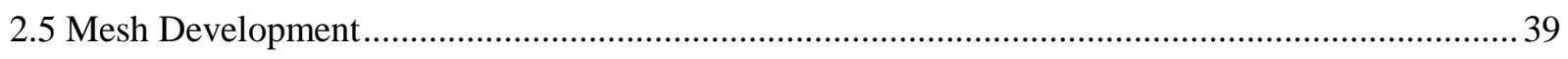

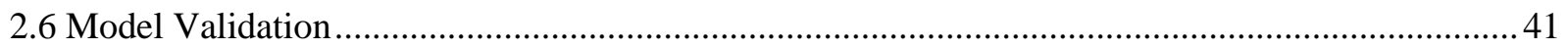

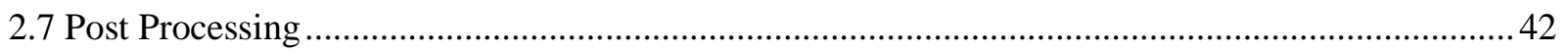

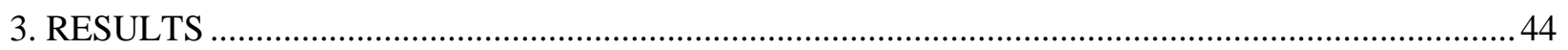

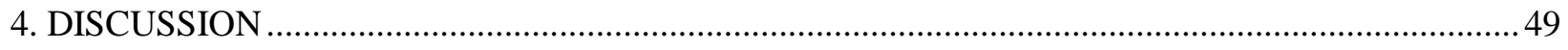

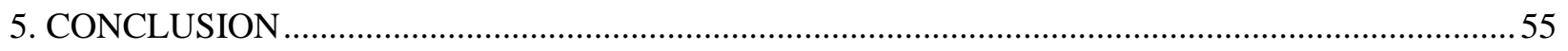

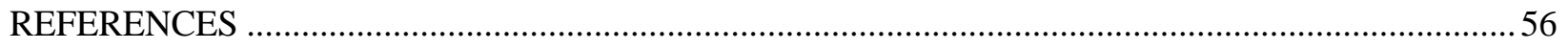

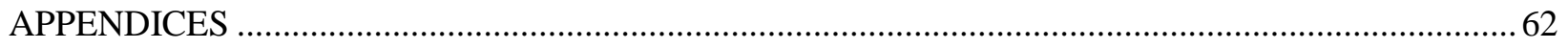

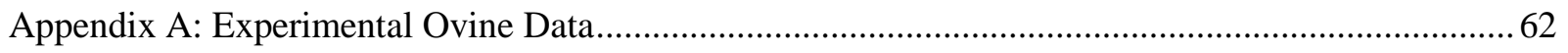

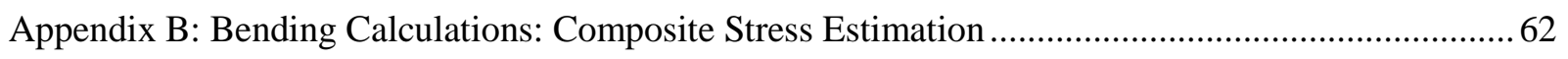

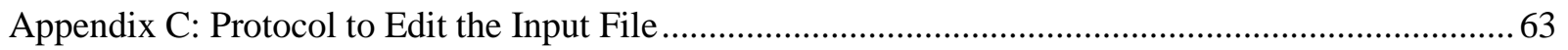

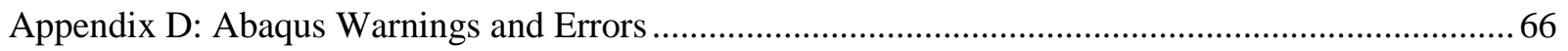

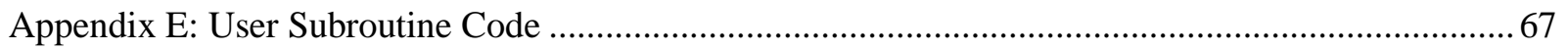

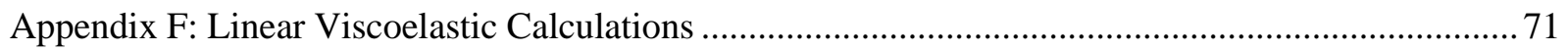




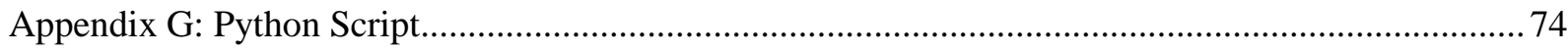

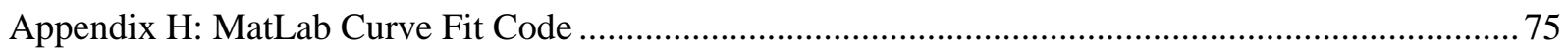




\section{LIST OF TABLES}

Table I: Elastic moduli, shear moduli, and Poisson's ratio for bone rewritten from Currey $[2,18,19]$. Units are in GPa for the moduli and the values are written in a specific ordermeanstandard deviation (sample number) —-where statistical data was available. Additionally, the subscripts 1, 2, and 3 represent the radial, circumferential, and longitudinal directions with respect to the long axis of the bone.

Table II: Elastic material properties of collagen and hydroxyapatite [1, 2, 4].

Table III: Seven model variants were developed to analyze the impact that D-spacing has on bone mechanical properties. Two of the four variables in the staggered array model were constant and are not shown (collagen width and mineral plate width). Characters in bold have deviated from the control.

Table IV: Experimental ovine DMA data at varying frequencies. The control animals underwent a sham surgery leaving the endocrine organs intact.

Table V: Composite bone FE model data for the cranial side of the ovine bone. The model for the $1 \mathrm{~Hz}$ test has a dashpot of $4 \mathrm{GPa}-\mathrm{s}$ and the model for the $15 \mathrm{~Hz}$ test has a dashpot of $1.25 \mathrm{GPa}-\mathrm{s}$. The target tangent delta originates from the experimental data.

Table VI: Composite bone FE model data for the caudal side of the ovine bone using the $1 \mathrm{GPa}-\mathrm{s}$ dashpot. The target tangent delta originates from the experimental data. 


\section{LIST OF FIGURES}

Figure 1: Hierarchical structure of bone starting from its basic composition [21] 3

Figure 2: Distribution of D-spacing values found in ovine bone [8]. 4

Figure 4: This image contains multiple Haversian systems in a field of lamellar bone. One of the osteons is incomplete and there are also various regions of osteon overlap [1].

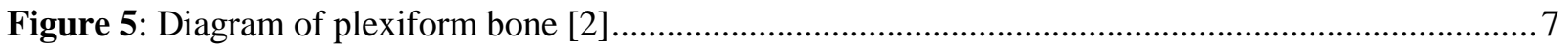

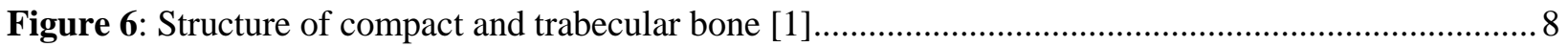

Figure 7: This diagram reveals the longitudinal cross-section of an osteon or Haversian system created by the remodeling activities of osteoblasts and osteoclasts [1].

Figure 8: Bovine bone loaded in compression at different strain rates yield different stress-strain curves. The tissue becomes stronger, stiffer, and more brittle at higher strain rates [22].

Figure 9: Stress and strain loading behaviors in a dynamic mechanical analysis test. The time difference, $\Delta t$, between the sinusoids is related to delta as shown [44]. 16

Figure 10: Comparison between various arrangements of collagen and HA. The Hodge-Petruska scheme is shown on the left and the staggered arrangement on the right. The characteristic D-spacing behavior results in overlap and gap regions that are maintained in the staggered array model [12].

Figure 11: The spring and dashpot elements are shown with their governing equations. Three simple constructions including the Kelvin-Voigt body form of the standard linear solid are also displayed [4]... 19

Figure 12: An image of normal bone on the left and osteoporotic bone on the right [52].

Figure 13: The images shown here are histological cross-sections of ewe bone. (Left) The bone is primarily composed of plexiform bone at a young age. The ewe was 3 years of age. (Right) Heavy remodeling throughout the sheep's lifetime yields Haversian bone that is very similar to adult human

cortical tissue [76]. 
Figure 14: Collagen I fibril D-spacing as a function of anatomic site and treatment. The results seem to indicate a more homogeneous distribution of D-spacing in the cortical bone tissue caused by the ovariectomy [7].

Figure 15: Cross-section of the radius and ulna bone from the ewes. The anatomical sectors are separated by gray lines.

Figure 16: a) The arrangement of collagen (light grey) in between HA crystals (black) can be seen as well as the D-spacing behavior exhibited by mineralized collagen fibrils. b) The periodic arrangement of the collagen (white) and HA (grey) are shown. c) This image shows a single cell containing regions of HA and collagen interacting with one another. 'P' represents the periodic element length $(67 \mathrm{~nm})[12,32]$; 't' represents the HA thickness $(2.5 \mathrm{~nm})$ [36]; 'L' represents the HA crystal length $(112.56 \mathrm{~nm})[36,40,47]$; 'a' represents the distance between the mineral $(21.44 \mathrm{~nm})$; 'n.d' represents three collagen triple helices between the mineral $(3 * 1.5 \mathrm{~nm}=4.5 \mathrm{~nm})$. d) The final model where HA is in dark grey and the white represents the collagen. .30

Figure 17: (a) Normal D-spacing model with red highlighted region indicates the mineral crystal. The gray regions represent the collagen domains. (b) The coordinate axis is shown in yellow at the far end of the model. (c) The boundary conditions and loads can be seen on the composite bone model. This model is on the tensile region of the bone and this can be seen by the tensile stress arrows at the far end of the model. The blue and orange shapes on the borders of the model indicate the symmetry boundary conditions

Figure 18: (Left) The Kelvin-Voigt body form of the standard linear solid model that is capable of exhibiting stress relaxation and creep viscoelastic behaviors [50]. (Right) The 3D version of the rheological model where the spring and dashpot elements are replaced by shear and bulk moduli. .35 
Figure 19: The behavior of the tangent delta with respect to the viscosity is described by the best fit equation on the plot. With this equation, the value for the dashpot was determined by solving for the tangent delta found in the control ovine animals.

Figure 20: The test for convergence requires the inspection of a single node. The arrow points to the chosen node on the composite model. The zoomed in section reveals the stress distribution via the contour plotting tool in Abaqus.

Figure 21: The finer the mesh becomes, the more precise the analysis becomes. The data points appear to plateau at around the 3.25e-6 micron value which indicates that smaller mesh seed sizes are unnecessary as accuracy would not be increased significantly. 41

Figure 22: Graphical comparison between the experimental ovine data and the results from the Normal D-spacing variant of the composite model. The data has a coefficient of determination $\left(\mathrm{r}^{2}\right)$ of 0.918. 42

Figure 23: A plot of the viscoelastic properties for the DMA tests at varying frequencies. A dashpot value of $4 \mathrm{GPa}-\mathrm{s}$ for the FE model resulted in a less than desirable fit $\left(\mathrm{r}^{2}=0.746\right)$ to the experimental data. A dashpot value of $1.25 \mathrm{GPa}$-s for the model has a better fit $\left(\mathrm{r}^{2}=0.874\right)$ to the experimental data. .. 45

Figure 24: The viscoelastic data of the $15 \mathrm{~Hz}$ frequency tests are graphically represented here to reveal the relationship between tangent delta and mineral volume fraction.

Figure 25: The viscoelastic properties of the FE model and experimental ovine data are displayed as a function of frequency. The $1.25 \mathrm{GPa}-\mathrm{s}$ dashpot yielded $\mathrm{r}^{2}$ of 0.918 .

Table VI: Composite bone FE model data for the caudal side of the ovine bone using the $1 \mathrm{GPa}-\mathrm{s}$ dashpot. The target tangent delta originates from the experimental data.

Figure 26: The viscoelastic data of the $15 \mathrm{~Hz}$ frequency tests are graphically represented here to reveal the relationship between tangent delta and mineral volume fraction. .48 


\section{INTRODUCTION}

\subsection{Purpose}

Trying to understand the mechanical properties and overall characteristics of bone tissue is an ongoing challenge that should ultimately lead to advancements in health care involving metabolic bone diseases such as osteoporosis. About 10 million Americans have osteoporosis and another 34 million are at risk for developing the disease. In addition, it is very costly with approximately $\$ 19$ billion in related costs every year and a projected cost of $\$ 25.3$ billion by the year 2025 [5]. The unfortunate truth is that many individuals are diagnosed with osteoporosis after an osteoporotic related fracture has already occurred. This is because osteoporosis gradually develops with little to no symptoms and is often referred to as the "silent disease" $[5,52]$. The ideal solution is to avoid the initial fragility fracture altogether as this greatly increases the risk for additional osteoporotic fractures [77]. Understanding the exact mechanisms of osteoporosis through the use of computational models and animal testing can provide the insight needed to help create better diagnostic tools and therapeutic-preventative medicine. In order to deduce how osteoporosis affects bone tissue, a firm background of bone function, structure, and biomechanics must be covered.

\subsection{Bone}

\subsubsection{Function and Composition}

Bone is a remarkably optimized organ that provides structural support for the body, protects vital organs from impacts and falls, houses the blood cell producing bone marrow, acts as a mineral storage mostly for calcium and phosphorous, and supplies the framework necessary for motion [3, 4]. Bone is primarily known for its mechanical functions; however, this was not always the case as it was previously thought that bone's primary function involved the regulation of calcium [1]. Now, it is known that bone tissue is highly adaptable and one of the purposes of the cells comprising the tissue is to respond to mechanical stimuli and maintain its mechanical integrity through modeling and remodeling processes [1, 3]. The modeling and remodeling behaviors, as well as the cells that are involved, are discussed in a later section. 
The composition of bone matrix is just as important to understand as its many functions. The primary constituents of bone are collagen, water, and hydroxyapatite (HA). Collagen is a structural protein that is actually the most abundant protein found in animals. This protein also has many variations as there are 28 identified types of collagen [79]. The predominant form of collagen in bone tissue is known as type I and is also found in many other tissues such as tendon, dermis, and dentin. Collagen imparts flexibility and tensile strength to the bone whereas hydroxyapatite results in greater stiffness and compressive strength. The HA in bone is a version of calcium phosphate which forms small mineral crystals that are hundreds of angstroms long and wide and just tens of angstroms thick [36, 40, 47]. These crystals are embedded throughout the bone matrix. This process is described in detail in the following section. The water is either bound or free and also plays a major role in the mechanical properties of bone [57]. There are also small amounts of proteoglycans and other noncollagenous proteins that are not thought to have direct roles in the mechanical nature of bone. In fact, the precise functions of these noncollagenous proteins are fairly unknown, but it is thought that they play various roles in the mineralization process of the tissue [1]. 


\subsubsection{Complex Hierarchical Structure}

\subsubsection{Nano-scale}

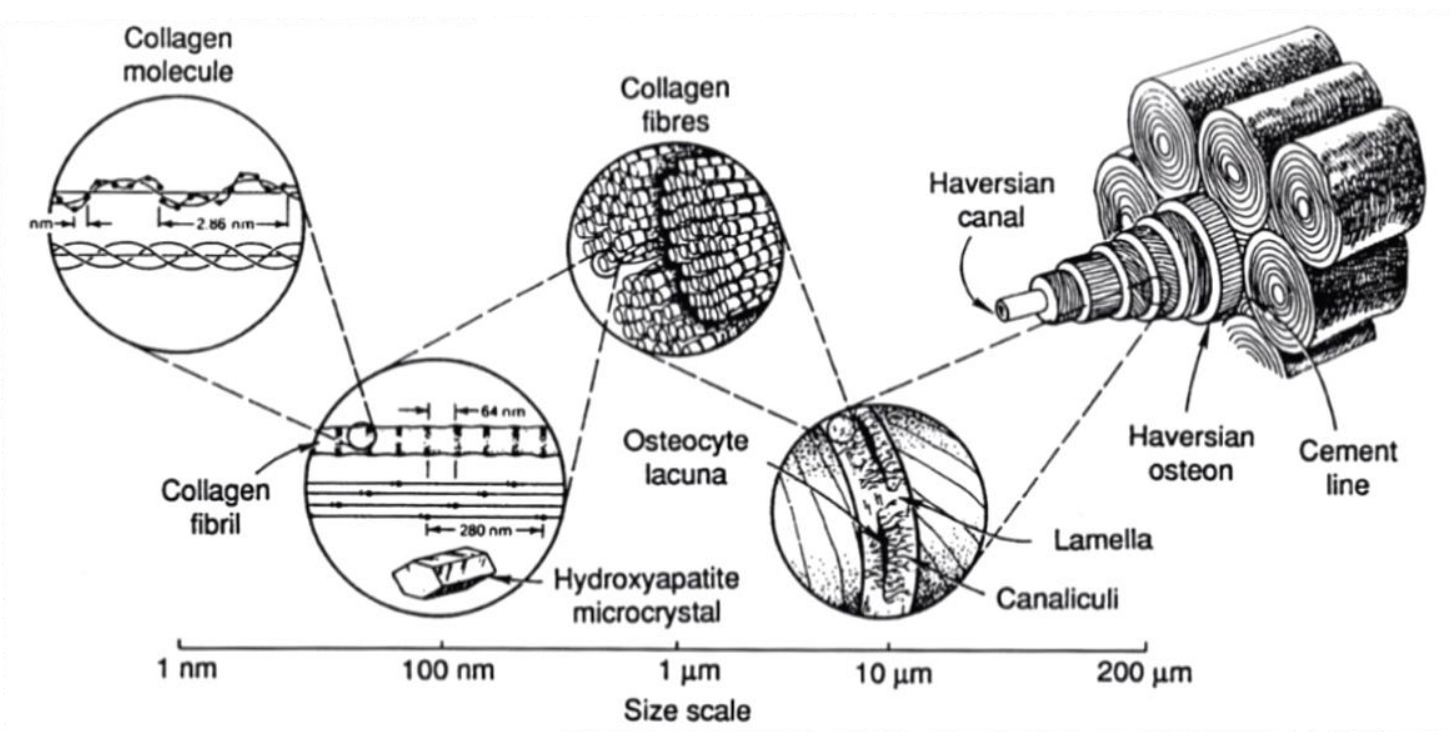

Figure 1: Hierarchical structure of bone starting from its basic composition [21]

Bone tissue has a highly complex hierarchical structure (Figure 1). At the lowest level, it can be thought of as an organized collagen matrix strengthened by the embedment of HA mineral crystals. The collagen fibrils are composed of tropocollagen molecules that are approximately 280 to $300 \mathrm{~nm}$ long and $1.5 \mathrm{~nm}$ in diameter [3]. The collagen network is originally organized in such a way that gap regions are created between the head and tail regions of the aligned molecules. The arrangement of these molecules is thought to be quite similar to the Hodge and Petruska model [39]. The spaces are initially filled with water prior to mineralization. The early stages of mineralization involve the filling of these gap regions with mineral crystals [1]. The crystals bind to the surrounding collagen molecules, displacing the water in the process. Additionally, neighboring columns of tropocollagen molecules are offset by approximately $67 \mathrm{~nm}$. This characteristic offset that collagen displays is known as D-spacing or D-period [2, 3]. While this value is the accepted D-spacing found in literature, there is much supporting evidence to show that it is actually a distribution of values throughout the tissue (Figure 2) $[8,57,58]$. 


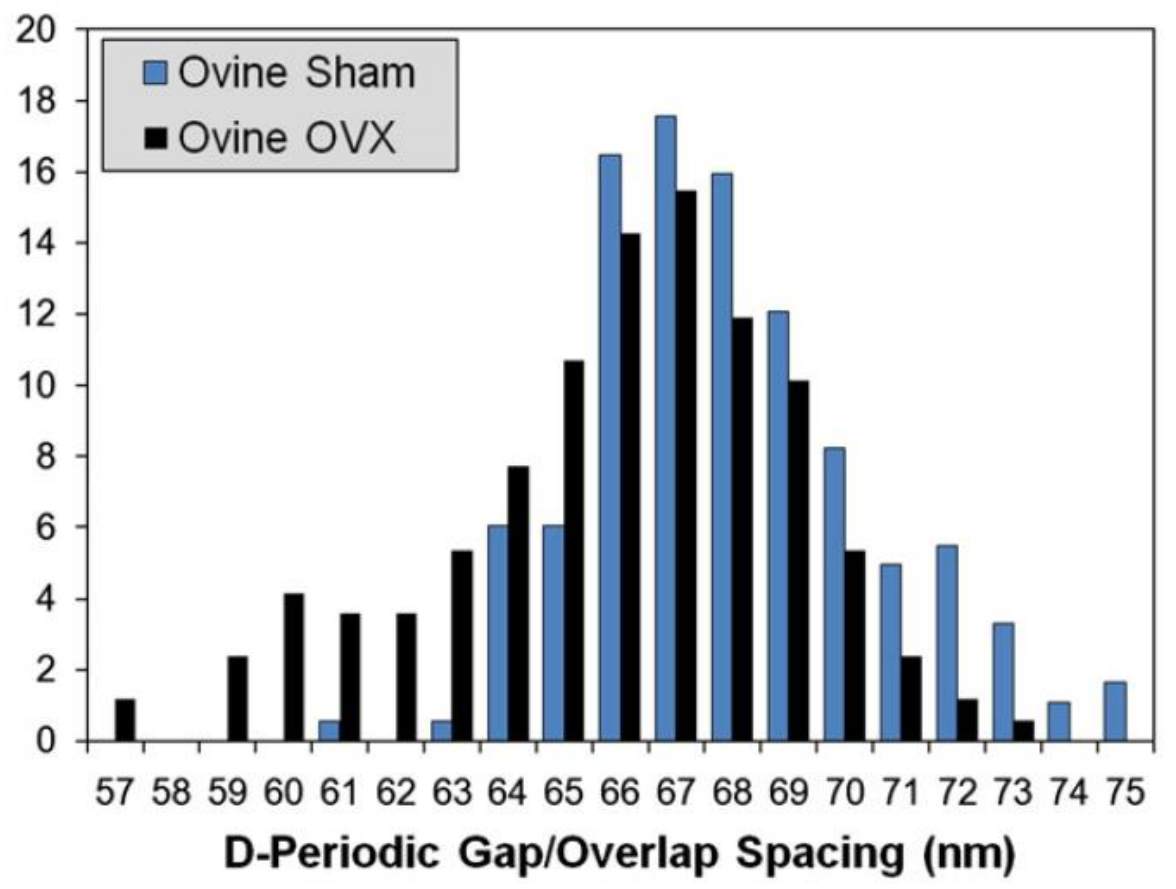

Figure 2: Distribution of D-spacing values found in ovine bone [8].

The arrangement of the tropocollagen molecules only describes bone tissue in the nanometer scale. The aligned molecules form fibrils which aggregate to create fibril bundles at the micron level. The individual molecules are covalently cross-linked intrafibrillarly as well as extrafibrillarly [59, 60]. These bundles are often referred to as fibers in tendon and dermis or lamellar sheets in bone. As stated before, the D-spacing in bone tissue is a distribution of values; however, the D-spacing within a particular fibril bundle appears to be constant. The distribution of $\mathrm{D}$-spacing is seen at the fibril bundle level and does not depend on tissue type or species [57]. It must also be stated that this behavior occurs with or without the presence of mineral [58]. 


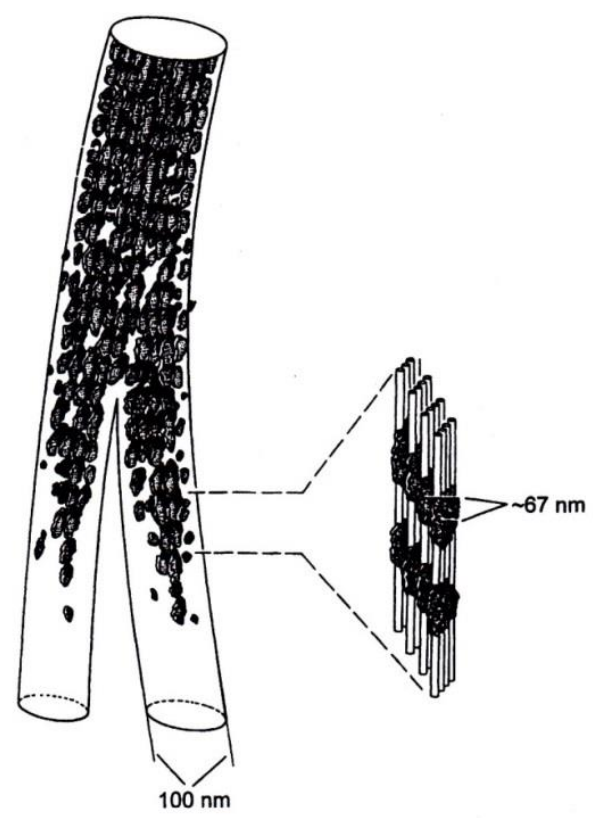

Figure 3: Image of mineralized turkey leg tendon revealing the progression of mineralization from bottom to top [54]

Figure 3 displays the distribution of the mineral crystals during the mineralization process in the avian tendon, one convenient model of the mineralization process. The D-spacing of the tropocollagen molecules is also shown with HA crystals located in the gap regions during the early stages of mineralization. The later stages of the mineralization process involve the embedment of mineral crystals between and within the collagen fibrils [40]. There is also evidence to support that more mineral is extrafibrillar as opposed to intrafibrillar [13]. At even higher levels in the bone hierarchy, the orientation of the collagen fibrils differs between each tissue type. 


\subsubsection{Micro-scale}

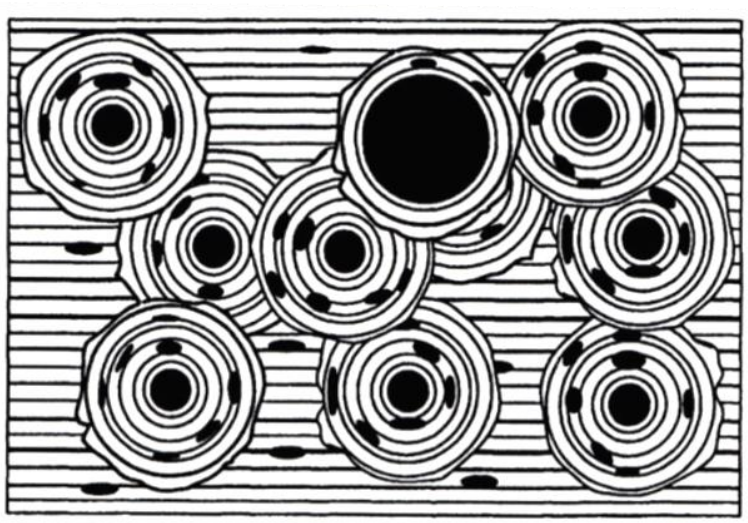

Figure 4: This image contains multiple Haversian systems in a field of lamellar bone. One of the osteons is incomplete and there are also various regions of osteon overlap [1].

There are four major types of bone tissue. The first type, called lamellar bone shown in Figure 4, consists of individual layers about 5 microns thick called lamellae. These lamellae contain parallel collagen fibers with HA crystals embedded throughout [2]. The orientation of neighboring layers changes, which results in a "twisted plywood" architecture [17]. This highly organized arrangement of lamellar bone accordingly possesses a slow rate of formation. More specifically, lamellar bone is laid down at a rate of less than one micron per day [53].

In contrast, woven bone can be laid down at a rate greater than 4 microns a day. This tissue is primarily seen in mammals during early stages of development and in calluses where fracture repair occurs. Also unlike lamellar bone, the structure of woven bone is highly unorganized. The collagen fibrils and mineral crystals that compose the material are randomly oriented [2]. Additionally, it is mechanically weaker but has the capacity to become more mineralized than lamellar bone [1]. This tissue is more or less temporary, especially in instances of fracture repair and during the rapid growth of larger animals [1, 2]. 


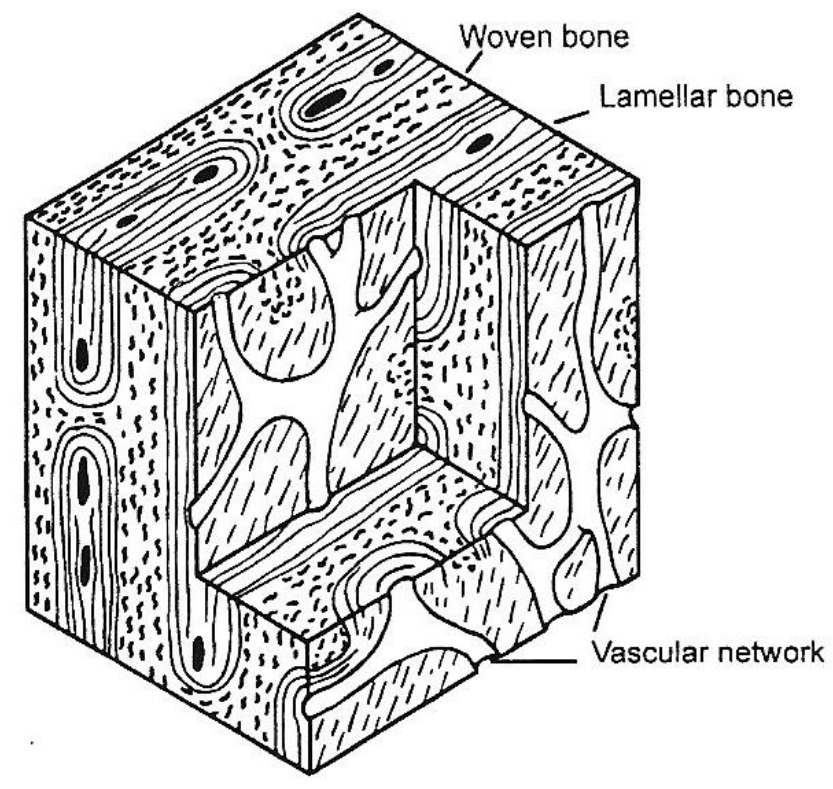

Figure 5: Diagram of plexiform bone [2]

Plexiform bone, also known as fibrolamellar and laminar bone, is the primary bone tissue type of large animals such as bovines and ovines. The plexiform tissue is a combination of woven and lamellar bone that facilitates the rapid growth of the skeletal system in prey species that must be able to effectively ambulate within hours of birth. Figure 5 displays how woven and lamellar bone are typically arranged in plexiform bone. The formation of this tissue starts with the quickly forming woven bone that is later incorporated with lamellar networks $[1,2]$.

The final type of bone tissue, called Haversian bone (named after Clopton Havers), is a form of lamellar bone. Haversian bone is formed via remodeling processes. The remodeling of the bone forms Haversian systems or secondary osteons roughly 200 microns in diameter and are composed of cylindrical lamellae that surround a cavity known as the Haversian canal (Figure 4). These Haversian canals provide pathways for blood vessels and nerves throughout the dense bone structures [1,2]. 


\subsubsection{Macro-scale}

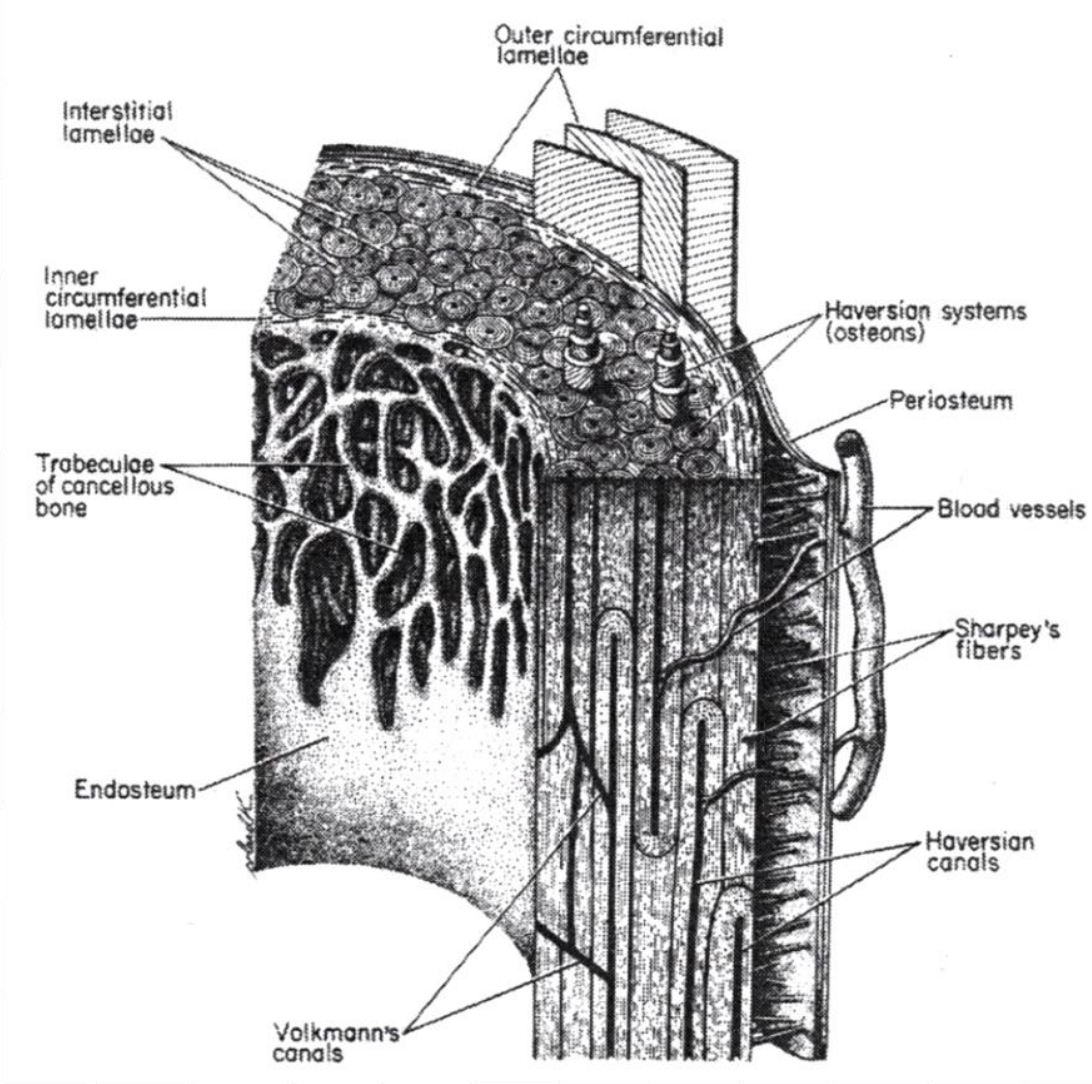

Figure 6: Structure of compact and trabecular bone [1]

At the highest hierarchical level, bone is categorized into two major classes based on the porosity of the tissue. If bone tissue has a porosity varying from 5 to 10 percent, it is considered to be compact bone. The porosity of compact bone is derived from Haversian canals, Volkmannn's canals, resorption cavities, lacunae from embedded cells, and canaliculi. In contrast, cancellous bone has relatively high porosity ranging from approximately 75 to 90 percent. Cancellous bone is also referred to as trabecular or spongy bone and often has a plate and strut configuration [1,2]. These plates and struts are known as trabeculae and their arrangement depends on their anatomical location and biomechanical function [3]. The shapes and thicknesses of the trabeculae may also be altered from disease states such as osteoporosis [3]. The spaces between the trabeculae are normally filled with red or yellow marrow depending on anatomic site and the age of the animal [4]. Red marrow is composed of blood vessels, nerves, and many 
other types of cells that produce the cells present in the blood [1], whereas yellow marrow contains adipose tissue and a few primitive blood cells [4]. Compact and cancellous bone are usually very easy to distinguish. Bone rarely has porosity between 50 and 15 percent, so the discrimination between these vastly different porous structures is relatively simple [2]. Figure 6 reveals the typical structural arrangement of compact and trabecular bone tissue of a long bone. Over the course of a mammal's lifetime, the skeletal system adapts and the porosity of the tissue fluctuates due to the organized activity of bone cells. These cells reshape, expand, remove, and replace bone tissue through processes known as modeling and remodeling.

\subsubsection{Modeling and Remodeling}

It's interesting and perhaps counterintuitive to think of bone as a living network of cells mostly because it is a highly calcified and stiff material. There are four primary cell types tasked with the responsibilities to grow the bone tissue and maintain its mechanical integrity. These cells are known as bone lining cells, osteoblasts, osteoclasts, and osteocytes. Bone lining cells are located on the surfaces of bone tissue including the inner (endosteum) and outer (periosteum) surfaces [1-3]. These cells direct the movement of ions between bone and the rest of the body [55] and are considered to be the quiescent form of osteoblasts. These osteoblasts are mononucleate cells that are responsible for bone formation as they initially lay down a collagenous matrix called osteoid composed of collagen, noncollagenous proteins, and water. The osteoid is later mineralized and the osteoblasts may also have a role in this process [1].

Osteoclasts serve the opposite function of osteoblasts. These cells are larger in size, multinucleated, and dissolve bone tissue by demineralizing it with acids and breaking down the collagen with enzymes. This bone resorption process occurs at a rate of tens of microns a day whereas osteoid formation is approximately one micron per day [1]. The fourth and final cell type called osteocytes are actually osteoblasts that have become embedded within the bone matrix in pores known as lacunae [1-3]. They have processes that extend into the bone matrix through canaliculi, which serve as communication 
and nutrient pathways [45]. Together with osteocytes, bone lining cells communicate with each other and are thought to initiate the remodeling process [1].

Modeling and remodeling involve the bone resorbing activities of the osteoclasts and bone forming actions of the osteoblasts; however, there are fundamental differences between the two. The modeling process does not require coupled actions from both cells; osteoblasts and osteoclasts act independently of one another. Modeling specifically refers to changes made to the bone shape, size, or both [1]. The more obvious cases of modeling occur during the early maturation of the skeletal system [2]. This includes adjustments made to long bone curvature, reductions/additions in long bone diameter, and the complex process of modeling flat bones such as the skull to accommodate the growing size of the brain. Upon reaching skeletal maturity, the rate of modeling is greatly reduced, but remodeling continues to occur throughout the organism's lifetime [1].

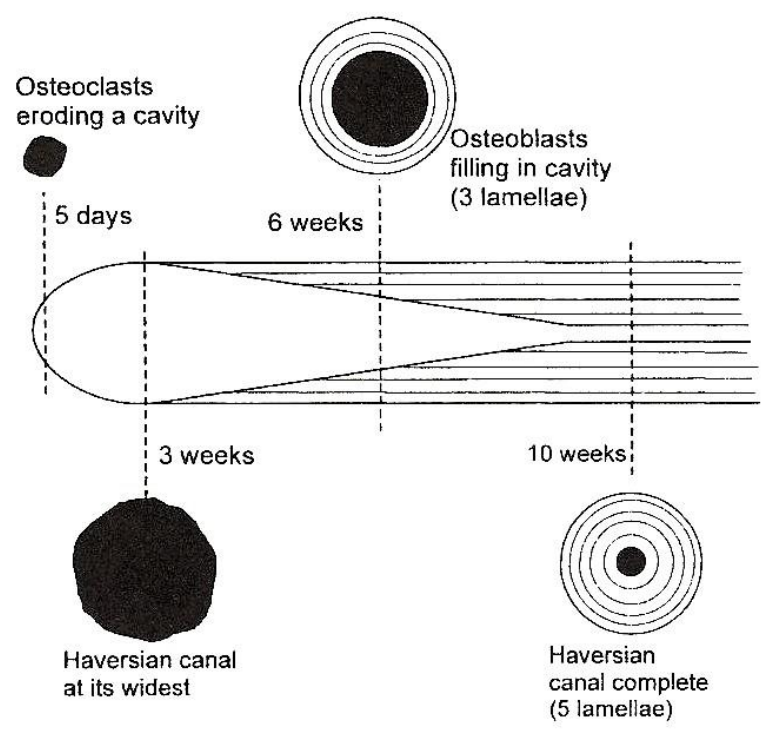

Figure 7: This diagram reveals the longitudinal cross-section of an osteon or Haversian system created by the remodeling activities of osteoblasts and osteoclasts [1].

Remodeling is essentially a bone replacement process; the amount of bone tissue is mostly maintained once remodeling has completed and the shape of the whole bone structure remains the same. Osteoclasts and osteoblasts remove and form bone in a sequential and coupled procedure. The organized 
action of these cells is often referred to as the basic multicellular unit or BMU [1, 2]. Osteoclasts first tunnel through bone tissue, leaving behind an uneven cylindrical space; this continues for roughly 300 microns at a rate of 40 microns a day. Osteoblasts then start the bone formation process by laying down layers of osteoid at about 1 micron a day. The whole process takes 2 to 4 months to complete and the resulting structure is an osteon composed of concentric lamellae and a central Haversian canal for blood vessels and nerves. Figure 7 is a longitudinal cross-section of an osteon and approximate timeline of its stages of development. The osteon is also surrounded by a region called the cement line, where the newly formed bone meets the old bone surface. The cement line contains small amounts of collagen and provides a mechanically inferior path to impede crack propagation and increase the ductility of bone [1].

\subsection{Skeletal Biomechanics}

\subsubsection{Bone as a Material}

Cancellous and compact bone have different mechanical properties primarily because of the differences between their structures. It has even been hypothesized that the intrinsic mechanical properties of the compact and trabecular bone are actually fairly similar; however, recent research has shown that cortical bone is indeed mechanically superior to cancellous with roughly 25 percent greater strength [61]. To understand why the mechanical differences are detectable, one would have to study the microarchitectural variations between the two. But for the purposes of this experiment, there will be an emphasis on the mechanical properties of compact rather than cancellous bone.

The collagen fiber orientation, varied hierarchical composite assembly, percent mineralization, porosity, and the dynamic nature of this living tissue all contribute to the orientation effects on the mechanical properties of bone. When measured, the number of elastic constants ranges from 2 to 21 [1]; this makes characterizing bone that much more difficult. Compact bone is typically considered an orthotropic material and much research has shown that it can be treated as a transversely isotropic material, particularly in Haversian bone [18]. Many researchers keep this in mind and tend to measure the mechanical properties of bone accordingly. An example of these measurements correspond to the values in Table I and the anisotropic nature of bone is clearly shown. These values are not meant to be the 
absolute elastic constants of compact bone, but rather a glimpse into the measured properties $[18,19]$. It's also important to understand that there are two primary methods to measuring these properties: ultrasound and mechanical testing [2]. Both mechanical and ultrasound measurements are displayed for comparison in Table I.

Table I: Elastic moduli, shear moduli, and Poisson's ratio for bone rewritten from Currey [2, 18, 19]. Units are in GPa for the moduli and the values are written in a specific ordermean/standard deviation (sample number) — where statistical data was available. Additionally, the subscripts 1,2, and 3 represent the radial, circumferential, and longitudinal directions with respect to the long axis of the bone.

\begin{tabular}{|c|c|c|c|c|c|c|c|}
\hline & \multicolumn{2}{|c|}{ Ashman et al. } & \multicolumn{5}{|c|}{ Reilly and Burstein } \\
\hline & \multirow{4}{*}{$\begin{array}{r}\text { Canine } \\
\mathrm{Ul}\end{array}$} & \multirow{4}{*}{$\begin{array}{l}\text { Human } \\
\text { Haversian } \\
\text { ound }\end{array}$} & \multirow{2}{*}{\multicolumn{2}{|c|}{ Human Haversian }} & \multicolumn{3}{|c|}{ Bovine } \\
\hline & & & & & \multicolumn{2}{|c|}{ Haversian } & \multirow[t]{2}{*}{ Fibrolamellar } \\
\hline & & & \multicolumn{2}{|c|}{ Mechanical } & \multicolumn{2}{|c|}{ Mechanical } & \\
\hline & & & Tension & Compression & Tension & Compression & Tension \\
\hline$E_{1}$ & 12.8 & 12.0 & 12.8 & 11.7 & 10.4 & 10.1 & 11.0 \\
\hline & & & $3.0(25)$ & $1.01(5)$ & $1.6(5)$ & $1.8(8)$ & $0.17(25)$ \\
\hline$E_{2}$ & 15.6 & 13.4 & 12.8 & 11.7 & 10.4 & 10.1 & 11.0 \\
\hline & & & $3.0(25)$ & $1.01(5)$ & $1.6(5)$ & $1.8(8)$ & $0.17(25)$ \\
\hline$E_{3}$ & 20.1 & 20.0 & 17.7 & 18.2 & 23.1 & 22.3 & 26.5 \\
\hline & & & $3.6(38)$ & $0.85(4)$ & $3.2(3)$ & $4.6(5)$ & $5.4(6)$ \\
\hline$G_{12}$ & 4.7 & 4.5 & \multicolumn{2}{|r|}{ - } & \multicolumn{2}{|r|}{ - } & - \\
\hline$G_{13}$ & 5.7 & 5.6 & \multicolumn{2}{|c|}{$\begin{array}{c}3.3 \\
0.42(10)\end{array}$} & \multicolumn{2}{|c|}{3.6} & $\begin{array}{c}5.1 \\
0.39(6)\end{array}$ \\
\hline$G_{23}$ & 6.7 & 6.2 & \multicolumn{2}{|c|}{3.3} & $0.25(22)$ & $\begin{array}{l}3.6 \\
5(22)\end{array}$ & $\begin{array}{c}5.1 \\
0.39(6)\end{array}$ \\
\hline$v_{12}$ & 0.28 & 0.38 & $\begin{array}{c}0.53 \\
0.25(24)\end{array}$ & $\begin{array}{c}0.63 \\
0.20(5)\end{array}$ & $\begin{array}{c}0.51 \\
0.24(5)\end{array}$ & $\begin{array}{c}0.51 \\
0.12(8)\end{array}$ & $\begin{array}{c}0.63 \\
0.23(6)\end{array}$ \\
\hline$v_{13}$ & 0.29 & 0.22 & $\begin{array}{c}0.41 \\
0.15(26)\end{array}$ & $\begin{array}{c}0.38 \\
0.15(4)\end{array}$ & $\begin{array}{c}0.29 \\
0.08(3)\end{array}$ & $\begin{array}{c}0.40 \\
0.21(5)\end{array}$ & $\begin{array}{c}0.41 \\
0.23(10)\end{array}$ \\
\hline$v_{23}$ & 0.26 & 0.24 & - & - & - & - & - \\
\hline$v_{21}$ & 0.37 & 0.42 & $\begin{array}{c}0.53 \\
0.25(24)\end{array}$ & $\begin{array}{c}0.63 \\
0.20(5)\end{array}$ & $\begin{array}{c}0.51 \\
0.11(5)\end{array}$ & $\begin{array}{c}0.51 \\
0.12(8)\end{array}$ & $\begin{array}{c}0.63 \\
0.23(6)\end{array}$ \\
\hline$v_{31}$ & 0.45 & 0.37 & $\begin{array}{c}0.41 \\
0.15(26)\end{array}$ & $\begin{array}{c}0.38 \\
0.15(4)\end{array}$ & $\begin{array}{c}0.29 \\
0.08(3)\end{array}$ & $\begin{array}{c}0.40 \\
0.21(5)\end{array}$ & $\begin{array}{c}0.41 \\
0.23(10)\end{array}$ \\
\hline$v_{32}$ & 0.34 & 0.35 & - & - & - & - & - \\
\hline
\end{tabular}

An interesting observation in the data arises from the differences between Haversian and fibrolamellar bone. While not drastically different, the moduli are indeed greater in fibrolamellar. This observation has been studied in the past and the initial conclusions were that an increase in porosity and 
decrease in mineralization, caused by the inclusion of the osteonal structures, resulted in the weakening of compact bone [23]. It's now known that the cement line surrounding the osteon also plays a role in the reduced mechanical properties [16] and increased ductility of bone tissue [1]. Further research indicates that the strength of the tissue decreases following osteonal remodeling for multiple modes of loading [18]. There has also been considerable research on the mechanical properties of individual osteons. This work classified the osteons based on collagen fiber orientation and percent mineralization. The results of this experimentation revealed that there are differing mechanical properties between the osteons themselves $[9,20]$.

The mechanical data described in this section scratches the surface of the experimentation done on compact bone. Only the elastic data was shown in order to introduce the mechanical characteristics of the tissue. In addition to the research that determined the elastic constants of the material, much research has focused on its mechanical failure including fracture mechanics, strength of materials, and damage formation. These concepts are all extremely important areas of study in the context of osteoporosis and other bone ailments. For instance, the strength of bone appropriately decreases considerably with the onset of osteoporosis [1]. Interestingly, bone is also viscoelastic and many of its mechanical properties are affected by the rate at which the material is loaded or strained. 


\subsubsection{Time Dependent Properties: Viscoelasticity}

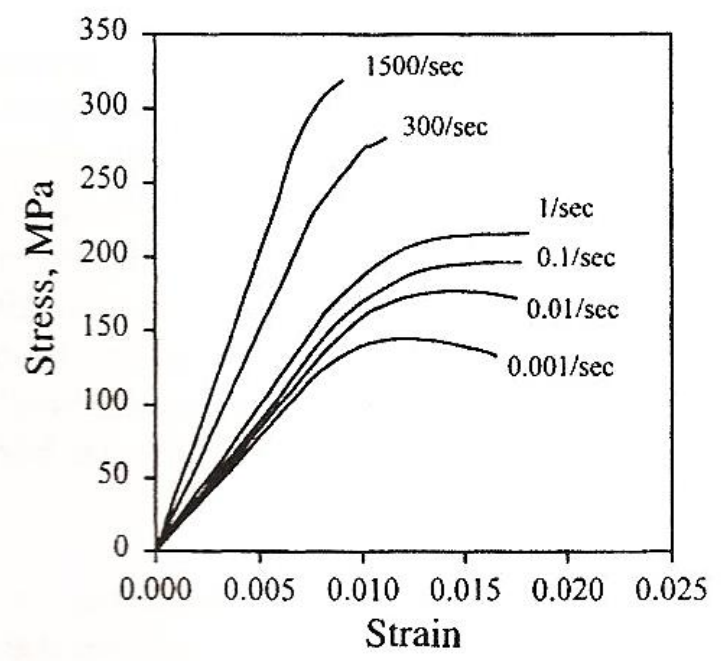

Figure 8: Bovine bone loaded in compression at different strain rates yield different stress-strain curves. The tissue becomes stronger, stiffer, and more brittle at higher strain rates [22].

Viscoelasticity is the phenomenon when a material exhibits both elastic and viscous properties as it deforms. Essentially, the measured elastic properties are not necessarily constant and various factors like loading or strain rate affect them. For instance, Figure 8 shown above reveals how bovine compact bone reacts to different strain rates. The elastic modulus, ultimate strength, and toughness are all affected by an adjustment to the strain rate [22]. Another experiment tested bovine femoral cortical bone in tension over a range of strain rates (including physiological rates) and found that the strength of the bone was proportionally related to the strain rate to the 0.07 power [25]. This tendency for compact bone to become stiffer, stronger, and more brittle at higher loading rates is a testament to its biomechanical function to maintain the mechanical integrity in events of trauma such as an impact during a fall.

Many living tissues are viscoelastic materials, but what makes bone viscoelastic? There has been considerable attention on determining the specific aspects of bone that lead to this behavior. Haversian bone, including the cement lines surrounding the osteons, may have an impact on these time dependent properties $[1,9]$. The presence of collagen in compact bone definitely contributes to this behavior as it was shown that biological materials lose their viscoelastic characteristics with a decrease in collagen 
content [33]. In addition, a dynamic mechanical analysis experiment on compact bone revealed that water plays a significant role as well [31]. Another series of research articles came to the conclusion that fluid in the vascular channels of compact bone does not significantly contribute to viscoelasticity but the interaction between the fluid and calcified matrix does [34]. More specifically, the interaction between water and the organic matrix, which is primarily composed of collagen, seems to impart the viscoelastic behavior [24]. So, water and collagen are important players in bone exhibiting time dependent properties. This is appropriate as other materials like tendon, ligament, and cartilage are also viscoelastic and are primarily composed of collagen and water $[1,3,4]$.

Viscoelastic materials are capable of undergoing many behaviors such as stress relaxation, creep, hysteresis, and more. Stress relaxation occurs when the material is strained to a specific value and held there. The material elastically responds with an instantaneous stress, but gradually the stress decreases over time. In creep, a constant stress is applied to the material. Once again there is an instantaneous response in the form of a strain, and a viscous increase in strain as the applied stress is held constant. Hysteresis describes the difference between the shapes of the loading and unloading curves $[1,2,4,10$, 44]. These three behaviors constitute some of the techniques used to observe and measure viscoelastic properties. A fourth technique known as the dynamic mechanical analysis (DMA) test applies a load that varies sinusoidally with time. The resulting strain curve expressed by the material is also sinusoidal, but there is a measureable lag between the curves as shown in Figure 9. This lag is known as delta and appears in the form of a phase shift $[10,33,44]$. 


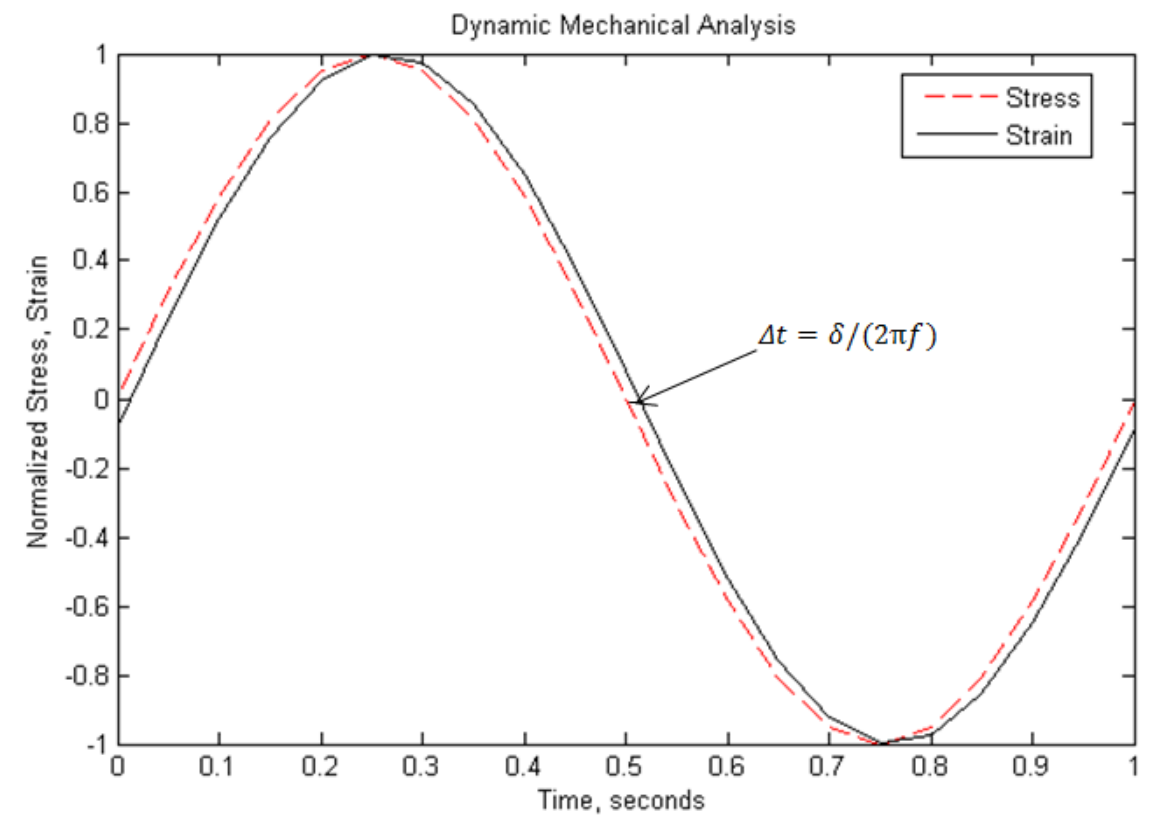

Figure 9: Stress and strain loading behaviors in a dynamic mechanical analysis test. The time difference, $\Delta \mathrm{t}$, between the sinusoids is related to delta as shown [44].

The stress and strain behaviors as functions of time take the form:

$$
\begin{gathered}
\sigma(t)=\sigma_{o} \sin (2 \pi f t) \\
\varepsilon(t)=\varepsilon_{o} \sin (2 \pi f t-\delta)
\end{gathered}
$$

where $f$ is frequency in $\mathrm{Hz}$ and $\delta$ is the phase shift in radians. The tangent of delta, appropriately called tangent delta or the loss tangent, describes how effectively a material can damp an oscillatory stress [42]. Typical values for tangent delta range from 0.1 to 1 for plastics and less than $10^{-3}$ for metals [46]. The convenience of running a DMA test as opposed to a stress relaxation or creep test is that it can be run in very short times (on the order of seconds or less) and multiple strain rates can be applied to the same specimen. Stress relaxation and creep do not accurately measure viscoelastic responses at such short times [10].

\subsubsection{Modeling the Mechanical Behavior of Bone Tissue}

Compact bone is a complex composite structure. It is essentially a multi-level composite starting with a mineralized collagen network, layers of lamellae with alternating orientations of collagen, and 
primary lamellar bone with osteons located throughout [1-3]. Many attempts have been made to simplify these structural complexities with various models in order to accurately represent bone for educational and research purposes. One of these early attempts simply modeled bone as a two phase composite of collagen and hydroxyapatite based on their volume fractions. The resulting models are known as the Voigt and Ruess models $[4,11]$. While these models provide a simple volume fraction approach, the accuracies of the representations are far from desirable with the Voigt and Ruess models merely providing upper and lower bounds for the elastic modulus of bone [1,2].

The two phase composite model does not account for any of the structural aspects in bone. Katz, who helped develop this early model, created another representation of compact bone with the inclusion of Haversian systems. By assuming that the osteons are hexagonally packed, the stiffness differs between the interstitial bone and Haversian systems, and the cement lines have relatively low stiffness he was able to develop a fairly accurate model of Haversian bone [16]. The mechanical properties of individual lamella were not accounted for in this model. Of course, there have been attempts to model bone at this level. Two particular examples approached the development of the lamellar model in a similar fashion: the Young's and shear moduli (for collagen and HA), mineral volume fraction, and geometry of the mineral crystals were all assumed. The primary difference between these representations arises from the characterization of the mineral crystals. The mineral was represented as rod-like structures in one model and plate-like in the other $[14,15]$. In addition to these mathematical models, finite element (FE) analysis programs like Abaqus can accurately represent biological materials. 


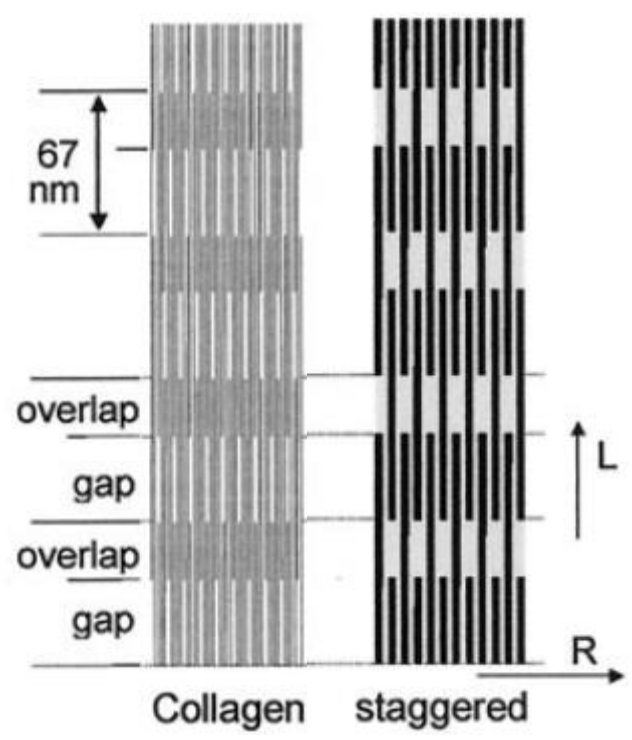

Figure 10: Comparison between various arrangements of collagen and HA. The Hodge-Petruska scheme is shown on the left and the staggered arrangement on the right. The characteristic D-spacing behavior results in overlap and gap regions that are maintained in the staggered array model [12].

Finite element analysis is a powerful tool that has been used to create computational models at multiple levels in the bone hierarchy. One such analysis characterized bone viscoelasticity based on experimental strain rate data and observed the effects of a press-fit femoral stem [37]. Another experiment modeled an individual transverse osteon and found that this structure is only effective in areas with dynamic compressive loads [41]. There have even been attempts to model bone at the molecular level. Even though the Hodge and Petruska model is the widely accepted molecular arrangement of collagen and HA [39], alternate models such as the staggered array model of Jager and Fratzel can be used to learn something about bone at the mineralized collagen fibril scale [12]. Figure 10 is a side by side comparison of the Hodge and Petruska and staggered array models. The analysis by Siegmund and co-workers computationally modeled the staggered array model and determined that an increased number of nonenzymatic collagen cross-links may reduce bone toughness [32].

\subsubsection{Modeling Viscoelasticity}

Viscoelastic materials add an extra level of difficulty in terms modeling their mechanical behavior. One of the simplest approaches to model these time dependent properties is to gather 
experimental data, such as strain rate data, and develop an experimental fit equation. The equation could be as simple as a relationship between the elastic modulus and strain rate. On the other hand, creating an accurate mathematical model can be extremely difficult as viscoelastic behaviors are complex and may depend upon temperature, loading type, rate of deformation, and more.

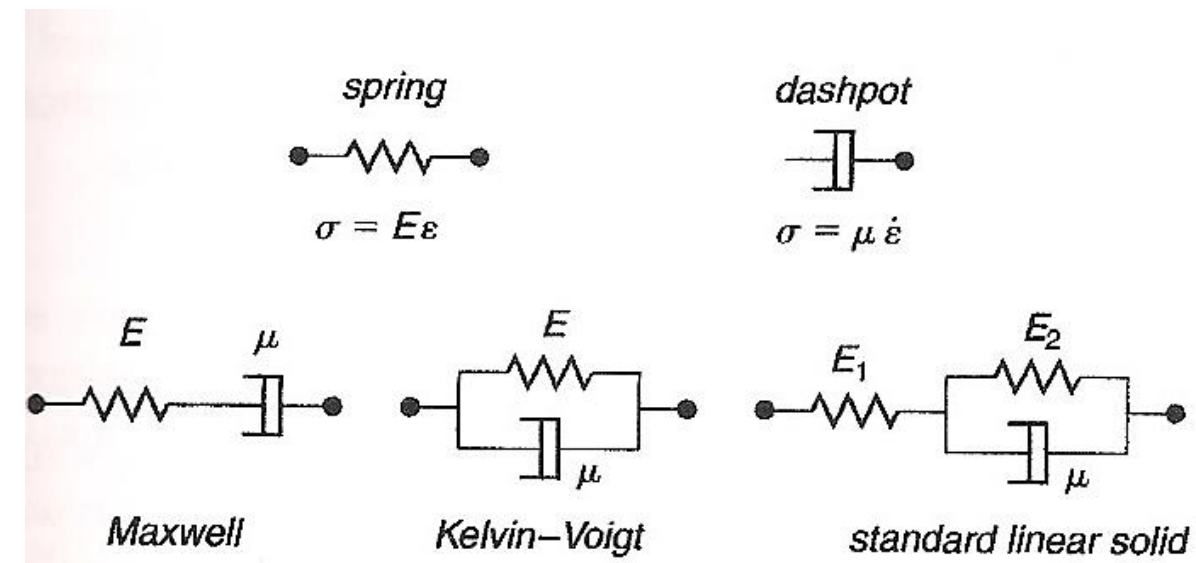

Figure 11: The spring and dashpot elements are shown with their governing equations. Three simple constructions including the Kelvin-Voigt body form of the standard linear solid are also displayed [4].

An alternate linear viscoelastic approach involves the use of springs and dashpots, or elastic and viscous elements to represent the material. Essentially, the springs and dashpots can be arranged in a network. Three examples of spring and dashpot networks are shown in Figure 11. Constitutive stressstrain equations can then be derived from the spring and dashpot network. The precise arrangement of the network, number of viscous and elastic elements, and element moduli and viscosities all affect the viscoelastic behaviors of the model $[3,4,44]$. These rheological models provide a manageable approach to viscoelastic representation with a much needed reduction in mathematical rigor; however, many biological materials react in a nonlinear way and require much more sophisticated modeling techniques [44]. 


\subsection{Osteoporosis}

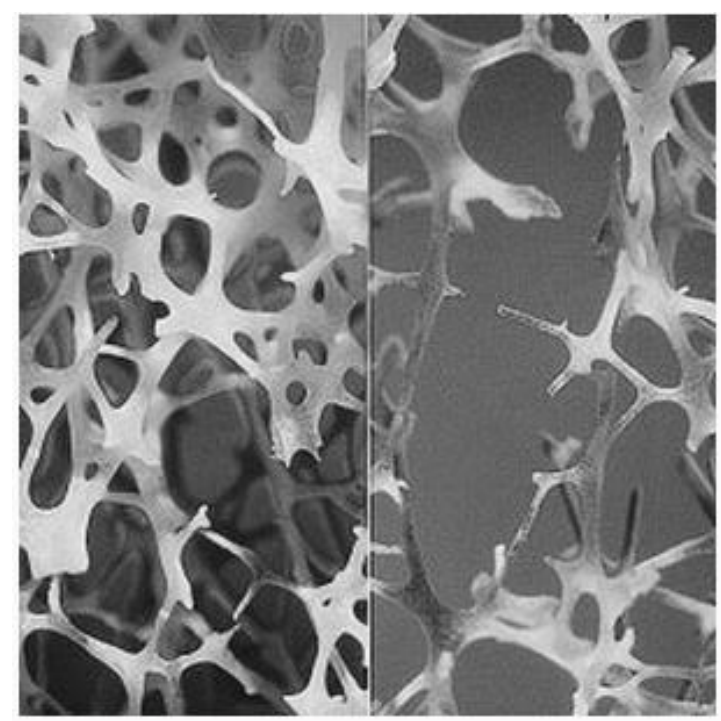

Figure 12: An image of normal bone on the left and osteoporotic bone on the right [52].

It is clearly known that the primary effects of osteoporosis involve the thinning of bone and loss of bone density (Figure 12); however, the exact mechanisms and consequences of this common bone disease are less understood. This bone ailment is typically detected with a bone mineral density (BMD) test. The measurements for BMD tests are normally taken with dual-energy X-ray absorptiometry (DXA). If an individual's BMD score is 2.5 standard deviations below the reference score from a population of young-healthy adults, they are diagnosed with osteoporosis [38]. The individuals with the greatest risk of osteoporosis include women who have experienced a drop in estrogen, usually around the time menopause occurs, and in people over the age of 50. There are additional risk factors that can be controlled including alcohol consumption, smoking, poor nutrition, vitamin D deficiency, and more [5, 52]. As many as one in five men and one in three women over 50 are at risk of having an osteoporotic related fracture [52].

The activity of the bone cells plays a prominent role in the development of osteoporosis as individuals with higher peak bone mass in early adulthood are less likely to get the disease. This is because the balance between bone formation and resorption shifts from positive to negative as age 
increases. This also means that individuals with osteopenia are more likely to develop osteoporosis. Osteopenia describes the situation where a person has lower than normal bone density, but not low enough to be considered osteoporosis [5]. The primary consequence of having osteoporosis comes in the form of increased fracture risk, even at relatively low impact speeds. The most common fractures occur in the hip, spine, and wrist; however, spinal and hip fractures are the most concerning as they can lead to intense back pain, deformities, immobility, and even death [52]. Even though men are also at risk for developing the disease, osteoporosis in women is of particular importance because of the increased risk factors associated with generally lower peak bone mass, longer life spans, and menopause.

\subsubsection{Post-menopausal Osteoporosis}

In menopause, the ovaries cease to function as endocrine organs. The body experiences a significant decrease in estrogen and progesterone resulting in an imbalance of bone metabolism [62, 64]. Specifically, the absence of estrogen increases the rate of remodeling where the amount of bone lost from resorption is greater than the newly created bone in formation [64]. The rate of bone loss is especially significant during the first two post-menopausal years [62]. It's also been shown that an induced early menopause through surgical intervention results in a higher incidence of osteoporotic fractures [65]. This hormonal deficiency directly results in the increased risk factor associated with post-menopausal women developing osteoporosis. The decline of estrogen is the primary contributor to the ensuing bone loss and the cellular mechanism describes how this occurs.

Estrogen depletion at the onset of menopause causes substantial amounts of bone loss via cytokine-driven increases in osteoclast formation. The cytokine process is not entirely understood; however, osteoclastogenic factors including RANKL, M-CSF, and tumor necrosis factor alpha (TNF- $\alpha$ ) are known to play significant roles in the postmenopausal loss of bone. Additional cytokines are involved and ultimately result in the increased remodeling behavior through specific mechanisms: an increase in the proliferation of osteoclasts, the longer lifespan of osteoclasts through the inhibition of apoptosis, and an increased capacity of mature osteoclasts to resorb bone [63]. 


\subsubsection{Current Treatment Options}

Arguably the best way to avoid osteoporosis is to prevent it from developing in the first place. Many of the risk factors discussed previously can be lowered or even avoided if the necessary precautions are taken. One of the best preventive methods involves attaining the highest peak bone mass possible. While peak bone mass is largely determined by genetic factors, this amount can be increased with plenty of exercise, appropriate calcium and vitamin D intake, and the avoidance of toxic habits [62]. This is an ideal approach but is limited to younger individuals as peak bone mass is reached in early adulthood. Oftentimes this prophylactic approach isn't enough, particularly in postmenopausal subjects, and can be further strengthened by the pharmacological agents used to treat the disease.

The immediate concern for osteoporotic patients is to prevent fractures from occurring. Increasing bone mass density (BMD) is an effective way to reduce fracture risk and ultimately treat osteoporosis. The treatments for the disease can be organized into two broad categories: anti-resorptive and anabolic. Anti-resorptive agents counteract or slow down the resorption process that occurs in remodeling. The primary goal of this treatment is to increase an individual's BMD. This is possible because the bone formation process is unaffected and the balance in bone metabolism shifts to promote bone growth. Anabolic agents stimulate bone formation rather than inhibit bone resorption in an effort to also increase BMD [5, 52].

Bisphosphonates such as alendronate and risedronate are the anti-resorptive type of osteoporosis treatment. They reduce bone resorption and bone loss either through the inhibition of the protein prenylation or the accumulation of toxic metabolites [66]. Some bisphosphonates are more tolerated than others; specifically, the adverse effects of alendronate are relatively mild with some transient gastrointestinal tract problems and some serious but uncommon events (esophagitis, gastric/duodenal ulceration/bleeding) [67]. Bisphosphonates are generally considered the gold standard for osteoporosis treatment; however, they also exhibit a unique behavior that is cause for concern. Bisphosphonates accumulate in the bone tissue and reside there for long periods of time. This behavior means that the 
remodeling processes may continue to be affected even after the treatment has ceased. In addition, bisphosphonates are capable of counteracting the effects of anabolic agents and this could prove to be problematic in the event that an individual chooses to seek alternative osteoporotic treatments. The long term effects of bisphosphonate accumulation are still not completely understood and the search for additional consequences continues today [66].

Alternative anti-resorptive agents include calcitonin, hormone replacement therapy (HRT), denosumab (a human monoclodal antibody raised against RANKL), and selective estrogen receptor modulators (SERM). Calcitonin is a polypeptide that is created by the thyroid and is available in subcutaneous injection or nasal spray form $[52,64]$. Despite having few adverse effects, its impact on BMD is relatively small [52]. HRT specifically refers to the replacement of estrogen for postmenopausal patients. While this option significantly decreases fracture risk, it would need to be prescribed for long periods of time [64] and the increased cancer and overall health risks generally outweigh the benefits. Denosumab slows osteoclastic function through the inhibition of the RANK-ligand. It is generally well tolerated with a slight increased risk of serious infection of the skin, urinary tract, and abdomen. A trial also showed that denosumab reduced fracture risk in postmenopausal women by 42 and 69 percent for hip and vertebral fractures respectively [68]. SERM agents such as tamoxifen and raloxifene inhibit resorption by interacting with estrogen receptors as either agonists or antagonists. Raloxifene in particular reduced the risk of vertebral fracture up to 49 percent but is also associated with increased stroke and venous thromboembolism risk [69].

Anti-resorptive agents are usually considered first to treat osteoporosis, but anabolic agents like teriparatide are also available. Teriparatide is the 1-34 N-terminal fragment of human parathyroid hormone. It promotes bone formation by interacting with cell-surface receptors found on osteoblasts and renal tubular cells. The most common adverse effects include limb pain, nausea, headaches, and dizziness and other more serious effects were uncommon [70]. Teriparatide strongly increases BMD in trabecular compartments which is why the greatest improvements are seen in the lumbar vertebrae [52]. This 
treatment has a high cost so it is recommended for individuals with high fracture risk or for those who require an alternative to anti-resorptive agents [70]. In addition to taking either anti-resorptive or anabolic agents individually, there has also been considerable research combining multiple treatments for increased positive outcomes $[66,70]$.

\subsubsection{Animal Models for Osteoporosis}

Research and experimentation utilizing animal models are necessary for the development and efficacy of medical devices and pharmacological agents alike. The same is true for research that attempt to understand the mechanisms behind diseases and complications such as osteoporosis. The obvious drawback of using animal models is that the exact situation that would occur in a human being could not be attained due to the biological differences between the species; however, even if only a few aspects of the disease or the biology are mimicked, the experimentation can provide invaluable insight into the disease and its mechanisms [71]. When searching for the best suited animal model for a particular study, the following considerations must be accounted for: the biological similarities and differences, transferability of information, genetic relation, cost and availability, ease of management and handling, and ethical implications [74].

For osteoporosis in particular, small animal models — specifically the rat-have been used extensively for studying bisphosphonates and other treatments and their effects on bone metabolism. This is because rodents are easy to handle, relatively inexpensive, and less time consuming $[71,73]$. Some of the biggest issues with rodents in osteoporosis research are that they are small in size which limits their use in orthopedic analyses, the trabecular remodeling differs from humans, and the osteonal structures that do appear are rare and scattered [72, 73]. Many large animal models including dogs, cats, pigs, sheep, and non-human primates have been utilized for osteoporosis research in an effort to avoid some of the small animal model limitations [73] and also incorporate mechanical testing on larger bone structures [8, 42, 43]. The ewe, or female sheep (Ovis aries), in particular has become a promising large animal model for postmenopausal osteoporosis. 


\subsubsection{Large Animal Model: The Ewe}

Ewes are docile in nature and very easy to handle. The costs to acquire and maintain these animals are low and the ethical concerns for sheep are not as sensitive as their large animal model counterparts [71, 73]. They're also genetically closer to humans than are rodents [73]. The similar bone size and structure of adult sheep provides even more incentive to use ewes in osteoporosis research [71, $72,73]$. The size of the bones also makes ewes a desirable candidate for fracture healing studies, another significant aspect of the osteoporotic condition [71]. Unlike human bone, the primary tissue of young sheep consists of plexiform bone which is later replaced with Haversian as they age (Figure 13) [71, 72, 76]. In addition, $\mathrm{BMD}$ and bone mineral content (BMC) are much greater in the ovine species than in humans. As far as osteoporosis research involving drug therapy goes, the differing gastrointestinal system and phosphorous metabolism is a major disadvantage [71]. Other significant biological aspects of ewes are especially important in postmenopausal osteoporosis research.
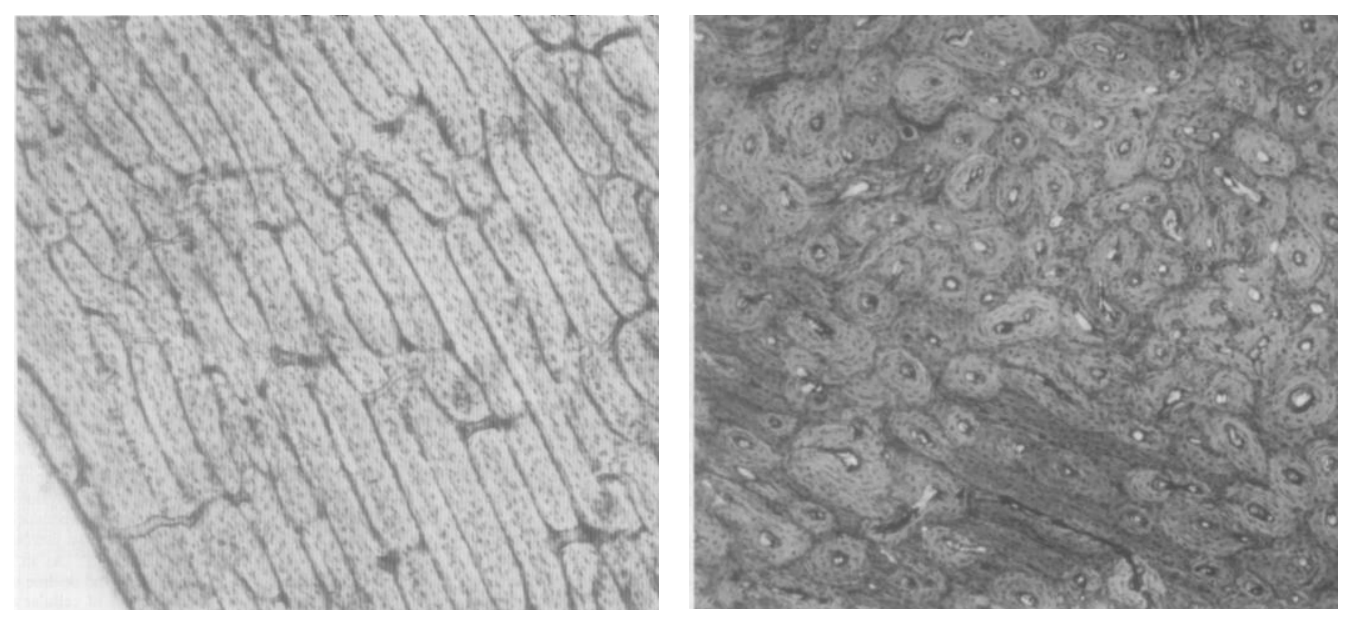

Figure 13: The images shown here are histological cross-sections of ewe bone. (Left) The bone is primarily composed of plexiform bone at a young age. The ewe was 3 years of age. (Right) Heavy remodeling throughout the sheep's lifetime yields Haversian bone that is very similar to adult human cortical tissue [76].

For the most part, ewes from Northern European breeds (the predominant varieties seen in North American production) have seasonal estrous cycles with high estrogen levels in autumn and winter and low in the spring and summer. The Merino sheep, a Spanish breed, is a notable exception with an almost 
continuous estrous cycle $[71,73]$. This means that research should account for the seasonal variability where applicable. Humans not only have a continuous estrous cycle, but also naturally undergo menopause. This behavior is typically only seen in other primates like Old World monkeys and great apes [71]. So, the use of ewes as a postmenopausal osteoporosis animal model requires the removal of the ovaries. This surgically induced menopause, or ovariectomy (OVX), is often coupled with alternative techniques such as dietary manipulation in order to facilitate the rapid bone loss effects normally seen in postmenopausal women [6]. One possible complication with the use of ewes is that the importance of estrogen and its impact on bone turnover is not as substantial as that seen in women. Many studies have not caused a considerable enough level of bone loss for the specimens to be considered osteoporotic, which may also be due in part to the increased BMD and BMC in ovines [71]. A large number of postmenopausal osteoporosis studies explicitly state that the ewes involved have become osteopenic [6, 42, 43, 71, 73]. Nevertheless, important findings have been made that may have significant implications in the osteoporotic condition. 


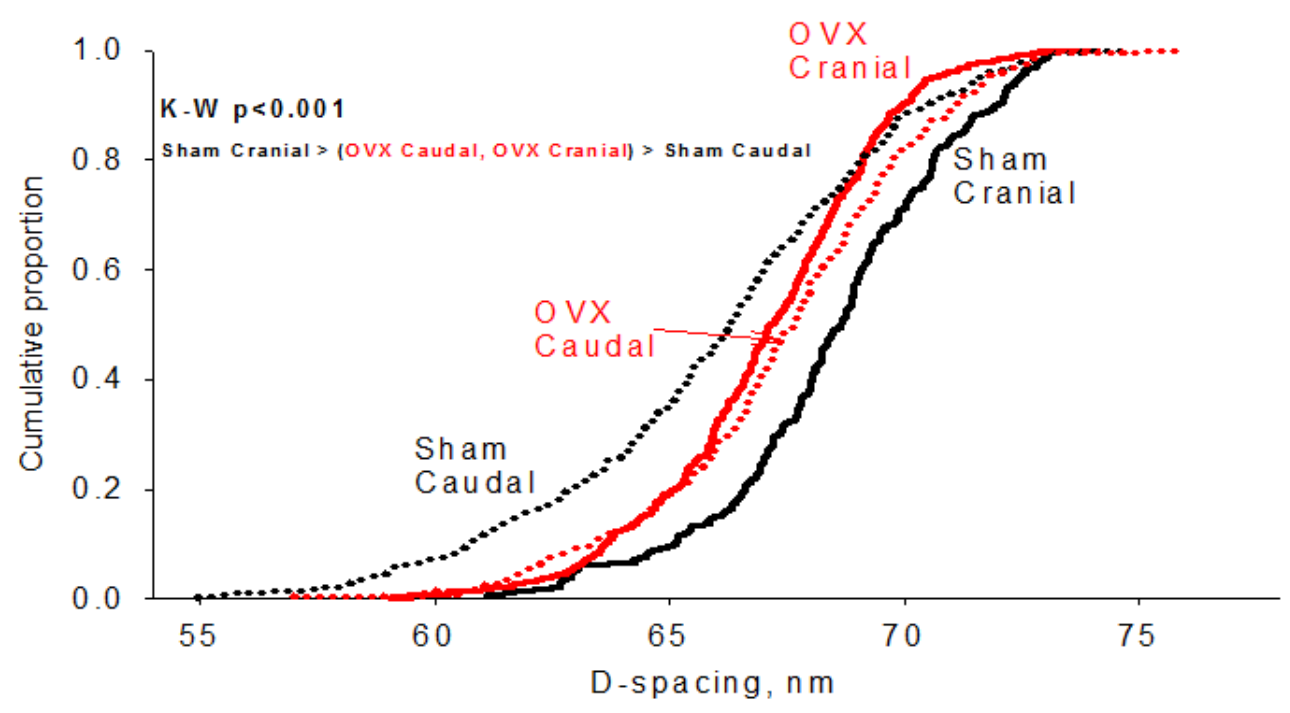

Figure 14: Collagen I fibril D-spacing as a function of anatomic site and treatment. The results seem to indicate a more homogeneous distribution of D-spacing in the cortical bone tissue caused by the ovariectomy [7].

Since the risk of osteoporosis increases greatly with the onset of menopause, many experiments have been proposed and completed to understand the exact mechanisms that take place during this stage of hormonal adjustment. There is evidence to suggest that estrogen depletion associated with menopause affects the mechanical properties and structure of the bone tissue prior to significant amounts of bone loss $[6,7,8,42,43]$. One particular postmenopausal ovine experiment found that the D-spacing distribution found in bone is altered by induced estrogen depletion (Figure 2). More specifically, the long term estrogen depletion suggests an overall decrease in D-spacing [57]. Another study revealed an interesting behavior that is dependent on anatomic site: the D-spacing either increases or decreases resulting in a more homogeneous material (Figure 14) [7]. Additionally, the changes that do occur in the bone matrix may occur independently of remodeling. It was found that the viscoelastic properties of cortical bone tissue were altered without significant changes in mineral density or microarchitecture. More importantly, these types of effects are not detectable by standard BMD tests for osteoporosis [42]. If the viscoelastic properties of cortical bone are changing ahead of the osteoclast mediated bone resorption seen in 
osteoporosis, the tissue strength and toughness may be compromised not only by the loss of bone tissue, but also by these structural and mechanical changes [43].

\subsection{Study Objectives}

The purpose of this FE analysis is to create a composite model of bone and simulate the three point bending test completed on ovine bone samples $[42,43]$ in order to determine whether the change in D-spacing is sufficient to describe the viscoelastic changes that occurred in the OVX ovine specimens. It's also valuable to understand if D-spacing impacts the viscoelastic behavior of the model at all. The logic behind examining the effects D-spacing has on bone mechanical properties is that collagen plays a pivotal role in bone viscoelasticity [24] and any alterations to the biochemistry of collagen through ageing

effects [28, 29], or other means [26, 27], significantly alters the mechanical integrity of the whole bone. In order to appropriately explore the effects that D-spacing imparts on the mechanical properties of bone, it's imperative to develop multiple model variants that account for the possible mechanisms that result in altered D-spacing values. In addition, it is desirable to determine which model variants exhibit the same behavior seen in the experimental OVX ovine data and the possible implications of such knowledge. Since D-spacing is a structural aspect of collagen that occurs at the nano scale, a simplified composite model is necessary to achieve these goals. 


\section{METHODS}

\subsection{Experimental Data: Specimen Preparation}

The composite bone model detailed in this section is based on ovine bone that had undergone three point bending experimentation to analyze the viscoelastic properties. Under local ACUC approval, 6 Warhill ewes were anesthetized and either ovariectomized or underwent a sham surgery. The animals were sacrificed after 3 years and the radius and ulna bones (the radius and ulna tend to fuse together in the ovine species) were harvested and stored at $-20{ }^{\circ} \mathrm{C}$. The whole bone was divided into six separate anatomic sectors (Figure 15); however, only the cranial and caudal variability in the data were accounted for in this FE analysis. Sections of $1.75 \mathrm{~mm}$ x $1.75 \mathrm{~mm}$ x $19 \mathrm{~mm}$ beams were then machined from each of these sectors for a total of 24 specimens. These beams were then stored in a $0.9 \%$ saline solution at -20

${ }^{\circ} \mathrm{C}$. In preparation for the DMA tests, the beams were thawed and placed in a $0.9 \%$ saline solution at 37 ${ }^{\circ} \mathrm{C}$. The viscoelastic data were measured with a dynamic mechanical analyzer (DMA 7e, Perkin-Elmer). Refer to Appendix A to view a table of the measured data from the experimentation.

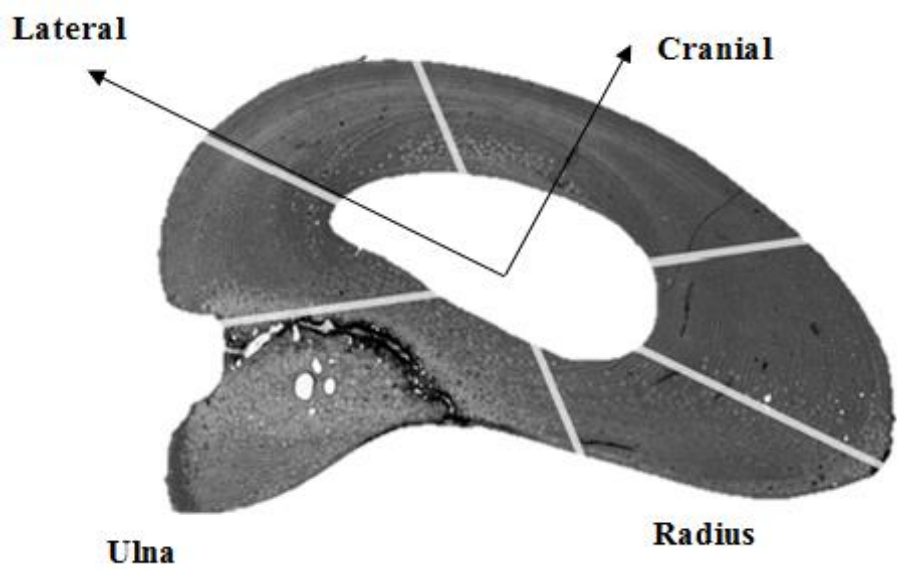

Figure 15: Cross-section of the radius and ulna bone from the ewes. The anatomical sectors are separated by gray lines.

Based on the geometry of the beams, the applied forces, and the dimensions of the three point bending apparatus, the associated stresses were determined in the compressive and tensile regions of the bone. The beam sections were considered a homogeneous material to estimate the stresses which were then applied to the computational model. Refer to Appendix B for detailed information on the bending 
stress calculations. The primary goal of the model is to determine if alterations to the collagen D-spacing that is characteristic of mineralized collagen fibrils affects the viscoelastic properties of the material. With this in mind, a model that accounts for the nano scale D-spacing behavior is required to observe the effects on the time dependent properties of the structure.

\subsection{Model Development}

In the interest of reducing computational time, it was decided that the model be simplified to a 2 dimensional analysis; however, because of the complicated structural nature of compact bone, the arrangement of the composite material would have to be determined. One such model, the staggered array model, simplifies the arrangement of collagen and hydroxyapatite in compact bone [12]. This simpleperiodic model was utilized in order to decrease the model size and analyze bone in a 2 dimensional plane strain analysis similar to another experiment that observed the effects of collagen cross-linking in mineralized collagen tissues [32]. Figure 16 shows the descending order of the geometric simplification.

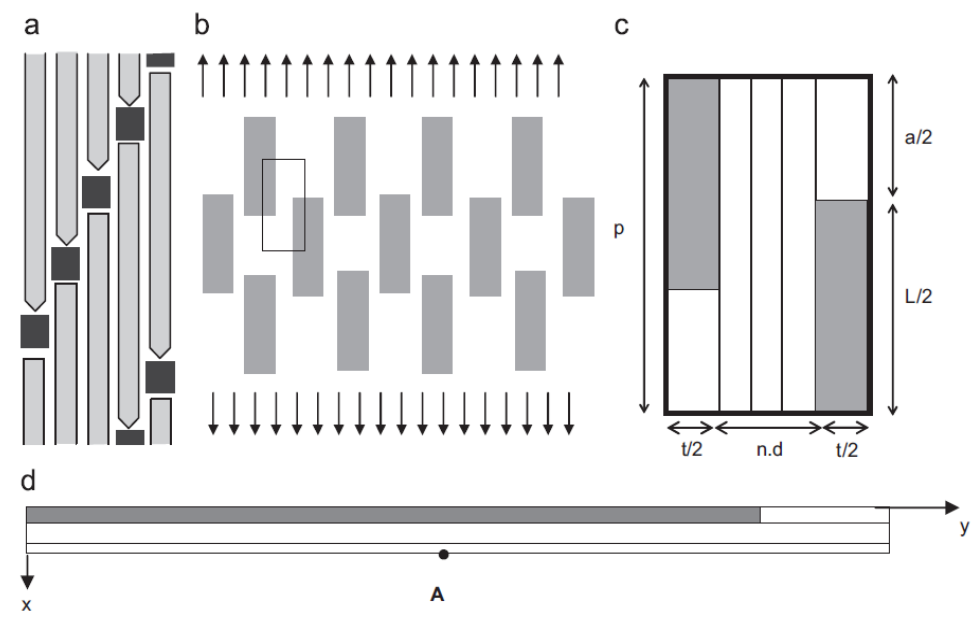

Figure 16: a) The arrangement of collagen (light grey) in between HA crystals (black) can be seen as well as the D-spacing behavior exhibited by mineralized collagen fibrils. b) The periodic arrangement of the collagen (white) and HA (grey) are shown. c) This image shows a single cell containing regions of HA and collagen interacting with one another. ' $P$ ' represents the periodic element length $(67 \mathrm{~nm})$ [12, 32]; ' $t$ ' represents the HA thickness $(2.5 \mathrm{~nm})$ [36]; ' $L$ ' represents the HA crystal length $(112.56 \mathrm{~nm})$ [36, 40, 47]; ' $a$ ' represents the distance between the mineral $(21.44 \mathrm{~nm})$; ' $n$.d' represents three collagen triple helices between the mineral $\left(3^{*} 1.5 \mathrm{~nm}=4.5 \mathrm{~nm}\right)$. d) The final model where HA is in dark grey and the white represents the collagen.

The staggered array model provides many benefits: the simplified bone geometry could be utilized for each analysis, the size of the model can be drastically reduced due to its periodic nature, and it 
includes the overlap and gap regions caused by the collagen D-spacing behavior. It's important to note that the staggered array model is based on six different variables: mineral volume fraction $\left(\mathrm{V}_{\mathrm{V}}{ }^{\mathrm{m}}\right)$, collagen length (a), collagen width (d), mineral length (L), mineral width (t), and periodic unit length $(\mathrm{P})[12,32]$. Each of the variables, with the exception of mineral volume fraction, and their associated values are described in detail in Figure $16 \mathrm{c}$. These specific dimensions were configured with the assumptions that the mineral volume fraction is 0.3 [35], the mineral plate width is $2.5 \mathrm{~nm}$ [36], the number of collagen domains between the mineral crystals is 3 , the width of collagen is $1.5 \mathrm{~nm}$ [3], and the periodic unit length is $67 \mathrm{~nm}[2,3]$. The relationship between the collagen fibers and mineral crystals is not an exact situation you would observe in mineralized collagen fibrils; however, the characteristic D-spacing behavior is maintained in the staggered array model. This behavior is directly related to the periodic unit length of the model. The following equations characterize the relationships between all the variables in the staggered array model:

$$
\begin{gathered}
P=\frac{(L+a)}{2} \\
V_{v}^{m}=\frac{L t}{(L+a)(b+t)}
\end{gathered}
$$

The first equation reveals the relationship between the periodic unit length $(\mathrm{P})$, the mineral crystal or HA length (L), and the collagen length (a). Equation (2) relates the mineral volume fraction $\left(\mathrm{V}_{\mathrm{v}}{ }^{\mathrm{m}}\right)$ to the mineral length, collagen length, mineral thickness ( $t$ ), and the collagen width between the mineral crystals $(\mathrm{b}=$ n.d, see Figure $16 \mathrm{c})$.

Table II: Elastic material properties of collagen and hydroxyapatite [1, 2, 4].

\begin{tabular}{|l|l|l|}
\hline \multicolumn{1}{|c|}{ Material } & \multicolumn{1}{|c|}{ Elastic Modulus (GPa) } & \multicolumn{1}{c|}{ Poisson's ratio } \\
\hline Hydroxyapatite & 100 & 0.28 \\
\hline Collagen & 2 & 0.2 \\
\hline
\end{tabular}

One important concept to understand is that Abaqus doesn't directly handle geometric lengths in the nano scale, so the composite model dimensions were entered in microns (i.e. the $67 \mathrm{~nm}$ periodic unit length would be written as $67 \mathrm{E}-3 \mu \mathrm{m})$. The elastic modulus and applied stresses were adjusted 
accordingly. In other words, a hydroxyapatite elastic modulus of $100 \mathrm{GPa}$ was entered as $0.1 \mathrm{E} 12 \mathrm{~Pa}$. Table II shows the values for elastic modulus and Poisson's ratio for collagen and HA if they are both treated as elastic materials. The model was originally tested with these material properties in order to evaluate the staggered array model. The collagen material properties were later altered to account for the viscoelastic behavior normally seen in compact bone. The details of the viscoelastic material definition are discussed in the following section.

Because of the periodic nature of the collagen-HA composite, symmetric boundary conditions were applied at $\mathrm{y}=0, \mathrm{y}=3.5 \mathrm{~nm}$, and $\mathrm{x}=0$ (according to the global coordinate system). Figure 17 shows the origin of the coordinate axis with respect to the model and where the symmetric boundary conditions are located. For additional clarity, the HA region is highlighted in red. Also, the entire model is a single part that is partitioned in order to apply the material properties to the appropriate sections. The fact that the entire model is a single part in Abaqus implies that the HA and the collagen are perfectly bonded. This may be appropriate to assume since the analysis is purposely in sub-yield testing conditions in order to coincide with the sub-yield testing procedure in the three point bending tests previously described. Since the ovine experiments were DMA tests with sinusoidal loading conditions, the periodic amplitude feature in Abaqus was applied to the model to mimic the real world situation as much as possible. Figure $17 \mathrm{c}$ displays arrows to the far right of the model which indicates the direction and location of the sinusoidal loading conditions. In addition, the loading conditions in the ovine testing were implemented at increasing frequencies $[42,43]$. The $1,3,9$, and $15 \mathrm{~Hz}$ frequencies were simulated on the composite bone model for comparison to the ovine data. An in-depth look at adjusting loading and boundary conditions through the input file is covered in Appendix C. 
a)

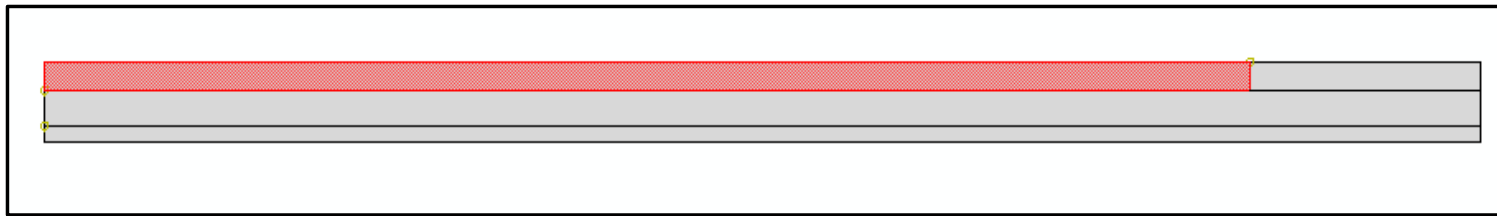

b)
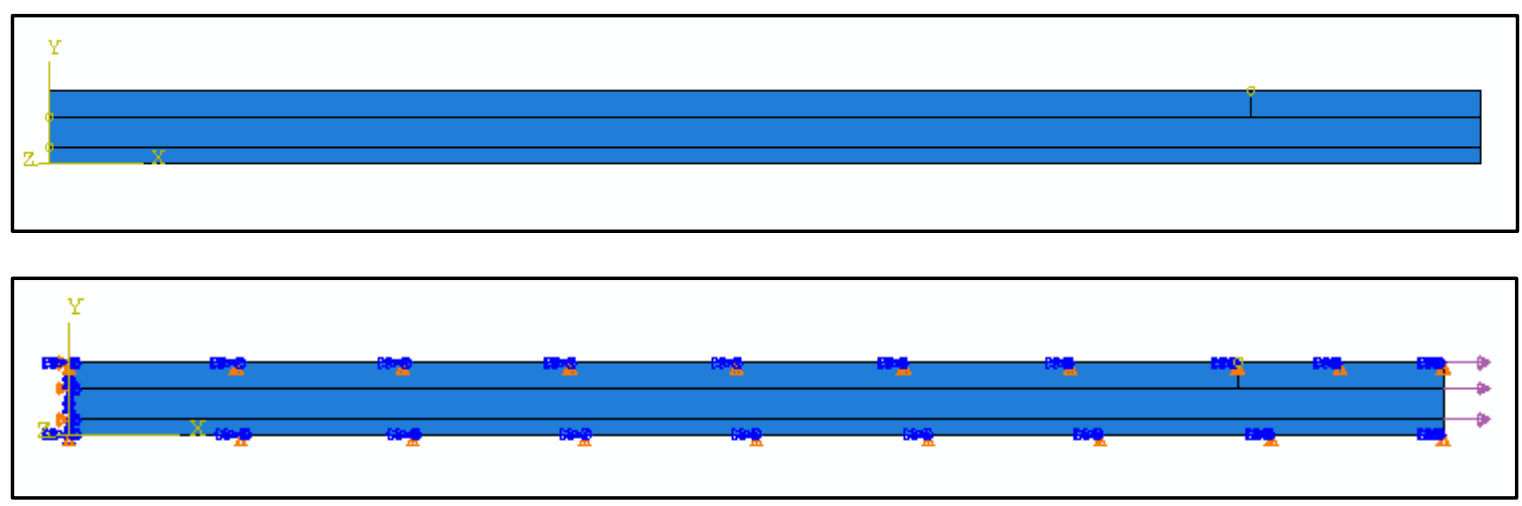

Figure 17: (a) Normal D-spacing model with red highlighted region indicates the mineral crystal. The gray regions represent the collagen domains. (b) The coordinate axis is shown in yellow at the far end of the model. (c) The boundary conditions and loads can be seen on the composite bone model. This model is on the tensile region of the bone and this can be seen by the tensile stress arrows at the far end of the

model. The blue and orange shapes on the borders of the model indicate the symmetry boundary conditions

The specific dimensions of the staggered array model that were just described coincide with the primary model designated as the "normal D-spacing model." In order to analyze the effects that Dspacing has on the viscoelastic properties of the composite material, additional models were produced to modify the D-spacing. High D-spacing and low D-spacing models have a periodic unit length of $73 \mathrm{~nm}$ and $61 \mathrm{~nm}$ respectively. These numbers were determined by observing a distribution of D-spacing measurements from ovine samples [8]. As stated beforehand, the dimensions of the staggered array model are characterized by six variables. With the intention of altering the D-spacing or periodic unit length, other variables must change to accommodate these deviations. The first models retained the mineral volume fraction of the control model. Previous studies have shown that the viscoelastic properties of bone change without significant alterations to the percent mineralization [51]. Alternatively, additional models were produced to alter the lengths of either the collagen or mineral crystals. This would cause the mineral volume fraction of the model to change as well. The logic behind these models is that most of the mineral 
crystals are actually located outside of the collagen fibrils as opposed to within [13]. The mineral located within the collagen fibrils create the characteristic D-spacing behavior [2, 40]. So, small changes to the mineral volume fraction of the model would not significantly alter the percent mineralization of the whole bone. Table III lists all the models that were developed with their associated modified variables.

Table III: Seven model variants were developed to analyze the impact that D-spacing has on bone mechanical properties. Two of the four variables in the staggered array model were constant and are not shown (collagen width and mineral plate width). Characters in bold have deviated from the control.

\begin{tabular}{|l|c|c|c|c|}
\hline \multicolumn{1}{|c|}{ Model Name } & $\begin{array}{c}\text { Periodic Unit } \\
\text { Length, } \mathrm{P} \\
(\mathrm{nm})\end{array}$ & $\begin{array}{c}\text { Mineral Volume } \\
\text { Fraction, } \\
\text { (unitless) }\end{array}$ & $\begin{array}{c}\text { Collagen } \\
\text { Length, } \mathrm{a} \\
(\mathrm{nm})\end{array}$ & $\begin{array}{c}\text { Mineral Length, } \\
\text { L } \\
(\mathrm{nm})\end{array}$ \\
\hline Normal D-spacing: Control & 67 & 0.3 & 21.44 & 112.56 \\
\hline $\begin{array}{l}\text { High D-spacing: Constant } \\
\text { Mineral Volume Fraction }\end{array}$ & $\mathbf{7 3}$ & 0.3 & $\mathbf{2 3 . 3 6}$ & $\mathbf{1 2 2 . 6 4}$ \\
\hline $\begin{array}{l}\text { Low D-spacing: Constant } \\
\text { Mineral Volume Fraction }\end{array}$ & $\mathbf{6 1}$ & 0.3 & $\mathbf{1 9 . 5 2}$ & $\mathbf{1 0 2 . 4 8}$ \\
\hline $\begin{array}{l}\text { High D-spacing: Constant } \\
\text { Mineral Dimensions }\end{array}$ & $\mathbf{7 3}$ & $\mathbf{0 . 2 7 5}$ & $\mathbf{3 3 . 4 4}$ & 112.56 \\
\hline $\begin{array}{l}\text { Low D-spacing: Constant } \\
\text { Mineral Dimensions }\end{array}$ & $\mathbf{6 1}$ & $\mathbf{0 . 3 3 0}$ & $\mathbf{9 . 4 4}$ & 112.56 \\
\hline $\begin{array}{l}\text { High D-spacing: Constant } \\
\text { Collagen Dimensions }\end{array}$ & $\mathbf{7 3}$ & $\mathbf{0 . 3 0 5}$ & 21.44 & $\mathbf{1 2 4 . 5 6}$ \\
\hline $\begin{array}{l}\text { Low D-spacing: Constant } \\
\text { Collagen Dimensions }\end{array}$ & $\mathbf{6 1}$ & $\mathbf{0 . 2 9 4}$ & 21.44 & $\mathbf{1 0 0 . 5 6}$ \\
\hline
\end{tabular}

The development of the composite bone model involved the creation of many model variants to explore the tools that Abaqus has to offer. This process required the ability to remove and correct warnings and errors associated with model standards. These cautions are important to understand and amend so that the finalized model can accurately describe the behaviors of the material. To view a detailed list of all the warnings and errors along with their solutions, refer to Appendix D.

\subsection{Custom Viscoelastic Material Definition}

As a material, bone can be viewed as a composite composed primarily of hydroxyapatite, collagen, and water. Hydroxyapatite on its own is virtually elastic; so the hydrated collagen in this model is treated as a viscoelastic material [44]. Rheological models provide a simple approach to model this viscoelasticity. The user subroutine in Abaqus, titled UMAT, provides a method to develop user defined material properties and allows for the implementation of rheological models into custom material 
property definitions [48]. The simplest rheological model that exhibits both stress relaxation and creep behaviors seen in viscoelastic materials was coded into the UMAT within Abaqus. This model is simply known as the standard linear solid and has two forms: the Maxwell body form and Kelvin-Voigt body form [3, 44] The Kelvin-Voigt body form of the standard linear solid was arbitrarily chosen to develop the viscoelastic material definition and is shown in Figure 18. There are no apparent advantages between the Kelvin-Voigt and Maxwell body forms as they both exhibit stress relaxation and creep.

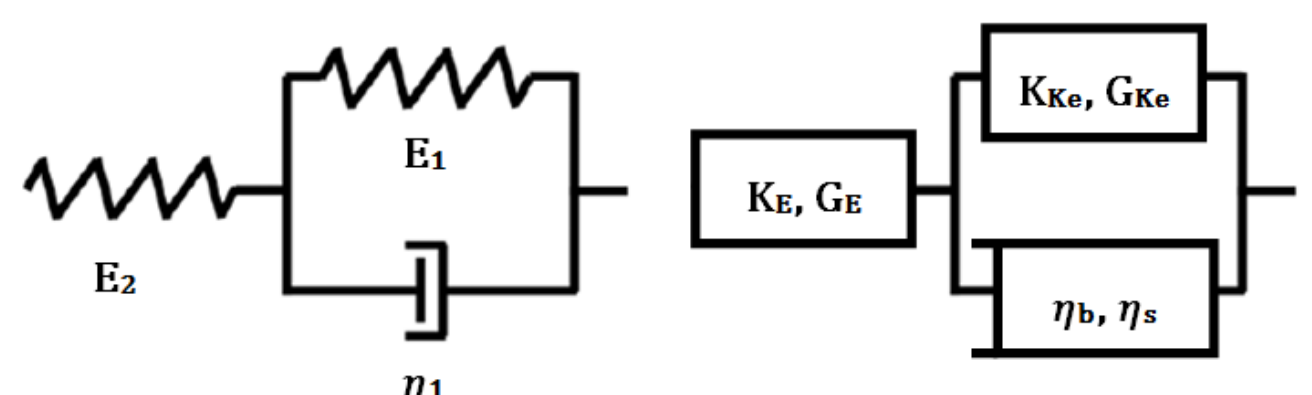

Standard Linear Solid

Figure 18: (Left) The Kelvin-Voigt body form of the standard linear solid model that is capable of exhibiting stress relaxation and creep viscoelastic behaviors [50]. (Right) The 3D version of the rheological model where the spring and dashpot elements are replaced by shear and bulk moduli.

Since rheological models are one dimensional formulations by definition, a custom form of the derivation was used. More specifically, the three dimensional form of the constitutive equation was used to develop the linear viscoelastic material definition [49]. The three dimensional form can be written as follows:

$$
\begin{aligned}
\left(1+\frac{G_{K e}}{G_{E}}\right) \sigma_{i j}+ & \left(\frac{K_{K e}}{K_{E}}+\frac{G_{K e}}{G_{E}}\right) \frac{\sigma_{k k}}{3} \delta_{i j}+\frac{\eta_{s}}{G_{E}} \dot{\sigma}_{i j}+\left(\frac{\eta_{b}}{K_{E}}+\frac{\eta_{s}}{G_{E}}\right) \frac{\dot{\sigma}_{k k}}{3} \delta_{i j} \\
& =2 G_{K e} \varepsilon_{i j}+\left(3 K_{K e}-2 G_{K e}\right) \frac{\varepsilon_{k k}}{3} \delta_{i j}+2 \eta_{s} \dot{\varepsilon}_{i j}+\left(3 \eta_{b}-2 \eta_{s}\right) \frac{\dot{\varepsilon}_{k k}}{3} \delta_{i j}
\end{aligned}
$$

The indices $i$ and $j$ represent the tensor component of the stress and strain tensors. The dot denotes a derivative with respect to time. The subscript $k k$ indicates the trace of the tensor (i.e. $\sigma_{\mathrm{kk}}=\sigma_{\mathrm{xx}}+\sigma_{\mathrm{yy}}+\sigma_{\mathrm{zz}}$ ) and $\delta_{i j}$ is the Kronecker delta. The spring and dashpot elements in the original standard linear solid model 
have been replaced by elastic and viscous coefficients in the 3D formulation, where the subscript ' $E$ ' indicates the lone spring element and 'Ke' stands for the spring element within the Kelvin-Voigt body. The elastic coefficients, $\mathrm{K}$ and $\mathrm{G}$, represent the bulk modulus and shear modulus respectively. The bulk and shear moduli are algebraic expressions composed of the spring elastic modulus (E) and Poisson's $\operatorname{ratio}(v)$.

$$
\begin{aligned}
& K=\frac{E}{3(1-3 v)} \\
& G=\frac{E}{2(1+v)}
\end{aligned}
$$

These relationships imply that there is an independent value for the elastic modulus and Poisson's ratio for each spring and thus independent bulk and shear moduli as well. Similarly, the viscous coefficients, $\eta_{\mathrm{b}}$ and $\eta_{\mathrm{s}}$, represent the bulk viscosity and shear viscosity. The expressions are nearly identical between the elastic and viscous coefficients with the exception of the viscosity, $\eta_{1}$, replacing the elastic modulus.

$$
\begin{aligned}
& \eta_{b}=\frac{\eta_{1}}{3\left(1-3 v_{\eta_{1}}\right)} \\
& \eta_{s}=\frac{\eta_{1}}{2\left(1+v_{\eta_{1}}\right)}
\end{aligned}
$$

If Poisson's ratio for each of the elements and the perpendicular components of the stresses and strains are set to zero, the three dimensional formulation simplifies down to the original one dimensional behavior for the standard linear solid.

$$
\sigma+\frac{\eta_{1}}{E_{1}+E_{2}} \dot{\sigma}=\frac{\eta_{1}}{1+\frac{E_{1}}{E_{2}}} \dot{\varepsilon}+\frac{1}{\frac{1}{E_{1}}+\frac{1}{E_{2}}} \varepsilon
$$

In order to create a user defined material in the UMAT from the governing equation, two major steps must be completed: the stresses and/or solution-dependent state variables must be updated at the end of the increment, and the material Jacobian matrix, $\partial \Delta \sigma / \partial \Delta \varepsilon$, must be defined [48]. The use of the 
following simple integration operator known as the central difference operator provides the avenue to accomplish these steps.

$$
\begin{gathered}
\dot{f}_{t+\frac{1}{2} \Delta t}=\frac{\Delta f}{\Delta \mathrm{t}} \\
f_{t+\frac{1}{2} \Delta t}=f_{t}+\frac{\Delta \mathrm{f}}{2}
\end{gathered}
$$

The variable, $f$, is an arbitrary function. $\Delta f$ represents the change of the function over the time increment, $\Delta t$, and $f_{t}$ is the value of the function at the beginning of the increment. Applying the central difference operator to the governing equation yields the following stress increments:

$$
\begin{gathered}
\Delta \sigma_{x x}=\mathrm{A} *\left[\left(G_{K e}+\frac{2 \mu_{K v}}{\Delta t}\right) \Delta \varepsilon_{x x}+\left(\frac{1}{6}\left(3 K_{K e}-2 G_{K e}\right)+\frac{1}{3 \Delta t}\left(3 \mathrm{~K}_{K v}-2 \mu_{K v}\right)\right) \Delta \varepsilon_{V}+2 \mathrm{G}_{K e} \varepsilon_{x x}\right. \\
+\frac{1}{3}\left(3 K_{K e}-2 G_{K e}\right) \varepsilon_{V}-\left(1+\frac{G_{K e}}{G_{E}}\right) \sigma_{x x}-\left(\frac{K_{K e}}{K_{E}}-\frac{G_{K e}}{G_{E}}\right) \frac{\sigma_{V}}{3}-\left(\frac{1}{6}\left(\frac{K_{K e}}{K_{E}}-\frac{G_{K e}}{G_{E}}\right)\right. \\
\left.\left.+\frac{1}{3 \Delta t}\left(\frac{K_{K v}}{K_{E}}-\frac{\mu_{K v}}{G_{E}}\right)\right)\left(\Delta \sigma_{y y}+\Delta \sigma_{z z}\right)\right] \\
\Delta \tau_{x y}=\mathrm{B} *\left[\left(\frac{G_{K e}}{2}+\frac{\mu_{K v}}{\Delta t}\right) \Delta \gamma_{x y}+G_{K e} \gamma_{x y}-\left(1+\frac{G_{K e}}{G_{E}}\right) \tau_{x y}\right]
\end{gathered}
$$

where the variables, A and B, represent constants composed of the viscous and elastic coefficients.

$$
\begin{gathered}
\mathrm{A}=\frac{6 \Delta t K_{E} G_{E}}{3 \Delta \mathrm{t} K_{E} G_{E}+2 \Delta t K_{E} G_{K e}+4 K_{E} \mu_{K v}+\Delta t G_{E} K_{K e}+2 G_{E} K_{K v}} \\
\mathrm{~B}=\frac{2 \Delta \mathrm{t} G_{E}}{\Delta \mathrm{t} G_{E}+\Delta t G_{K e}+2 \mu_{K v}}
\end{gathered}
$$

Finally, the Jacobian matrix was constructed from the following terms.

$$
\begin{gathered}
\frac{\partial \Delta \sigma_{x x}}{\partial \Delta \varepsilon_{x x}}=\mathrm{A} *\left[G_{K e}+\frac{2 \mu_{K v}}{\Delta t}+\frac{K_{K e}}{2}-\frac{G_{K e}}{3}+\frac{K_{K v}}{\Delta t}-\frac{2 \mu_{K v}}{3 \Delta t}\right] \\
\frac{\partial \Delta \sigma_{x x}}{\partial \Delta \varepsilon_{y y}}=\mathrm{A} *\left[\frac{K_{K e}}{2}-\frac{G_{K e}}{3}+\frac{K_{K v}}{\Delta t}-\frac{2 \mu_{K v}}{3 \Delta t}\right] \\
\frac{\partial \Delta \tau_{x y}}{\partial \Delta \gamma_{x y}}=B *\left[\frac{G_{K e}}{2}+\frac{\mu_{K v}}{\Delta t}\right]
\end{gathered}
$$


This linear viscoelastic material definition for the hydrated collagen in the composite model was coded into the UMAT user subroutine. The final version of the code can be viewed in Appendix E. Since the material definition is isotropic, the entire Jacobian matrix can be described by only three expressions.

\subsection{Parameter Identification}

The user defined material properties for collagen in the composite model are based on six different parameters. The first three coincide with the two springs and a single dashpot found in the standard linear solid. The next three variables are the three different Poisson's ratios related to each spring and dashpot. As stated before, if each of the Poisson's ratios are set equal to zero, the material definition simplifies down to a three dimensional version of the original standard linear solid. The obstacle that must be overcome involves establishing the value of all six parameters in order to more closely simulate the inherent mechanical properties of native collagen in compact bone [30]. The fact that springs and dashpots cannot be identified with physical features in a material adds to the difficulty [44]

The method used to determine the value for the variables involve the native mechanical properties of pure collagen tissue from literature $[1,2,4]$ and the measured tangent delta values from the ovine bending tests $[42,43]$. Since the Poisson's ratio of collagen tissue is approximately 0.2 , the three Poisson's ratios in the user defined material were also set to 0.2 . The collagen modulus of elasticity (2 GPa) was used to determine the values for both springs. The standard linear solid contains an equilibrium modulus of elasticity for both stress relaxation and creep. Because both viscoelastic behaviors are exponential functions, this equilibrium modulus occurs when the loading conditions are applied to the model for a sufficiently long time $(\mathrm{t} \approx \infty)$. In both cases, the equilibrium modulus of elasticity is a combination of both spring moduli: $E_{e q}=E_{1} E_{2} /\left(E_{1}+E_{2}\right)$. With an equilibrium modulus of $2 \mathrm{GPa}$, the values for the springs can be established $\left(E_{1}=3 G P a ; E_{2}=6 G P a\right)$.

Finally, a graphical approach was used to ascertain the magnitude of the dashpot. By running multiple simulations with varying dashpot values, the final amount was established through tangent delta 
comparisons. When the tangent delta of the model matched that of the control ovine data, the final value for the dashpot was determined. The mean tangent delta for the control animals was 0.018764 and a 1.25 GPa-s dashpot closely matched this number. Figure 19 reveals how the tangent delta was affected by a changing dashpot. The magnitude of the viscosity coincides with the magnitude used for the dimensions of the model $\left(0.001 \mathrm{~N}-\mathrm{s} / \mu \mathrm{m}^{2}=1 \mathrm{GPa}-\mathrm{s}\right)$.

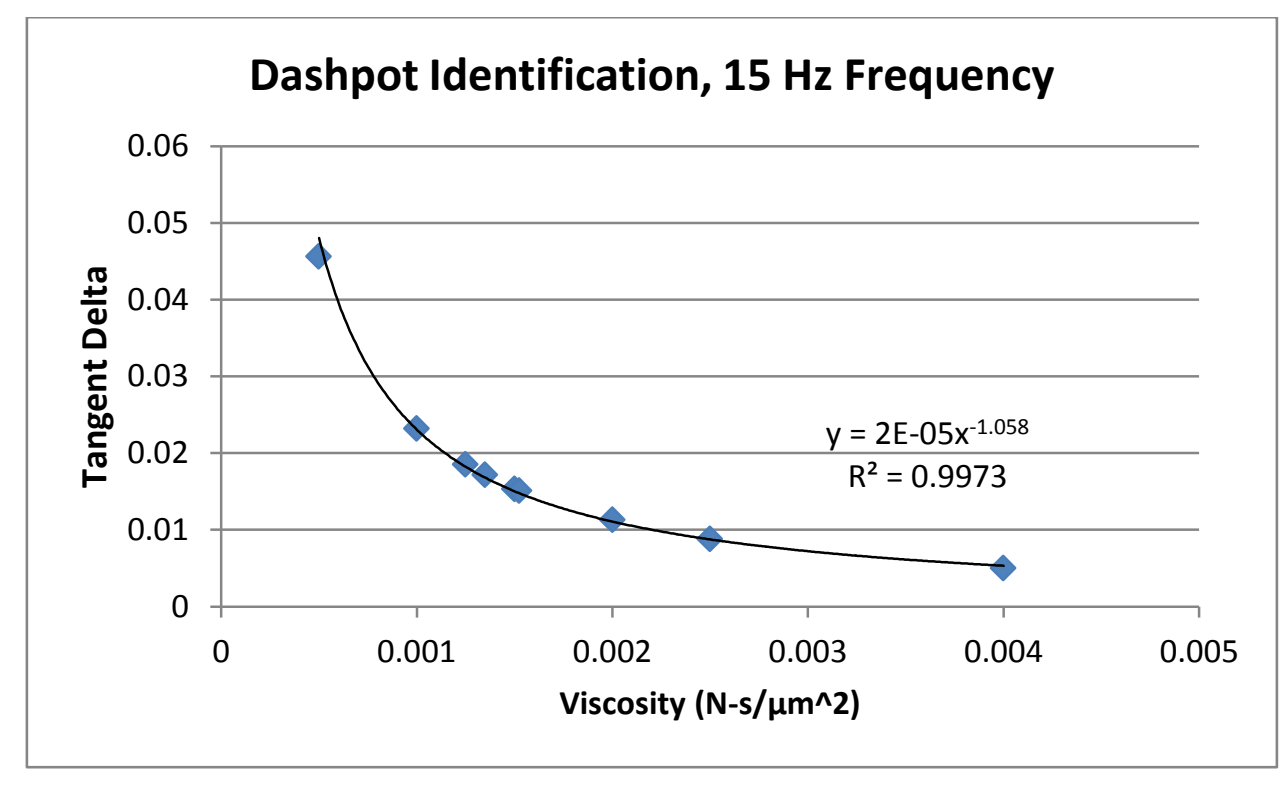

Figure 19: The behavior of the tangent delta with respect to the viscosity is described by the best fit equation on the plot. With this equation, the value for the dashpot was determined by solving for the tangent delta found in the control ovine animals.

\subsection{Mesh Development}

Abaqus contains a vast amount of element types that are used for specific situations. The plane strain conditions of this model narrowed down the number of elements to only a handful of choices. The axial loading conditions and simplified geometry of the model warrants the use of quadrilateral elements. These simple elements provide the accuracy and computational efficiency required by the analysis.

Additionally, quadratic quadrilateral elements were chosen over their linear analogue because the job run time was not significantly affected. Following the element type determination, the number of elements in the model was established with a test for convergence. 


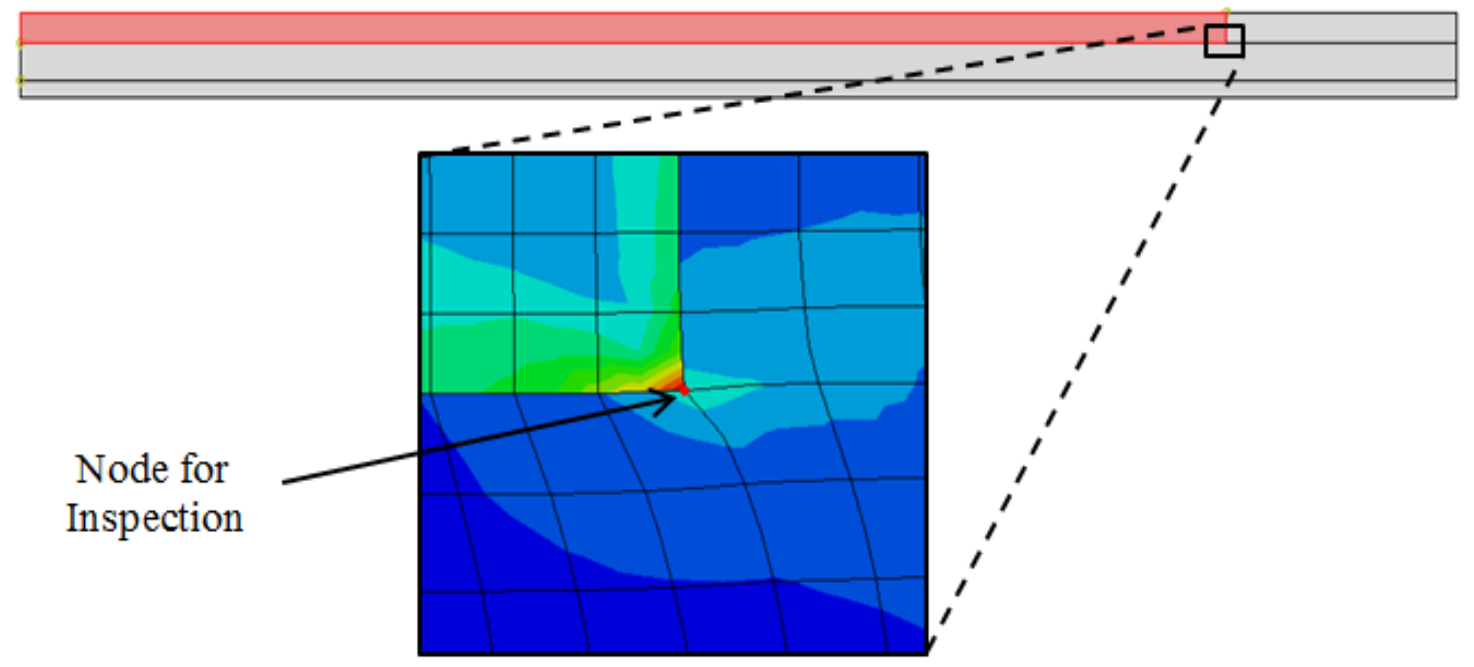

Figure 20: The test for convergence requires the inspection of a single node. The arrow points to the chosen node on the composite model. The zoomed in section reveals the stress distribution via the contour plotting tool in Abaqus.

The test for convergence spanned many iterations with alterations to the global seed size. This process allowed the model to be accurate without exhausting too much computing power. At the same time, the job run time was reduced to a manageable level. The convergence test involves the inspection of a specific element or node on the model to view the behavior of its associated stresses or strains. One particular node located between the HA material and collagen always had comparatively high levels of stress for each analysis, so this node was deemed the most important to determine an appropraite mesh seed size for the model. Figure 20 shows the significant node that was checked for convergence. The model was meshed with global seed sizes ranging from $5 \mathrm{~nm}$ to $0.25 \mathrm{~nm}$ and the resulting mesh convergence graph can be seen on Figure 21. Once the model reached the $0.75 \mathrm{~nm}$ seed size, the displacements began to oscillate at approximately 3.25e-6 microns indicating a termination of increased accuracy. From this analysis, an optimal mesh seed size of $0.45 \mathrm{~nm}$ resulted in approximately 10,000 degrees of freedom for the model. 


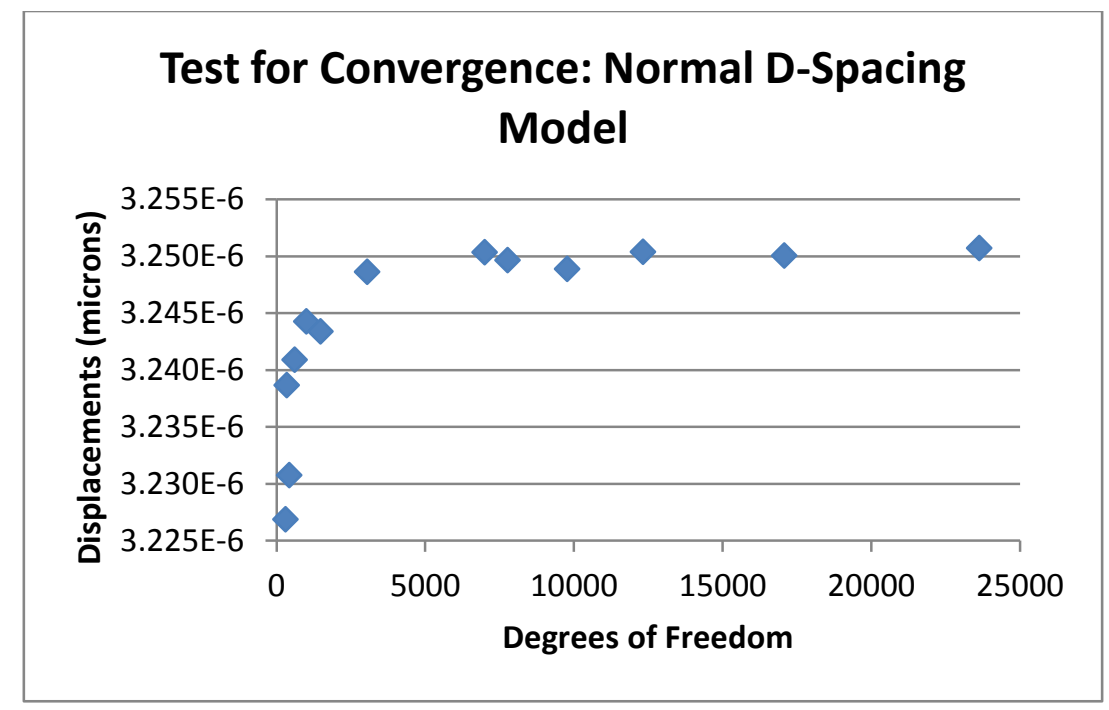

Figure 21: The finer the mesh becomes, the more precise the analysis becomes. The data points appear to plateau at around the 3.25e-6 micron value which indicates that smaller mesh seed sizes are unnecessary as accuracy would not be increased significantly.

Due to the simple geometry of the model, the quality of elements was very high. No errors or warnings occurred that pertained to the $\min / \max$ angle and aspect ratio criteria that Abaqus requires. Similar analyses for each of the composite model variants were executed yielding the same optimum mesh seed size of $0.45 \mathrm{~nm}$.

\subsection{Model Validation}

Before running the models to analyze the results, the user defined material was validated against standard linear solid calculations. Since the rheological model exhibits stress relaxation and creep, it is of interest to see how the user defined material compares to these viscoelastic behaviors. The UMAT code was applied to a one dimensional truss element. All the Poisson's ratios were set equal to zero effectively simplifying the material definition down to the original spring and dashpot network. The springs and dashpot were then set equal to arbitrary values (100 Pa, $100 \mathrm{~Pa}, 100 \mathrm{~Pa}-\mathrm{s})$. The stress relaxation and creep tests were then applied to the model separately and the resulting stresses and strains were recorded.

The governing equation for the Kelvin-Voigt body form of the standard linear solid was then manipulated to solve for either stress relaxation or creep. Both viscoelastic behaviors yield an exponential 
equation with the same equilibrium modulus but differing time constants. The specifics of the calculations can be seen in Appendix F. Using arbitrary time points, the calculations were compared to the Abaqus simulation. The stresses for the stress relaxation test and strains for the creep test were nearly identical. This revealed that, at the very least, the custom viscoelastic material definition was validated against the one dimensional standard linear solid.

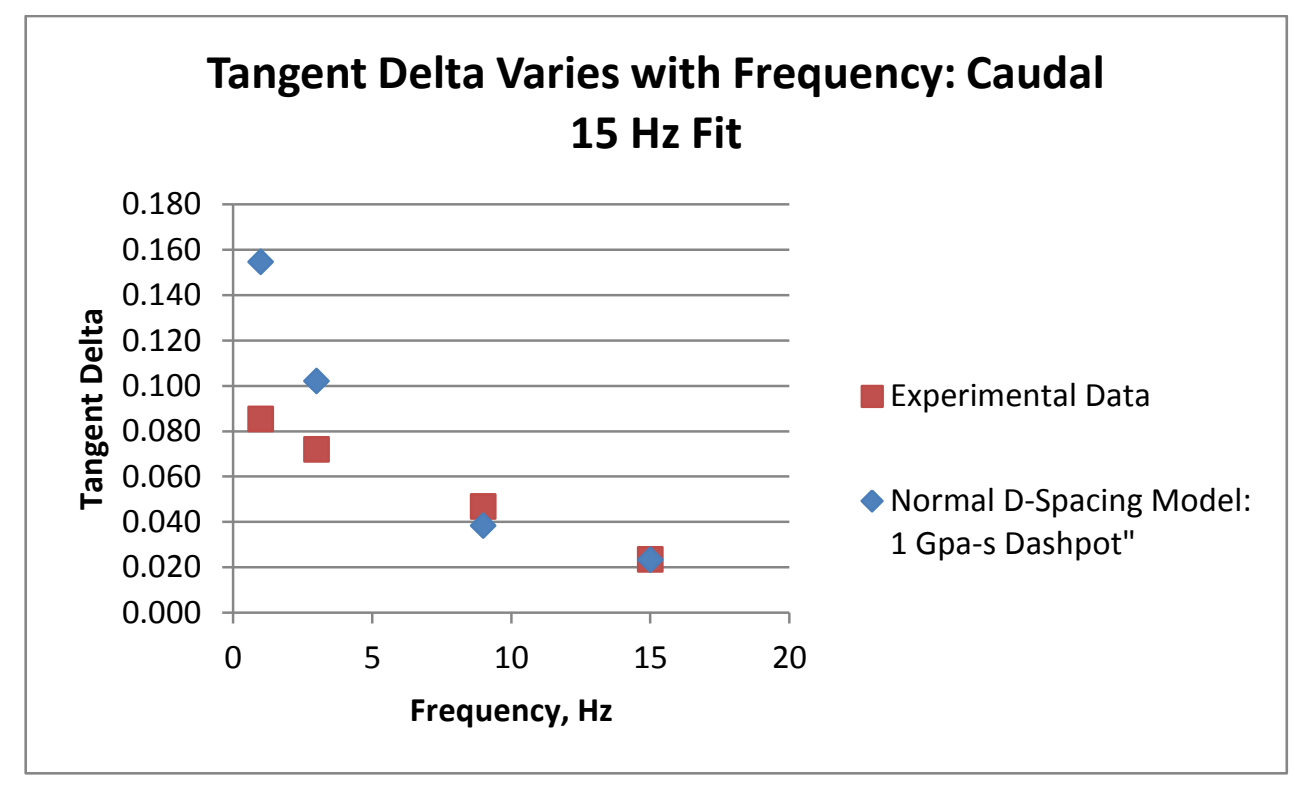

Figure 22: Graphical comparison between the experimental ovine data and the results from the Normal D-spacing variant of the composite model. The data has a coefficient of determination $\left(\mathrm{r}^{2}\right)$ of 0.918 .

The results of the composite model were then compared to the experimental viscoelastic data, namely the tangent delta. The values were specifically compared at the frequencies of interest: $1,3,9$, and $15 \mathrm{~Hz}$. Figure 22 reveals a reasonable fit at the higher frequencies of the composite model, but certainly deviates from the experimental data at the low frequencies. The possible causes for the resulting fit are described in detail in the discussion section.

\subsection{Post Processing}

The Abaqus simulations produce stresses, strains, and more at all the nodes and elements of the composite model. In order to evaluate the viscoelastic properties of the model, the deflections at specific nodes must be gathered from the output database or .odb file that is produced from running an Abaqus 
job. The only way to access information from the output database is through Abaqus CAE or Python. While CAE allows the user to view data from specific nodes and elements, only Python can facilitate the extraction of the data for further use outside of Abaqus. A python code was written to gather the deflections from the nodes at the loaded end of the composite model. The deflections at each of the nodes were automatically written to data files which could then be processed through MATLAB.

The MATLAB code was written to convert these nodal deflections into composite model strains employing the periodic unit length of the model. More specifically, the deflections of these nodes were averaged at each time point. The average deflection was then divided by the periodic unit length to solve for the axial strain of the model $\left(\varepsilon=\Delta L / L_{o}\right)$. The strain history was then plotted to determine the phase shift or tangent delta of the simulation. This was done by utilizing a best fit algorithm to fit a sinusoidal equation to the strain data. The python script and MATLAB code can be viewed in Appendices $\mathrm{G}$ and $\mathrm{H}$ respectively. 


\section{RESULTS}

The viscoelastic properties of the ovine experimental data were measured in the form of tangent delta, where tangent delta is essentially the tangent of the phase shift between the loading and deformation curves. The DMA tests were performed at frequencies ranging from 1 to $20 \mathrm{~Hz}$; however, only four different frequencies were compared against the FE model data $(1,3,9$, and $15 \mathrm{~Hz})$. The data is categorized based on treatment type, anatomical location, and frequency of the DMA tests. A table of the raw data is located in Appendix A. Table IV displays the mean tangent delta values from the experimental data. The data from tables V and VI are derived from the FE models.

Table IV: Experimental ovine DMA data at varying frequencies. The control animals underwent a sham surgery leaving the endocrine organs intact.

\begin{tabular}{|l|c|c|c|c|}
\cline { 2 - 5 } \multicolumn{1}{c|}{} & \multicolumn{3}{c|}{ Frequency (Hz) } \\
\cline { 2 - 5 } \multicolumn{1}{c|}{} & 1 & 3 & 9 & 15 \\
\hline Animal Model & $\begin{array}{c}\text { Mean Tangent } \\
\text { Delta }\end{array}$ & $\begin{array}{c}\text { Mean Tangent } \\
\text { Delta }\end{array}$ & $\begin{array}{c}\text { Mean Tangent } \\
\text { Delta }\end{array}$ & $\begin{array}{c}\text { Mean Tangent } \\
\text { Delta }\end{array}$ \\
\hline Cranial: Control & 0.076557 & 0.064371 & 0.039474 & 0.018764 \\
\hline Caudal: Control & 0.085354 & 0.071957 & 0.046737 & 0.023365 \\
\hline Mean Control & 0.080955 & 0.068164 & 0.043106 & 0.021064 \\
\hline Cranial: OVX & 0.07302 & 0.06217 & 0.03886 & 0.016626 \\
\hline Caudal: OVX & 0.079202 & 0.066225 & 0.040936 & 0.016758 \\
\hline Mean OVX & 0.076111 & 0.064197 & 0.039898 & 0.016692 \\
\hline
\end{tabular}

The initial analysis focused on the cranial side of the experimental ovine data. By realizing that the D-spacing distribution on this anatomical side showed an overall decrease in D-spacing length due to the surgically induced menopause (Figure 14), it was decided to simulate this decrease with the low Dspacing model variants. In order for the normal D-spacing model to yield a similar tangent delta value to the caudal control ovine data, the model would require a dashpot with $4 \mathrm{GPa}-\mathrm{s}$ viscosity at the $1 \mathrm{~Hz}$ frequency and a $1.25 \mathrm{GPa}$-s dashpot at the $15 \mathrm{~Hz}$ frequency. The model containing the $4 \mathrm{GPa}$-s dashpot had a relatively weak coefficient of determination $\left(r^{2}=0.746\right)$ to the experimental data; however, the 1.25 GPa-s dashpot resulted in a better fit to the experimental data $\left(\mathrm{r}^{2}=0.874\right)$. It is particularly noticeable in the higher frequencies (Figure 23). 


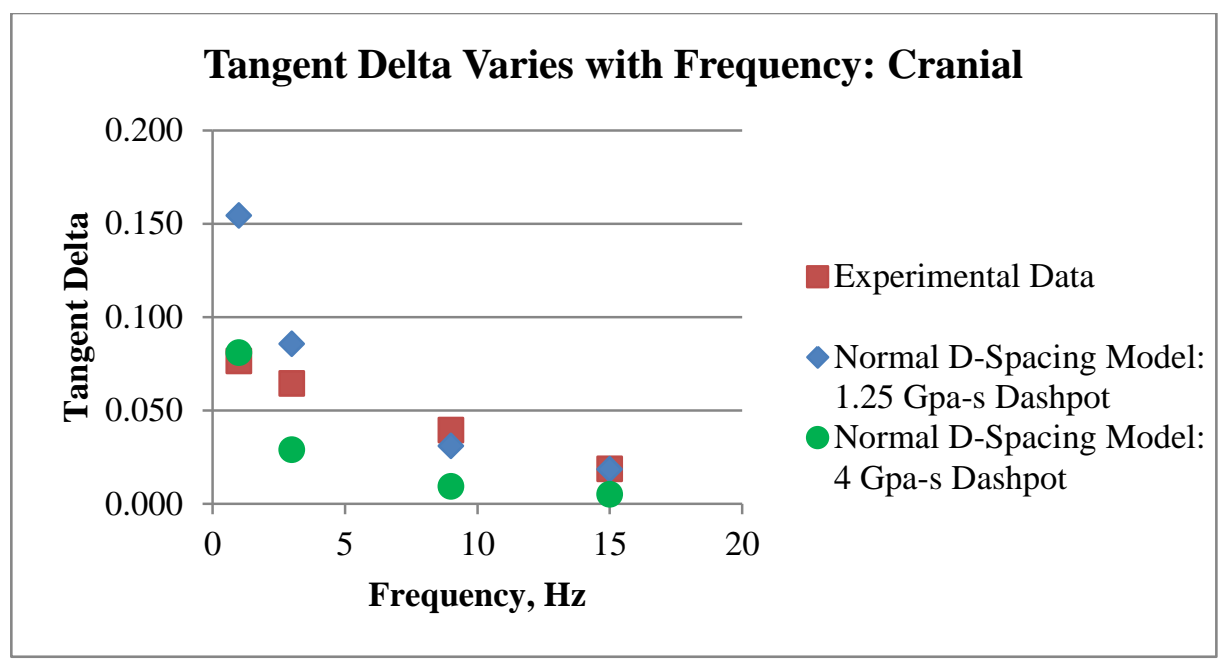

Figure 23: A plot of the viscoelastic properties for the DMA tests at varying frequencies. A dashpot value of $4 \mathrm{GPa}$-s for the FE model resulted in a less than desirable fit $\left(\mathrm{r}^{2}=0.746\right)$ to the experimental data. A dashpot value of $1.25 \mathrm{GPa}$-s for the model has a better fit $\left(\mathrm{r}^{2}=0.874\right)$ to the experimental data.

Table V displays the FE model data along with the target tangent delta from the experimental ovine data. The experimental data revealed that the tangent delta decreased following ovariectomy on the cranial side for the 1 and $15 \mathrm{~Hz}$ frequencies. For the $1.25 \mathrm{GPa}$-s dashpot data, only the constant mineral dimension model variant shows a significant decrease in tangent delta. It's worth noting that the model with a $4 \mathrm{GPa}$-s dashpot also had a significant decrease in the tangent delta for the constant mineral dimension variant to surpass the behavior displayed by the OVX data.

Table V: Composite bone FE model data for the cranial side of the ovine bone. The model for the $1 \mathrm{~Hz}$ test has a dashpot of $4 \mathrm{GPa}$-s and the model for the $15 \mathrm{~Hz}$ test has a dashpot of $1.25 \mathrm{GPa}$-s. The target tangent delta originates from the experimental data.

\begin{tabular}{|c|c|c|c|c|}
\hline & \multicolumn{2}{|c|}{$1 \mathrm{~Hz}$ Test } & \multicolumn{2}{|c|}{$15 \mathrm{~Hz}$ Test } \\
\hline Model & $\begin{array}{c}\text { Target Tan } \\
\text { Delta }\end{array}$ & Tangent Delta & Target Tan Delta & Tangent Delta \\
\hline Normal D-spacing & 0.076557 & 0.0808910 & 0.018764 & 0.0185500 \\
\hline $\begin{array}{l}\text { Low D-spacing: } \\
\text { Constant Mineral } \\
\text { Volume Fraction }\end{array}$ & \multirow{3}{*}{0.07302} & 0.0811030 & \multirow{3}{*}{0.016626} & 0.0186090 \\
\hline $\begin{array}{l}\text { Low D-spacing: } \\
\text { Constant Mineral } \\
\text { Dimensions }\end{array}$ & & 0.0640830 & & 0.0145910 \\
\hline $\begin{array}{l}\text { Low D-spacing: } \\
\text { Constant Collagen } \\
\text { Dimensions }\end{array}$ & & 0.0843100 & & 0.0190010 \\
\hline
\end{tabular}




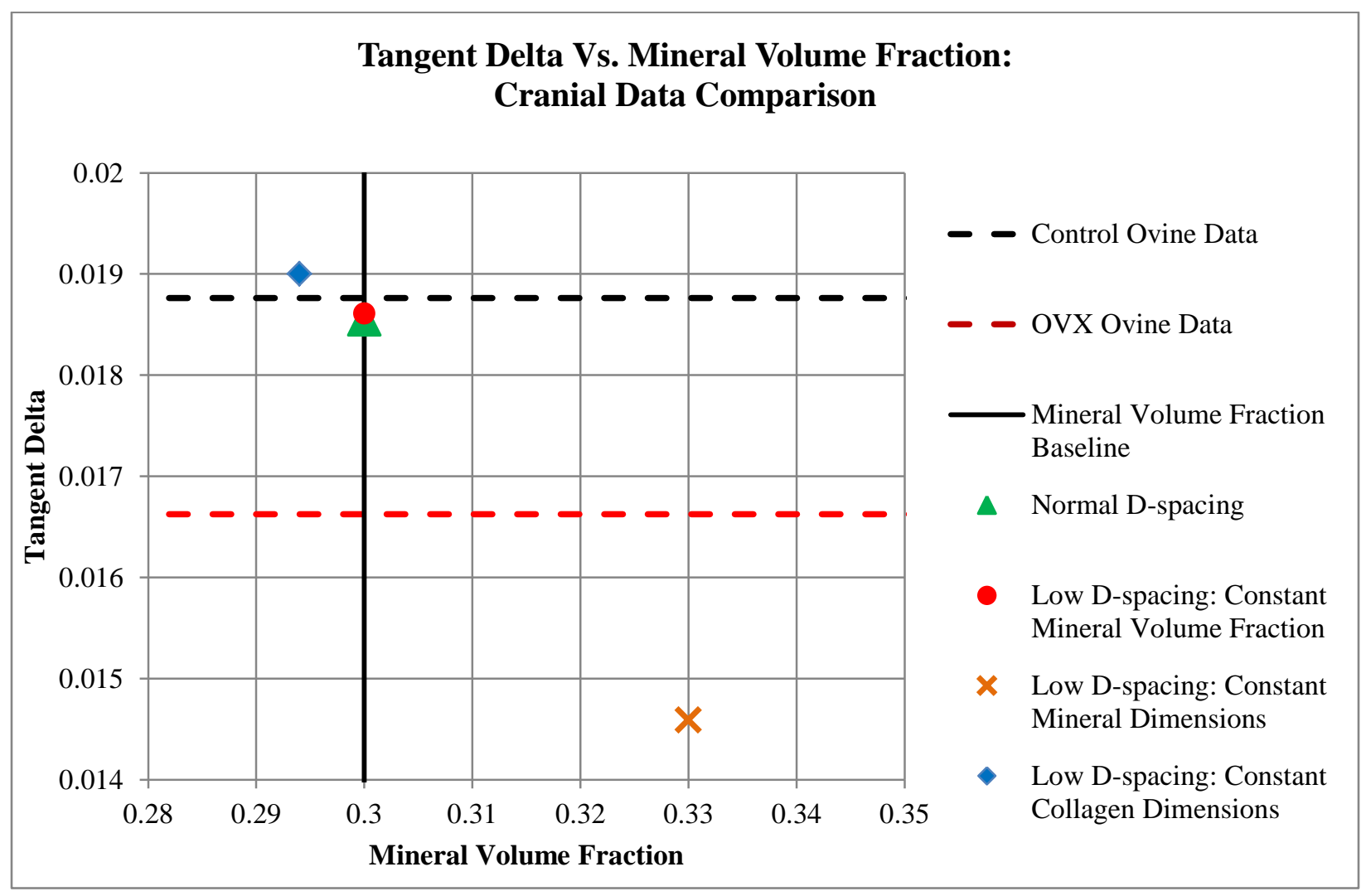

Figure 24: The viscoelastic data of the $15 \mathrm{~Hz}$ frequency tests are graphically represented here to reveal the relationship between tangent delta and mineral volume fraction.

When the viscoelastic data is graphically shown against the mineral volume fraction of the composite model (Figure 24), their relationship is more easily understood. A substantial increase in mineral volume fraction $(+10 \%)$ corresponds to a significant decrease in tangent delta $(-21.3 \%)$ for the constant mineral dimension model. The constant collagen dimension model contained a slight decrease in mineral volume fraction $(-2 \%)$ and small increase in tangent delta $(+2.4 \%)$. For clarification, the percentages are derived from a comparison to the normal D-spacing model. 


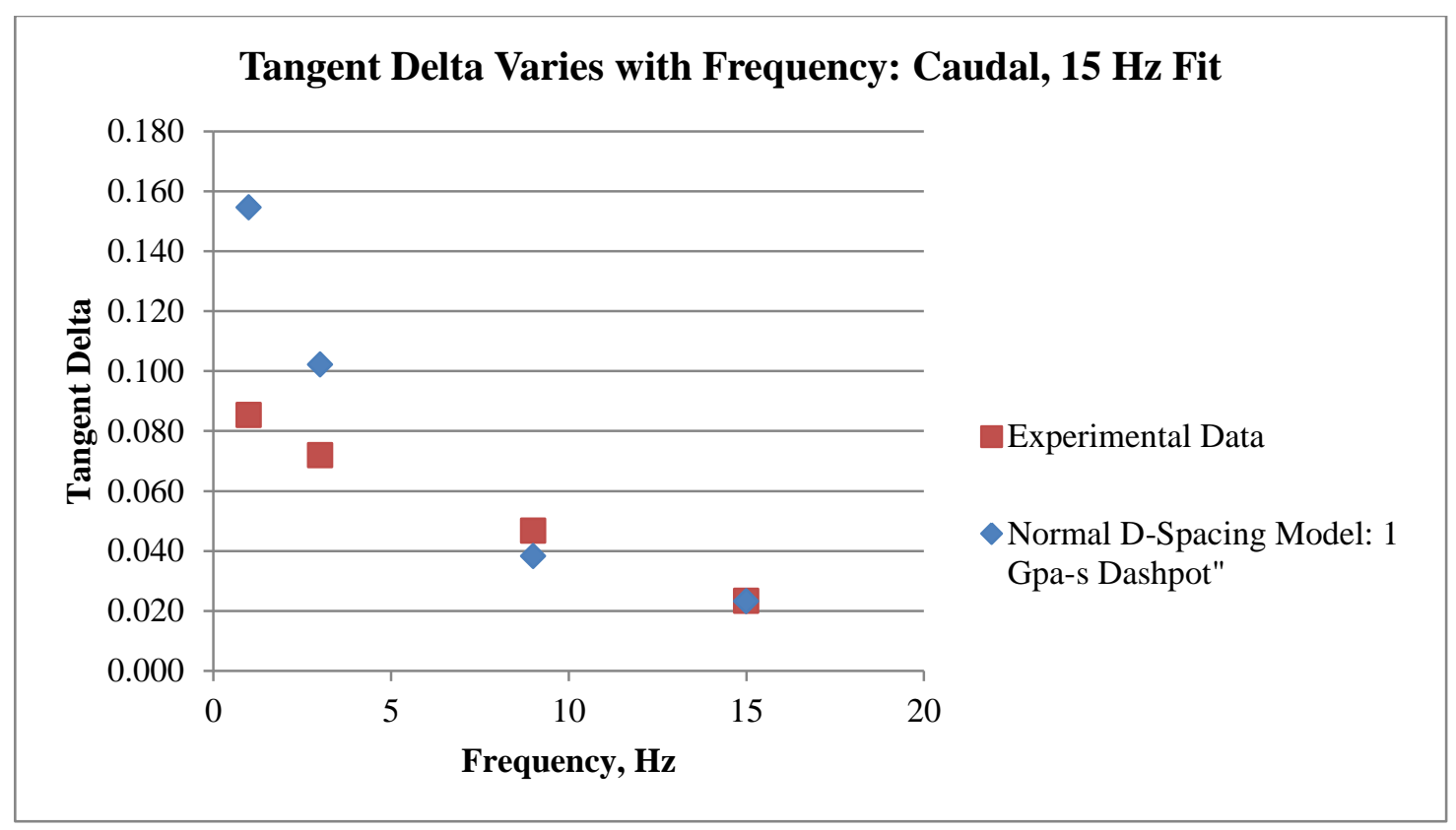

Figure 25: The viscoelastic properties of the FE model and experimental ovine data are displayed as a function of frequency. The $1.25 \mathrm{GPa}-\mathrm{s}$ dashpot yielded a $\mathrm{r}^{2}$ of 0.918 .

Table VI: Composite bone FE model data for the caudal side of the ovine bone using the $1 \mathrm{GPa}-\mathrm{s}$ dashpot. The target tangent delta originates from the experimental data.

\begin{tabular}{|l|r|r|}
\cline { 2 - 3 } \multicolumn{1}{c|}{} & \multicolumn{2}{c|}{$15 \mathrm{~Hz}$ Test } \\
\hline Normal D-spacing & Target Tan Delta & Tangent Delta \\
\hline $\begin{array}{l}\text { High D-spacing: Constant } \\
\text { Mineral Volume Fraction }\end{array}$ & 0.023365 & 0.0231760 \\
\hline $\begin{array}{l}\text { High D-spacing: Constant } \\
\text { Mineral Dimensions }\end{array}$ & & 0.0231060 \\
\hline $\begin{array}{l}\text { High D-spacing: Constant } \\
\text { Collagen Dimensions }\end{array}$ & & 0.0253970 \\
\cline { 1 - 1 } & & 0.0225170 \\
\hline
\end{tabular}

An additional analysis took place to utilize the caudal ovine data as well. The basic design of this analysis is essentially the same. The high D-spacing model variants were used in this case to explore the increasing D-spacing distribution seen on the caudal side from the previous experimentation (Figure 14). The viscosity of the dashpot required to yield similar viscoelastic properties to the control data was 1.0 GPa-s. The resulting FE model data had a strong relationship to the experimental data $\left(\mathrm{r}^{2}=0.918\right)$ as 
shown in Figure 25. The results differed in this analysis, the only model variant causing a decrease in the tangent delta was the constant collagen dimension one.

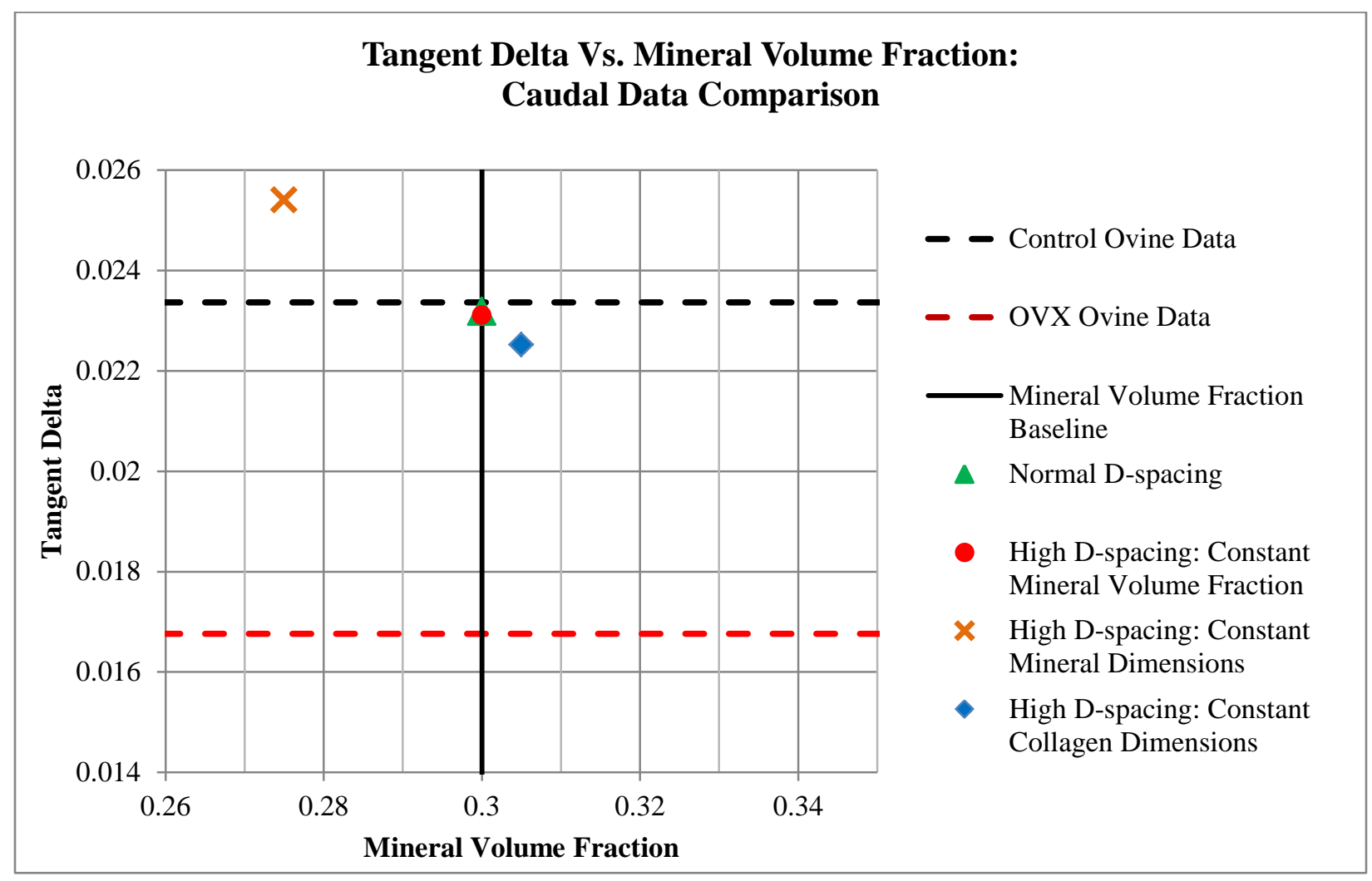

Figure 26: The viscoelastic data of the $15 \mathrm{~Hz}$ frequency tests are graphically represented here to reveal the relationship between tangent delta and mineral volume fraction.

The tangent delta and mineral volume fraction relationship seen in the cranial data is maintained in the caudal data (Figure 26). Only the tangent delta of the constant collagen dimension model decreased toward the ovine OVX experimental data $(-2.8 \%)$. The increase in mineral volume fraction was also minimal $(+1.7 \%)$. The constant mineral dimension model variant had a greater deviation in mineral volume fraction from the baseline measurement of the normal D-spacing model (-8.3\%). The tangent delta also underwent a more substantial change compared to the other model variants $(+9.6 \%)$. Once again, these percentages stemmed from a comparison to the Normal D-spacing model variant. 


\section{DISCUSSION}

Osteoporosis is a serious bone ailment that affects millions of people around the world. As the human population continues to live longer due to advancements in health care, osteoporosis becomes increasingly more common in the elderly population, and especially in women. This progressive loss of bone mass increases fracture risk and even minor traumatic events can lead to bone fractures in severe osteoporosis cases. These osteoporosis related fractures can have lasting detrimental effects on the quality of life. Even more worrisome, is the tendency for osteoporosis diagnoses to occur subsequent to osteoporotic fractures $[5,52]$. While the current osteoporosis screening technique to measure BMD with dual-energy X-ray absorptiometry (DXA) is effective at determining fracture risk based on bone density, additional biological aspects contribute to fracture risk that aren't measured. In fact, most fractures occur in individuals who have higher BMD values than the osteoporosis threshold [77].

To strengthen the fracture risk assessment that is imparted by BMD measurements, additional risk factors associated with osteoporosis such as prior bone fractures, age, sex, body mass index, smoking and alcohol intake, and many others were incorporated into predictive algorithms. There are many of these tools and countries all over the world contain their own standards and regulations that create increased variability in how each algorithm determines fracture risk. One such system, called the fracture risk assessment tool (FRAX), has at least 35 different models [77]. With so many developing predictive tools, it's clear that much work is still required to manage-treat individuals with osteoporosis and ultimately reduce the economic impact of the disease.

In addition to these previously described risk factors, micro-damage, cortical and trabecular architecture, mineralization, and changes to the biochemistry of collagen all affect the strength and toughness of the whole bone. This is where the use of animal and computational models is extremely beneficial. The effects that OVX, or other osteoporosis/osteopenia inducing method, has on bone tissue and its mechanical properties, remodeling characteristics, structural architecture, biochemistry, and more can be measured directly with animal models or indirectly with computational ones. Obviously with 
animal models, the exact human biology cannot be mimicked and careful analysis of the characteristics of each species must be taken into account prior to the actual experimentation.

The ovine species definitely has its strengths and weaknesses in osteoporosis related research just as every other animal model. The ewe in particular has become a widely used large animal model to study the effects that estrogen depletion has on bone metabolism, mechanical integrity, and structure. By surgically removing the ovaries, osteopenia can be induced in these animals due to the increased remodeling activity that is also seen in postmenopausal women. The similar estrogen profile between ewes and women advocates their use; however, ewes are predominantly polyestrous breeders leading to seasonal variations that cannot be ignored. Additionally, differences in the ewe's digestive system complicate the investigation of some osteoporosis treatments. Despite these distinctions: these animals are economical and easy to manage; their large skeletal size encourages mechanical testing, medical device implantation, and fracture healing studies; their extensive Haversian remodeling provides a means to research bone turnover and its relationship to osteoporosis [71].

Of course, experimentation utilizing animal models is not only limited by the biological differences between the species. Previous postmenopausal osteoporosis research on ewes has shown significant alterations to the D-spacing distributions and viscoelastic properties of the whole bone $[7,8$, $42,57]$; however, the ability to physically measure the mechanical implications of D-spacing on the whole bone structure is currently not possible. The scale of such architectural behaviors is simply too small to mechanically test with modern equipment. Thus, the use of finite element analysis and other computational means provide an alternative approach to examine such queries. In this case, a simplified plane strain analysis utilizing the staggered array model of Jager and Fratzl incorporated D-spacing as an adjustable variable in the form of the periodic unit length of the model [12]. Multiple model variants were required to maintain the mathematical relationship between the periodic unit length and the rest of the variables. In this way, the possible mechanisms causing changes to the D-spacing distributions of the osteopenic ewes could be explored. 
The preliminary results from the analysis seem to indicate that D-spacing on its own does not significantly alter the viscoelastic properties of the material as seen by the constant mineral volume fraction model variant. This model variant essentially maintains the mineral volume fraction by either increasing or decreasing the lengths of the mineral and collagen domains depending on how D-spacing changes: if the D-spacing length increases, both the collagen and mineral lengths increase and vice versa. The tangent delta is virtually unchanged compared to the normal D-spacing model in all three cases (Tables $\mathrm{V}$ and a). The results from the other model variants describe a different situation. In both cases, the mineral volume fraction is free to deviate from the initial value and either the collagen or mineral length is held constant. The tangent delta is significantly altered only when the mineral volume fraction changes. This behavior is in line with the previous observation that changes in bone mineral content affects the viscoelastic properties of the structure. The finding was attributed to bone remodeling and how partially mineralized tissue is introduced to the bone structure [78]. It also makes sense from the FE analysis standpoint: the HA crystals are modeled as perfectly elastic and the organic domain, collagen and water, is viscoelastic. So, changes to the mineral volume fraction should affect the viscoelastic properties of the composite material.

The anatomical variability in the ewe data encouraged the notion to treat the cranial and caudal sides of the bone as two separate materials. The D-spacing distributions either increased or decreased to result in a material that appeared to become more homogeneous due to estrogen depletion [7]. The FE caudal data revealed that the increase in D-spacing of the constant collagen model variant resulted in the decrease of tangent delta that was seen in the OVX experimental data. This may suggest that changes to D-spacing distribution in bone tissue are caused by a mechanism that alters the length of the mineral crystals. The biggest issue with this result is that the changes to the FE data were not as significant as the OVX data, which could be an indication that $\mathrm{D}$-spacing is only part of the reason that viscoelastic properties change with OVX. 
In the other anatomical sector, the D-spacing distribution decreased following ovariectomy. The low D-spacing model variants for the cranial data had quite different results than the caudal data. The constant mineral dimension model in this case produced lower tangent delta values coinciding with the OVX experimental data. In fact, the tangent delta affects were more significant and even surpassed the ovine data. In essence, the results of the cranial data suggest quite the opposite of the caudal data. Dspacing is altered from changes in collagen, not mineral, lengths. These contradictory results could imply that there are two very different mechanisms causing the D-spacing changes on the anatomical sides, or it could be a product of a very different mechanism occurring in the bone tissue. Upon further inspection, the model variants contained interesting deviations in mineral volume fractions. Wherever there was a marked alteration to the mineral volume fraction, significant changes to the tangent delta also transpired. A 10 percent increase in mineral volume fraction coincided with a 21.3 percent decrease in tangent delta in the constant mineral model of the cranial data; whereas, a 1.7 percent increase in mineral volume fraction yielded a 2.8 percent decrease in tangent delta in the constant collagen model of the caudal data. Once again, these are strong indications that the mineral content in bone results in the greatest changes to the viscoelastic properties of the structure.

So, the major findings of these results are that D-spacing might indeed play a significant role in the changing viscoelastic properties of the whole bone; however, these changes must also be accompanied by alterations in the mineral volume fraction. Taking the scale of the model into account, the composite arrangement of the HA mineral crystals and collagen can only appropriately be compared to that of a single mineralized collagen fibril. Obviously, the viscoelastic properties of this model were compared to machined beams of compact bone that are much larger in scale than a single collagen fibril. Thus, many structural complexities including varying orientations of collagen fibrils, Haversian systems, pores, mineral, and lamellae were overlooked for the sake of analyzing the D-spacing behavior of type I collagen. The extrafibrillar mineral is a noteworthy aspect in bone that was not accounted for in the 
staggered array model as well. Because of this, important conjectures on the previous conclusions could be made.

It was previously determined that alterations to the viscoelastic properties of the whole bone do not require significant changes to the mineral content [42]. The results of this model suggest that mineral volume fraction does indeed affect the tangent delta of the collagen fibril; however, this can only realistically be attributed to the intrafibrillar mineral. Another study revealed that the amount of extrafibrillar mineral is much greater than intrafibrillar [13]. Therefore, it is possible that the intrafibrillar mineral content could change enough to significantly impact the viscoelastic characteristics of the whole bone, without significantly altering the overall mineral content. This notion cannot be validated with this particular computational model, which warrants the continued investigation of D-spacing as well as intrafibrillar versus extrafibrillar mineral and their impact on the mechanical behavior of bone tissue.

One of the limitations of the computational model that was previously discussed involves the simplification of the structural arrangement of compact bone tissue. To build on this concept, the FE model was also loaded along the long axis of the mineralized collagen fibril. With changing collagen fibril orientations occurring in the lamellar structures of the tissue, it's obvious that the computational loading conditions are idealized for the sake of simplicity. A more realistic approach would be to ascertain the collagen fibril orientations of each bone specimen and apply loading conditions to a 3 dimensional model with matching orientations of staggered array models within. This is no easy task and an even more simple preliminary approach could involve loading the staggered array model off axis to investigate the possible implications. The type of loading condition, however, is more justifiable, since in vivo strain gage measurements of the ovine radius and ulna bone suggested a bending moment as the primary load during normal activity [75]. At the nano-scale of the compact bone tissue, this loading type would appear to be tensile or compressive axial forces.

Another limitation of this analysis has to do with the mechanical properties of collagen. In this case, the elastic properties of tendon were used to determine the properties of the hydrated collagen in the 
composite model. This method is typically used because tendon is primarily composed of type I collagen. This doesn't mean the method is necessarily accurate, however. The properties of tendon could be vastly different from the native mechanical properties of collagen [1]. With current technology, it's impossible to measure the mechanical properties of individual collagen fibrils, so the use of such an approximation is often warranted. In addition, the use of the standard linear solid to model the time dependent behavior of collagen is a simple initial approach that limits the flexibility and accuracy of the FE model. The complexity of the spring and dashpot model can be increased with the inclusion of additional elements. Or a prony series expansion of the standard linear solid could be used to more accurately model the experimental data. The mechanical properties of the collagen in this analysis are also assumed to be constant between the control and altered D-spacing models. It's entirely possible that estrogen depletion causes the intrinsic mechanical properties of collagen to change just as ageing does through alterations of the collagen biochemistry [28, 29].

As far as the experimental ovine data is concerned, the sample size is relatively small. While there were a total of 24 bone specimens that had undergone the DMA testing, the samples were harvested from only 6 different sheep. As with any analysis, the greater the number of samples, the greater statistical significance the data will have. The findings of this study can be strengthened with greater sample sizes in the experimental data and future studies should also incorporate D-spacing distributions of collagenous tissues. With so many findings revealing the D-spacing distribution behavior [8, 57, 58], it would be beneficial to model these distributions for more accurate representations of biological materials. More specifically, the modeling of homogeneous versus heterogeneous D-spacing distributions of compact bone tissue may provide insight into the changes seen in previous ovine experimentation [7]. 


\section{CONCLUSION}

Ewes are an economical and versatile large animal model for postmenopausal osteoporosis. Their polyestrous breeding behavior, unique gastrointestinal system, and comparatively high BMC and BMD values are differences that cannot be ignored; however, the ability to ovariectomize and induce osteopenia on these large docile animals encourages their use in histomorphometry, device implantation, fracture healing studies, and mechanical testing. With significant alterations occurring to the D-spacing distributions following ovariectomy of ewes, it is desirable to understand the mechanism or mechanisms that cause this microscopic structural change and the possible mechanical implications on the whole bone.

The results of this research seem to indicate that changes to D-spacing lengths are capable of altering the viscoelastic characteristics of bone tissue. The models for the cranial and caudal analyses caused significant deviations to the tangent delta only when the bone mineral volume fraction was significantly altered. If the intrafibrillar mineral content must deviate in order to accommodate the Dspacing shift, then the viscoelastic properties of the individual collagen fibrils located throughout the compact bone tissue could be affected.

Because of this preliminary approach utilizing the 2 dimensional staggered array model for the composite arrangement of a mineralized collagen fibril and basic standard linear solid model to represent collagen viscoelasticity, it is imperative to strengthen the validity and accuracy in future studies by increasing the complexity of the computational method. Implementation of a 3 dimensional model, the inclusion of structural complexities like alternating collagen fibril orientations and D-spacing distributions, and the employment of a more robust viscoelastic material definition for collagen are all important aspects that analyses of this nature can benefit from looking forward. 


\section{REFERENCES}

1. Martin, R. Bruce, David B. Burr, and Neil A. Sharkey. Skeletal Tissue Mechanics. New York: Springer, 1998.

2. Currey, John D. Bones: Structure and Mechanics. Princeton, NJ: Princeton UP, 2002.

3. Ethier, Christopher Ross, and Craig A. Simmons. Introductory Biomechanics: From Cells to Organisms. Cambridge: Cambridge UP, 2007.

4. Bartel, Donald L., Dwight T. Davy, and Tony M. Keaveny. Orthopaedic Biomechanics: Mechanics and Design in Musculoskeletal Systems. Upper Saddle River, NJ: Pearson/Prentice Hall, 2006.

5. "What Is Osteoporosis?" Nof.org. National Osteoporosis Foundation, n.d. Web. 11 Feb. 2013.

6. Michels, E. M., C. L. Pechey, J. M. MacLeay, M. J. Mager, A. S. Turner, and C. M. Les. "The Interaction of Estrogen Depletion and Dietary Induced Metabolic Acidosis on Bone Time dependent Structural Properties: A Single Branch, Or a Bundle of Sticks?"

7. Les, C. M., C. L. Pechey, E. M. Michels, M. Fang, J. M. MacLeay, A. S. Turner, and M. M. Banaszak Holl. "Estrogen Depletion Is Associated with a Loss in Anatomic Variability in Both Bone Collagen D-spacing, and in Bone Material Pre-stress." Diss. N.d. Abstract. (n.d.): n. pag.

8. Wallace, Joseph M., Blake Erickson, Clifford M. Les, Bradford G. Orr, and Mark M. Banaszak Holl. "Distribution of Type I Collagen Morphologies in Bone: Relation to Estrogen Depletion." Bone 46.5 (2010): 1349-354.

9. Frasca, Peter. "Scanning-Electron Microscopy Studies of 'Ground Substance' in the Cement Lines, Resting Lines, Hypercalcified Rings and Reversal Lines of Human Cortical Bone." Cells Tissues Organs 109.2 (1981): 115-21.

10. $\quad$ Roylance, David. "Engineering Viscoelasticity." (n.d.): n. pag. Massachusetts Institute of Technology, 24 Oct. 2001. Web.

11. Katz, J.Lawrence. "Hard Tissue as a Composite Material-I. Bounds on the Elastic Behavior." Journal of Biomechanics 4.5 (1971): 455-73.

12. Jager, I., and P. Fratzel. "Mineralized Collagen Fibrils: A Mechanical Model with a Staggered Arrangement of Mineral Particles." Biophysical Journal 79.4 (2000): 1737-746.

13. Pidaparti, R.m.v., A. Chandran, Y. Takano, and C.h. Turner. "Bone Mineral Lies Mainly outside Collagen Fibrils: Predictions of a Composite Model for Osternal Bone." Journal of Biomechanics 29.7 (1996): 909-16.

14. Sasaki, Naoki, Tetsu Ikawa, and Akeharu Fukuda. "Orientation of Mineral in Bovine Bone and the Anisotropic Mechanical Properties of Plexiform Bone." Journal of Biomechanics 24.1 (1991): 57-61. 
15. Wagner, H.D., and S. Weiner. "On the Relationship between the Microstructure of Bone and Its Mechanical Stiffness." Journal of Biomechanics 25.11 (1992): 1311-320.

16. Katz, J.Lawrence. "Composite material models for cortical bone" In: Cowin (1981): 171-84.

17. Giraud-Guille, M. M. "Twisted Plywood Architecture of Collagen Fibrils in Human Compact Bone Osteons." Calcified Tissue International 42.3 (1988): 167-80.

18. Reilly, Donald T., and Albert H. Burstein. "The Elastic and Ultimate Properties of Compact Bone Tissue." Journal of Biomechanics 8.6 (1975): 393-405.

19. Ashman, R.B., S.C. Cowin, W.C. Van Buskirk, and J.C. Rice. "A Continuous Wave Technique for the Measurement of the Elastic Properties of Cortical Bone." Journal of Biomechanics 17.5 (1984): 349-61.

20. Ascenzi, Antonio, and Ermanno Bonucci. "The Tensile Properties of Single Osteons." The Anatomical Record 158.4 (1967): 375-86.

21. Lakes, Roderic. "Materials with Structural Hierarchy." Nature 361.6412 (1993): 511-15.

22. McElhaney, J. H. "Dynamic Response of Bone and Muscle Tissue." J. appl. Physiol. 21 (1966): 1231-36.

23. Currey, J. "Differences in the Tensile Strength of Bone of Different Histological Types."Journal of Anatomy 93.1 (1959): 87-95.

24. Fois, M., A. Lamure, M. J. Fauran, and C. Lacabanne. "Study of Human Cortical Bone and Demineralized Human Cortical Bone Viscoelasticity." Journal of Applied Polymer Science 79.14 (2001): 2527-533.

25. Wright, T.M., Hayes, W.C. "Tensile Testing of Bone Over a Wide Range of Strain Rates: Effects of Strain Rate, Microstructure and Density.” Medical and Biological Engineering 14 (1976): 67180 .

26. Currey, John D., James Foreman, Ira Laketi?, Julie Mitchell, David E. Pegg, and Gwendolen C. Reilly. "Effects of Ionizing Radiation on the Mechanical Properties of Human Bone." Journal of Orthopaedic Research 15.1 (1997): 111-17.

27. Danielsen, C.C., Li. Mosekilde, J. Bollerslev, and Le. Mosekilde. "Thermal Stability of Cortical Bone Collagen in Relation to Age in Normal Individuals and in Individuals with Osteopetrosis." Bone 15.1 (1994): 91-96.

28. Zioupos, P. "Ageing Human Bone: Factors Affecting Its Biomechanical Properties and the Role of Collagen." Journal of Biomaterials Applications 15 (2001): 187-229.

29. Zioupos, P., J. D. Currey, and A. J. Hamer. "The Role of Collagen in the Declining Mechanical Properties of Aging Human Cortical Bone." Journal of Biomedical Materials Research 45.2 (1999): 108-16. 
30. Wang, Zhongkui, and Shinichi Hirai. "Modeling and Parameter Identification of Rheological Object Based on FE Method and Nonlinear Optimization." Proc. IEEE/RSJ International Conference on Intelligent Robots and Systems (IROS '09) (2009): 1968-973.

31. Yamashita, Junro, Xiaoe Li, Benjamin R. Furman, H. Ralph Rawls, Xiaodu Wang, and C. Mauli Agrawal. "Collagen and Bone Viscoelasticity: A Dynamic Mechanical Analysis." Journal of Biomedical Materials Research 63.1 (2002): 31-36.

32. Siegmund, T., M. Allen, and D. Burr. "Failure of Mineralized Collagen Fibrils: Modeling the Role of Collagen Cross-linking." Journal of Biomechanics 41.7 (2008): 1427-435.

33. Ramaekers, J.G.M. "The Rheological Behaviour of Skeletal Material Originating From Several Classes of Vertebrates." Netherlands Journal of Zoology 29.2 (1978): 166-76.

34. Johnson, M, and J L Katz. "Some New Developments in the Rheology of Bone." Biorheology Suppl, 1 (1984): 169-174.

35. Currey, J. D. "Effects of Differences in Mineralization on the Mechanical Properties of Bone." Philosophical Transactions of the Royal Society B: Biological Sciences 304.1121 (1984): 509-18.

36. Gourrier, Aurelien, Wolfgang Wagermaier, Manfred Burghammer, Donna Lammie, Himadri S. Gupta, Peter Fratzl, Christian Riekel, Tim J. Wess, and Oskar Paris. "Scanning X-ray Imaging with Small-angle Scattering Contrast." Journal of Applied Crystallography 40.S1 (2007): S78-82.

37. Shultz, T. R., J. D. Blaha, T. A. Gruen, and T. L. Norman. "Cortical Bone Viscoelasticity and Fixation Strength of Press-Fit Femoral Stems: A Finite Element Model." Journal of Biomechanical Engineering 128.1 (2006): 7-12.

38. Kanis, J. "Diagnosis of Osteoporosis and Assessment of Fracture Risk." The Lancet359.9321 (2002): 1929-936.

39. Hodge, A. J., and J. A. Petruska. "Recent Studies with the Electron Microscope on Ordered Aggregates of the Tropocollagen Molecule." Aspects of Protein Structure. Ed. G. N. Ramachandran. New York: Academic Press, 1963. 269-300.

40. Weiner, S., and W. Traub. "Organization of Hydroxyapatite Crystals within Collagen Fibrils." FEBS Letters 206.2 (1986): 262-66.

41. De Micheli, P.o., and U. Witzel. "Microstructural Mechanical Study of a Transverse Osteon under Compressive Loading: The Role of Fiber Reinforcement and Explanation of Some Geometrical and Mechanical Microscopic Properties." Journal of Biomechanics 44.8 (2011): $1588-592$.

42. Les, C., et al. "Determinants of Ovine Compact Bone Viscoelastic Properties: Effects of Architecture, Mineralization, and Remodeling." Bone 35.3 (2004): 729-38.

43. Les, C. M., J. L. Vance, G. T. Christopherson, A. S. Turner, G. W. Divine, and D. P. Fyhrie. "Long-term Ovariectomy Decreases Ovine Compact Bone Viscoelasticity." Journal of Orthopaedic Research 23.4 (2005): 869-76.

44. Lakes, Roderic S. Viscoelastic Solids. Boca Raton: CRC, 1999. 
45. Piekarski, K., and M. Munro. "Transport Mechanism Operating between Blood Supply and Osteocytes in Long Bones." Nature 269.5623 (1977): 80-82.

46. Buechner, P.m., D. Stone, and R.s. Lakes. "Viscoelastic Behavior of Superplastic $37 \mathrm{Wt} \% \mathrm{~Pb} 63$ Wt\% Sn over a Wide Range of Frequency and Time." Scripta Materialia 41.5 (1999): 561-67.

47. Stephen, Weiner, and Traub Wolfie. "Bone Structure: From Angstroms to Microns." The FSAEB Journal 6 (1992): 879-85.

48. "Abaqus User Subroutines Reference Manual (6.10)." Sharcnet.ca. Dassault Systemes Simulia Corp., n.d. Web. 28 Jan. 2013.

49. Richter, Frank. Upsetting and Viscoelasticity of SiO2: Experiments, Interpretation and Simulation. Thesis. Technical University of Berlin, 2005. Berlin: n.p., 2006.

50. Shepherd, Tara, Ryan Roeder, Jingzhou Zhang, Glen Niebur, and Timothy Ovaert. "Direct Comparison of Nanoindentation and Macroscopic Measurements of Bone Viscoelasticity." Journal of the Mechanical Behavior of Biomedical Materials, 4.8 (2011): 2055-2062.

51. Pechey, C. L., E. M. Michels, M. Fang, J. M. MacLeay, M. M. Banaszak Holl, and C. M. Les. "Histologic and Mineralization Correlates of Estrogen Depleted-related Changes in Ovine Bone Collagen I D-Spacing." Diss. N.d. Abstract. (n.d.): n. pag.

52. "What Is Osteoporosis?" Iofbonehealth.org. International Osteoporosis Foundation, 2012. Web. 11 Feb. 2013.

53. Boyde, A. "Electron microscopy of the mineralizing front." Metabolic bone disease and related research (Suppl.): 69-78. 1980.

54. Landis, William J., Karen J. Hodgens, James Arena, Min Ja Song, and Bruce F. McEwen. "Structural Relations between Collagen and Mineral in Bone as Determined by High Voltage Electron Microscopic Tomography." Microscopy Research and Technique33.2 (1996): 192-202.

55. Miller, SC, L Saintgeorges, BM Bowman, WSS Jee, and INT SCANNING MICROSCOPY. "Bone Lining Cells - Structure and Function." Scanning Microscopy, 3.3 (1989): 953-961.

56. Sedlin, Elias D., and Carl Hirsch. "Factors Affecting the Determination of the Physical Properties of Femoral Cortical Bone." Acta Orthopaedica 37.1 (1966): 29-48.

57. Fang, Ming. "Type I Collagen Nanomorphology in Relation to Disease, Tissue Hierarchy, and Fibrillogenesis." Diss. University of Michigan, 2013.

58. Wallace, Joseph M., Qishui Chen, Ming Fang, Blake Erickson, Bradford G. Orr, and Mark M. Banaszak Holl. "Type I Collagen Exists as a Distribution of Nanoscale Morphologies in Teeth, Bones, and Tendons." Langmuir 26.10 (2010): 7349-354.

59. $\quad$ Ricardblum, S., and G. Ville. "Collagen Cross-linking." International Journal of Biochemistry 21.11 (1989): 1185-189.

60. Knott, L. "Collagen Cross-links in Mineralizing Tissues: A Review of Their Chemistry, Function, and Clinical Relevance." Bone 22.3 (1998): 181-87. 
61. Bayraktar, Harun H., Elise F. Morgan, Glen L. Niebur, Grayson E. Morris, Eric K. Wong, and Tony M. Keaveny. "Comparison of the Elastic and Yield Properties of Human Femoral Trabecular and Cortical Bone Tissue." Journal of Biomechanics 37.1 (2004): 27-35.

62. Quereda, F., N. Mendoza, M. Olalla, F. Baro, and M. Duran. "Prophylactic Approach for Asymptomatic Post-menopausal Women: Osteoporosis." Maturitas 52 (2005): 38-45.

63. D'Amelio, Patrizia, Anastasia Grimaldi, Stefania Di Bella, Stefano Z.M. Brianza, Maria Angela Cristofaro, Cristina Tamone, Giuliana Giribaldi, Daniela Ulliers, Gian P. Pescarmona, and Giancarlo Isaia. "Estrogen Deficiency Increases Osteoclastogenesis Up-regulating T Cells Activity: A Key Mechanism in Osteoporosis." Bone 43.1 (2008): 92-100.

64. Lindsay, Robert. "The Menopause and Osteoporosis." Obstetrics and Gynecology 87.2 (1996): 16S-9S.

65. Albright, Fuller, Patricia H. Smith, and Anna M. Richardson. "Postmenopausal Osteoporosis."The Journal of the American Medical Association 116.22 (1941): 2465-474.

66. Allen, Matthew R. "Skeletal Accumulation of Bisphosphonates: Implications for Osteoporosis Treatment." Expert Opinion on Drug Metabolism \& Toxicology 4.11 (2008): 1371-378.

67. Keam, Susan J., and Greg L. Plosker. "Prevention and Treatment of Osteoporosis in Postmenopausal Women." Disease Management \& Health Outcomes 12.1 (2004): 19-37.

68. Scotland, Graham, Norman Waugh, Pamela Royle, Paul McNamee, Rob Henderson, and Rosemary Hollick. "Denosumab for the Prevention of Osteoporotic Fractures in Post-Menopausal Women." PharmacoEconomics (2011): 1.

69. Palacios, Santiago, Mark Brincat, Tamer Erel, Marco Gambaciani, Irene Lambrinoudaki, Mette H. Moen, Karin Schenck-Gustafsson, Florence Tremollieres, Svetlana Vujovic, Margaret Rees, and Serge Rozenberg. "EMAS Clinical Guide: Selective Estrogen Receptor Modulators for Postmenopausal Osteoporosis." Maturitas 71 (2012): 194-98.

70. Blick, Stephanie K A., Sohita Dhillon, and Susan J. Keam. "Teriparatide." Drugs 68.18 (2008): 2709-737.

71. Oheim, Ralf, Michael Amling, Anita Ignatius, and Pia Pogoda. "Large Animal Model for Osteoporosis in Humans: The Ewe." European Cells and Materials 24 (2012): 372-85.

72. Hillier, Maria L., and Lynne S. Bell. "Differentiating Human Bone from Animal Bone: A Review of Histological Methods." Journal of Forensic Sciences 52.2 (2007): 249-63.

73. Zarrinkalam, M. R., H. Beard, C. G. Schultz, and R. J. Moore. "Validation of the Sheep as a Large Animal Model for the Study of Vertebral Osteoporosis." European Spine Journal 18 (2009): 244-53.

74. Kaveh, Kamran, Mohd Zuki AbuBakar, and Tengku Asmi Ibrahim. "Osteoporosis Induction in Animal Model." American Journal of Animal and Veterinary Sciences 5.2 (2010): 139-45.

75. Lanyon, L. E., and D. G. Baggott. "Mechanical Function as an Influence on the Structure and Form of Bone." The Journal of Bone and Joint Surgery 58 (1976): 436-43. 
76. Newman, E., A. Turner, and J. Wark. "The Potential of Sheep for the Study of Osteopenia: Current Status and Comparison with Other Animal Models." Bone 16.4 (1995): S277-284.

77. Leslie, William D., and John T. Schousboe. "A Review of Osteoporosis Diagnosis and Treatment Options in New and Recently Updated Guidelines on Case Finding Around the World." Current Osteoporosis Reports 9 (2011): 129-40.

78. Currey, J. "The Effects of Strain Rate, Reconstruction and Mineral Content on Some Mechanical Properties of Bovine Bone." Journal of Biomechanics 8.1 (1975): 81-82.

79. $\quad$ Ricard-Blum, Sylvie. "The Collagen Family." Cold Spring Harbor Perspectives in Biology(2011): n. pag. 


\section{APPENDICES}

Appendix A: Experimental Ovine Data

\begin{tabular}{|c|c|c|c|c|c|c|c|c|}
\hline \multirow[b]{2}{*}{ Sheep } & \multirow[b]{2}{*}{ Specimen } & \multirow[b]{2}{*}{ Treatment } & \multirow[b]{2}{*}{ Sector } & \multirow[b]{2}{*}{ Side } & \multicolumn{4}{|c|}{ DMA Data (Tangent Delta_Frequency) } \\
\hline & & & & & TanD_1 & TanD_3 & TanD_9 & TanD_15 \\
\hline 22 & $\mathrm{C} 2222$ & Control & 5 & $\mathrm{Cd}$ & 0.098628 & 0.085794 & 0.060804 & 0.036278 \\
\hline 22 & C2219 & Control & 6 & $\mathrm{Cd}$ & 0.068954 & 0.060823 & 0.04108 & 0.022938 \\
\hline 11 & C1114 & Control & 6 & $\mathrm{Cd}$ & 0.085255 & 0.071874 & 0.04505 & 0.018304 \\
\hline 11 & C1111 & Control & 5 & $\mathrm{Cd}$ & 0.081187 & 0.062383 & 0.033776 & 0.009397 \\
\hline 6 & $\mathrm{C} 0615$ & Control & 5 & $\mathrm{Cd}$ & 0.09144 & 0.077092 & 0.05042 & 0.025226 \\
\hline 6 & $\mathrm{C} 0613$ & Control & 6 & $\mathrm{Cd}$ & 0.08666 & 0.073777 & 0.049291 & 0.028045 \\
\hline 22 & $\mathrm{C} 2215$ & Control & 2 & $\mathrm{Cr}$ & 0.068225 & 0.055921 & 0.031804 & 0.012429 \\
\hline 22 & C2207 & Control & 1 & $\mathrm{Cr}$ & 0.07035 & 0.060794 & 0.03861 & 0.020517 \\
\hline 11 & C1120 & Control & 2 & $\mathrm{Cr}$ & 0.09284 & 0.07164 & 0.035124 & 0.007162 \\
\hline 11 & C1108 & Control & 1 & $\mathrm{Cr}$ & 0.07276 & 0.063211 & 0.040497 & 0.021065 \\
\hline 6 & C0608 & Control & 2 & $\mathrm{Cr}$ & 0.07762 & 0.066401 & 0.045583 & 0.026755 \\
\hline 6 & C0603 & Control & 1 & $\mathrm{Cr}$ & 0.077544 & 0.068259 & 0.045227 & 0.024657 \\
\hline 18 & C1818 & OVX & 6 & $\mathrm{Cd}$ & 0.068283 & 0.056258 & 0.029432 & 0.005464 \\
\hline 18 & C1802 & OVX & 5 & $\mathrm{Cd}$ & 0.06853 & 0.05673 & 0.032557 & 0.009208 \\
\hline 8 & C0820 & OVX & 5 & $\mathrm{Cd}$ & 0.093627 & 0.082971 & 0.056048 & 0.026157 \\
\hline 8 & C0808 & ovX & 6 & $\mathrm{Cd}$ & 0.076918 & 0.066762 & 0.044374 & 0.021738 \\
\hline 5 & C0518 & OVX & 6 & $\mathrm{Cd}$ & 0.081801 & 0.06645 & 0.04365 & 0.022011 \\
\hline 5 & C0512 & OVX & 5 & $\mathrm{Cd}$ & 0.086054 & 0.068178 & 0.039556 & 0.015972 \\
\hline 18 & C1811 & OVX & 2 & $\mathrm{Cr}$ & 0.070244 & 0.058924 & 0.034877 & 0.012325 \\
\hline 18 & C1809 & OVX & 1 & $\mathrm{Cr}$ & 0.075466 & 0.062502 & 0.036316 & 0.012177 \\
\hline 8 & $\mathrm{C} 0816$ & OVX & 1 & $\mathrm{Cr}$ & 0.066467 & 0.055985 & 0.035628 & 0.014889 \\
\hline 8 & C0802 & OVX & 2 & $\mathrm{Cr}$ & 0.073447 & 0.066112 & 0.048028 & 0.029362 \\
\hline 5 & $\mathrm{C} 0514$ & ovX & 2 & $\mathrm{Cr}$ & 0.076028 & 0.064128 & 0.037049 & 0.013405 \\
\hline 5 & C0503 & ovx & 1 & $\mathrm{Cr}$ & 0.076469 & 0.065369 & 0.041262 & 0.0176 \\
\hline
\end{tabular}

\section{Appendix B: Bending Calculations: Composite Stress Estimation}

The three point bending tests on the ovine radius/ulna bone were executed on an apparatus with

outer supports separated by a $15 \mathrm{~mm}$ distance. The loading conditions for each test are composed of a static load of $550 \mathrm{mN}$ and a dynamic load of $500 \mathrm{mN}$. The bone samples themselves were $1.75 \mathrm{~mm} \times 1.75$

$\mathrm{mm} \times 19 \mathrm{~mm}$ since they were machined from the whole bone. Using this information, the stresses imparted on the bone sections can be estimated. First, the moment of inertia is approximated. 


$$
I=\frac{1}{12} b h^{3}=\frac{1}{12}(1.75 \mathrm{~mm})(1.75 \mathrm{~mm})^{3}=7.816 E-13 \mathrm{~m}^{4}
$$

The dynamic loading conditions mean that the bending moments applied to the bone sections are dynamic as well. Here, three bending moments are calculated based on the static, maximum, and minimum loads. Shear and bending moment diagrams were used to estimate the moments (not shown).

$$
\begin{gathered}
M_{\text {static }}=(7.5 \mathrm{~mm})(275 \mathrm{Nm})=2.063 \mathrm{mNm} \\
M_{\text {maximum }}=(7.5 \mathrm{~mm})(400 \mathrm{Nm})=3 \mathrm{mNm} \\
M_{\text {minimum }}=(7.5 \mathrm{~mm})(150 \mathrm{Nm})=1.125 \mathrm{mNm}
\end{gathered}
$$

Then, the use of beam theory provides insight into the stresses themselves.

$$
\begin{gathered}
\sigma_{\text {static }}= \pm \frac{M y}{I}= \pm \frac{(2.063 \mathrm{mNm})\left(\frac{1.75 \mathrm{~m}}{2}\right)}{7.816 E-13 \mathrm{~m}^{4}}= \pm 2.31 \mathrm{MPa} \\
\sigma_{\text {maximum }}= \pm \frac{(3 \mathrm{mNm})\left(\frac{1.75 \mathrm{~m}}{2}\right)}{7.816 \mathrm{E}-13 \mathrm{~m}^{4}}= \pm 3.36 \mathrm{MPa} \\
\sigma_{\text {minimum }}= \pm \frac{(3 \mathrm{mNm})\left(\frac{1.75 \mathrm{~m}}{2}\right)}{7.816 \mathrm{E}-13 \mathrm{~m}^{4}}= \pm 1.26 \mathrm{MPa}
\end{gathered}
$$

The three calculated bending stresses are fundamental to the application of sinusoidal loading conditions on the model. The amplitude feature in Abaqus provides a simple approach to mimic these loading conditions.

\section{Appendix C: Protocol to Edit the Input File}

The Abaqus user interface provides a simple-visual approach to develop finite element models; however, understanding how to edit and utilize the input file can produce models with even greater complexity while also reducing the time it takes to develop them. Some of the objectives of this particular composite model involved modifying the number of steps and loading condition behaviors, both of which could be accomplished with quick alterations to specific variables located within the input file. The primary tool used to edit the input files was a "find and replace" function that can be found in many 
document programs. The particular program used in this instance was the Text Editor program. This section will highlight two primary changes to the input file that were applied to decrease the time it takes to modify the model.

One of the goals of the analysis was to gather enough data to make the curve fitting as accurate as possible. It was determined that at least twenty data points per cycle would yield an accurate measurement of the viscoelastic measurement, tangent delta. The simulation caused the composite structure to be loaded for twenty cycles for each analysis; thus 400 data points were required to meet the twenty data points per cycle goal. The python script gathered a single data point at the end of each step, so a total of 400 steps were necessary. Creating 400 steps through the gui is very time consuming as multiple actions are required to create a single step; however, multiple steps can be created through the input file by simple uses of copy, paste, and the find and replace function. The steps in the input file have the following format:

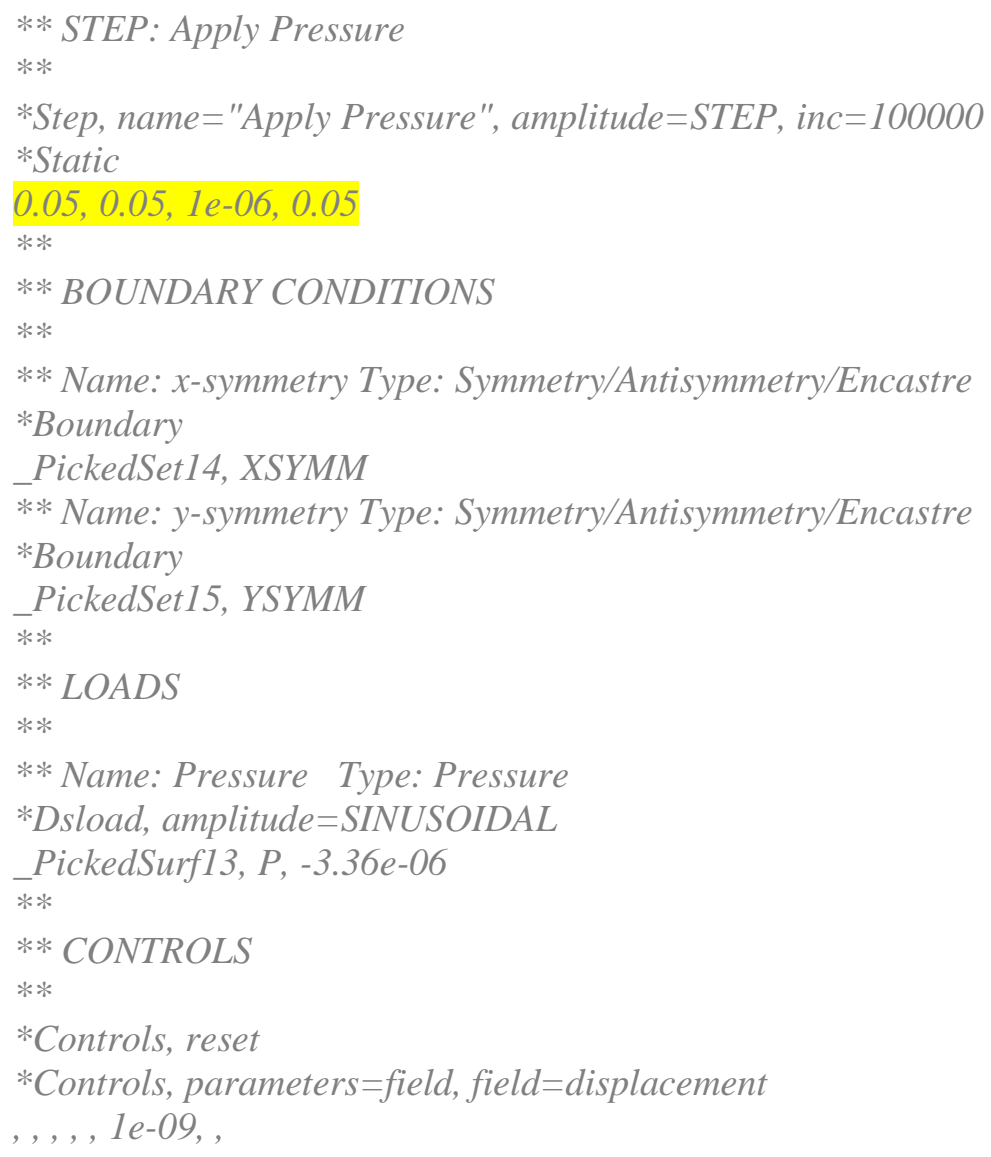




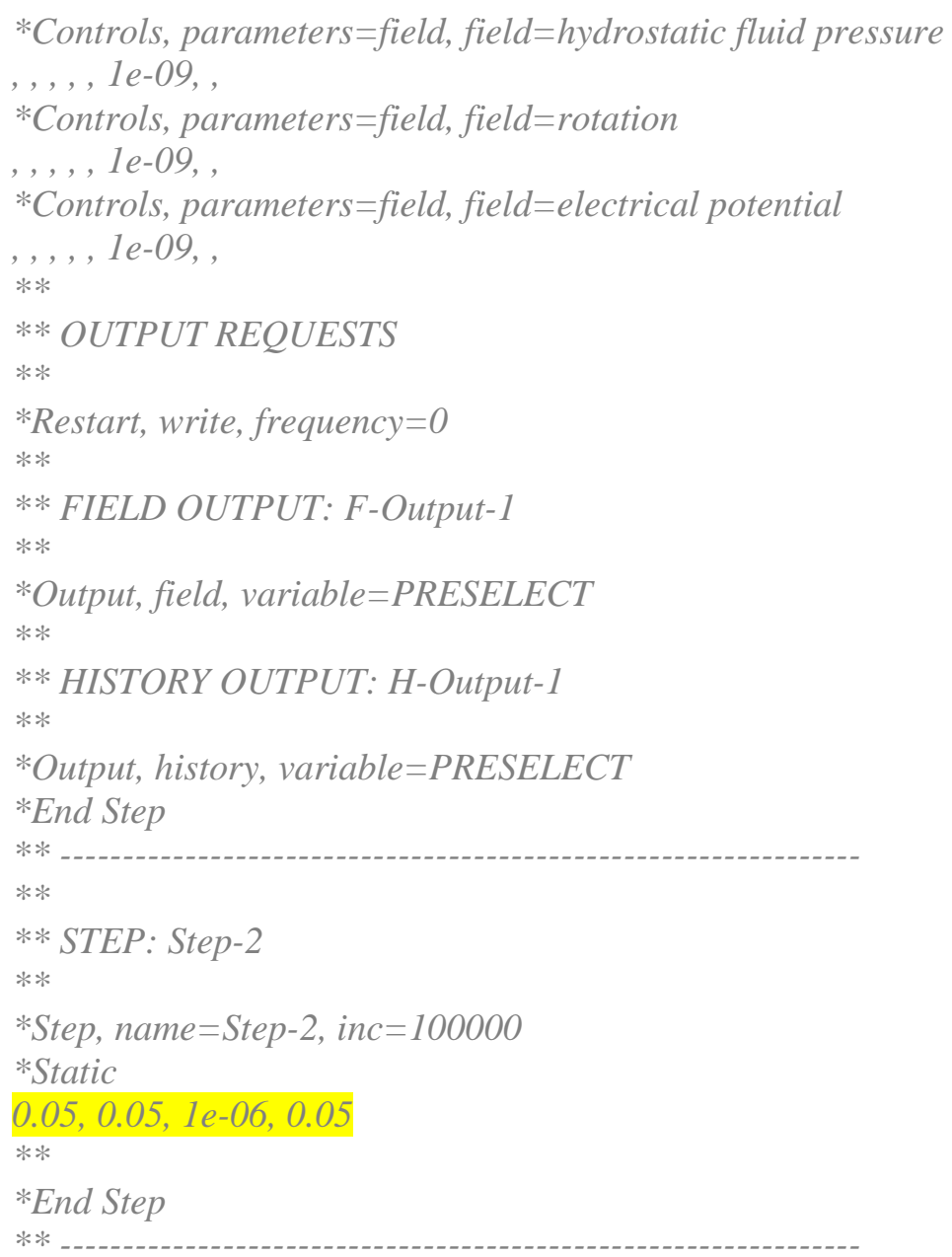

The boundary conditions, loads, output requests, and controls are only required in the first step, so subsequent steps will be identical to Step-2. This means that Step-2 can be copied and pasted to create the remaining 398 steps. The first 100 steps were created in this fashion by merely altering the name of the step to coincide with the number. The next 300 steps could be created by using find and replace to modify the hundreds place of the first 100 steps. Using this method to create large numbers of steps is very quick and useful.

Another goal for the model involved altering the frequency of the applied load so that the same composite structure could be loaded at various frequencies. The amplitude feature in Abaqus made this adjustment very simple. The angular frequency $(\omega=2 \pi f)$ is represented as a single value in the input file: 


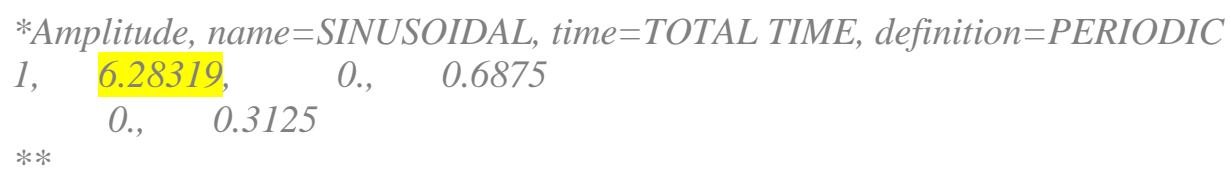

This section is located right after the assembly and right before the materials code in the input file. The highlighted number is the angular frequency at a $1 \mathrm{~Hz}$ frequency. By simply changing this number, the frequency of the sinusoidal loading conditions also changes. Any modifications to the frequency would also change the time it takes to complete 20 cycles, so the step time would also have to be adjusted. A series of numbers was highlighted in the previous code that revealed the layout of the steps. The numbers $(0.05,0.05,1 \mathrm{e}-06,0.05)$ represent the step time, initial increment, smallest increment, and largest increment in that order. These numbers are also the values at the $1 \mathrm{~Hz}$ frequency. As long as the frequency is known, the time it takes for a single cycle to transpire can be determined. This number would have to be divided by the number of steps per cycle, 20 , in order to change the step time to the appropriate amount. The find and replace function can adjust the entire 400 steps of the input file in a single action.

\section{Appendix D: Abaqus Warnings and Errors}

This section highlights some of the major warnings and errors that were encountered in the development of the composite model and how these issues were resolved. Abaqus forums, such as iMechanica and ENG-TIPS, and the Abaqus documentation served as primary sources of information to resolve many of the issues that were experienced in the development of the composite model.

\begin{tabular}{|l|l|}
\hline Warnings and Errors & Solutions \\
\hline Zero forces everywhere & $\begin{array}{l}\text { Abaqus contains a pre-set value for what magnitude of loading is } \\
\text { considered small enough to be zero. Due to the magnitude of the } \\
\text { units used, the pressure entered caused small nodal forces to be } \\
\text { ignored. The pre-set value for zero forces was adjusted to resolve } \\
\text { the warning. }\end{array}$ \\
\hline $\begin{array}{l}\text { Displacement increment for } \\
\text { contact too big }\end{array}$ & $\begin{array}{l}\text { Originally, the upper portion of the model that contains the HA and } \\
\text { smaller collagen section were two different instances. Since the } \\
\text { model was pulled apart in tension, the instances would displace too } \\
\text { quickly for Abaqus to understand. This error was resolved by } \\
\text { making them a single instance (perfectly bonded HA and collagen) } \\
\text { by utilizing the partition feature in Abaqus. }\end{array}$ \\
\hline $\begin{array}{l}\text { There are } 3 \text { unconnected regions in } \\
\text { the model }\end{array}$ & $\begin{array}{l}\text { Simply put, the interactions were not in place before the job was } \\
\text { run. }\end{array}$ \\
\hline $\begin{array}{l}\text { Time increment required is less } \\
\text { than minimum }\end{array}$ & $\begin{array}{l}\text { Abaqus automatically requires a minimum time increment and this } \\
\text { value simply had to be adjusted to allow contact analysis to take }\end{array}$ \\
\hline
\end{tabular}




\begin{tabular}{|c|c|}
\hline & place. \\
\hline $\begin{array}{l}\text { Too many attempts made for this } \\
\text { increment }\end{array}$ & $\begin{array}{l}\text { The analysis was not properly constrained. I believe this issue } \\
\text { occurred because of the error discussed in the previous solution for } \\
\text { the large displacement increment. Once the HA part and collagen } \\
\text { part were made into one, the error was fixed. }\end{array}$ \\
\hline $\begin{array}{l}\text { For *tie pair }(), \text { adjusted nodes } \\
\text { with very small adjustments were } \\
\text { not printed. }\end{array}$ & $\begin{array}{l}\text { Tie constraints adjust node displacements to maintain the contact } \\
\text { between two surfaces. In this case, the adjustments that fall below } \\
\text { some threshold value are not shown in the results, but are still used } \\
\text { in the analysis. }\end{array}$ \\
\hline $\begin{array}{l}\text { User subroutine will be used with } \\
\text { the STATEV array dimensioned to } \\
\text { zero since the depvar option is not } \\
\text { used with this material. } \\
\text { Consequently, defining STATEV } \\
\text { entries in subroutine UMAT will } \\
\text { cause code execution errors. }\end{array}$ & $\begin{array}{l}\text { The material definition for collagen required an input for the } \\
\text { number of solution dependent variables used in the SDVINI user } \\
\text { subroutine. This input could be entered in the depvar option in the } \\
\text { material definition in abaqus and would allow SDVINI to be used } \\
\text { in conjunction with the UMAT. }\end{array}$ \\
\hline $\begin{array}{l}2 \text { nodes have dof on which } \\
\text { incorrect boundary conditions may } \\
\text { have been specified. }\end{array}$ & $\begin{array}{l}\text { The tie constraints and boundary conditions overlap at two } \\
\text { locations causing this warning. There is no significant alteration to } \\
\text { the results as a consequence of this warning. }\end{array}$ \\
\hline Floating point error & $\begin{array}{l}\text { This error often occurs when a value is divided by zero somewhere } \\
\text { in the user subroutine code. In this case, one of the user defined } \\
\text { constants was inadvertently left out. }\end{array}$ \\
\hline $\begin{array}{l}\text { Too many attempts made for this } \\
\text { increment: } \\
\text {-Force equilibrium not achieved } \\
\text { within tolerance } \\
\text {-The rate of convergence is very } \\
\text { slow. Convergence is judged } \\
\text { unlikely }\end{array}$ & $\begin{array}{l}\text { Likely due to inadequate mesh size, undefined contact interactions, } \\
\text { incorrect Jacobian definition (UMAT), or large time increment size. } \\
\text { In this case, a syntax error within the UMAT code caused the issue. } \\
\text { Write statements within the user subroutine provided hints to the } \\
\text { location of the error. }\end{array}$ \\
\hline $\begin{array}{l}\text { Abaqus detected lock file .lck. } \\
\text { please confirm that no other } \\
\text { applications are attempting to write } \\
\text { to the output database associated } \\
\text { with this job before removing the } \\
\text { lock file and resubmitting. }\end{array}$ & $\begin{array}{l}\text { Abaqus automatically creates a .lck file for the .odb file that is } \\
\text { being accessed from the Python Development Environment (PDE). } \\
\text { This .lck file prevents the user from altering the .odb file while it is } \\
\text { being accessed. This error occurred when Abaqus crashed, leaving } \\
\text { the .lck file active even though the PDE wasn't in use. To resolve } \\
\text { the issue, the .lck file was simply deleted. }\end{array}$ \\
\hline $\begin{array}{l}8 \text { elements are distorted. Either the } \\
\text { isoparametric angles are out of the } \\
\text { suggested limits or the triangular or } \\
\text { tetrahedral quality measure is bad. } \\
\text { The elements have been identified } \\
\text { in element set WarnElemDistorted. }\end{array}$ & $\begin{array}{l}\text { This warning occurred specifically because of the coarse mesh that } \\
\text { was used on the composite model. Abaqus forced the elements to } \\
\text { be distorted in order to account for the partitions. This warning was } \\
\text { removed by reducing the mesh seed size and thus creating more } \\
\text { nodes and elements. }\end{array}$ \\
\hline
\end{tabular}

\section{Appendix E: User Subroutine Code}

$\mathrm{C}$

C SDVINI SUBROUTINE TO INITIALIZE AND KEEP TRACK OF STRESS INCREMENTS

C FROM THE PREVIOUS CALCULATION

$\mathrm{C}$

SUBROUTINE SDVINI(STATEV,COORDS,NSTATV,NCRDS,NOEL,NPT, 1 LAYER,KSPT) 


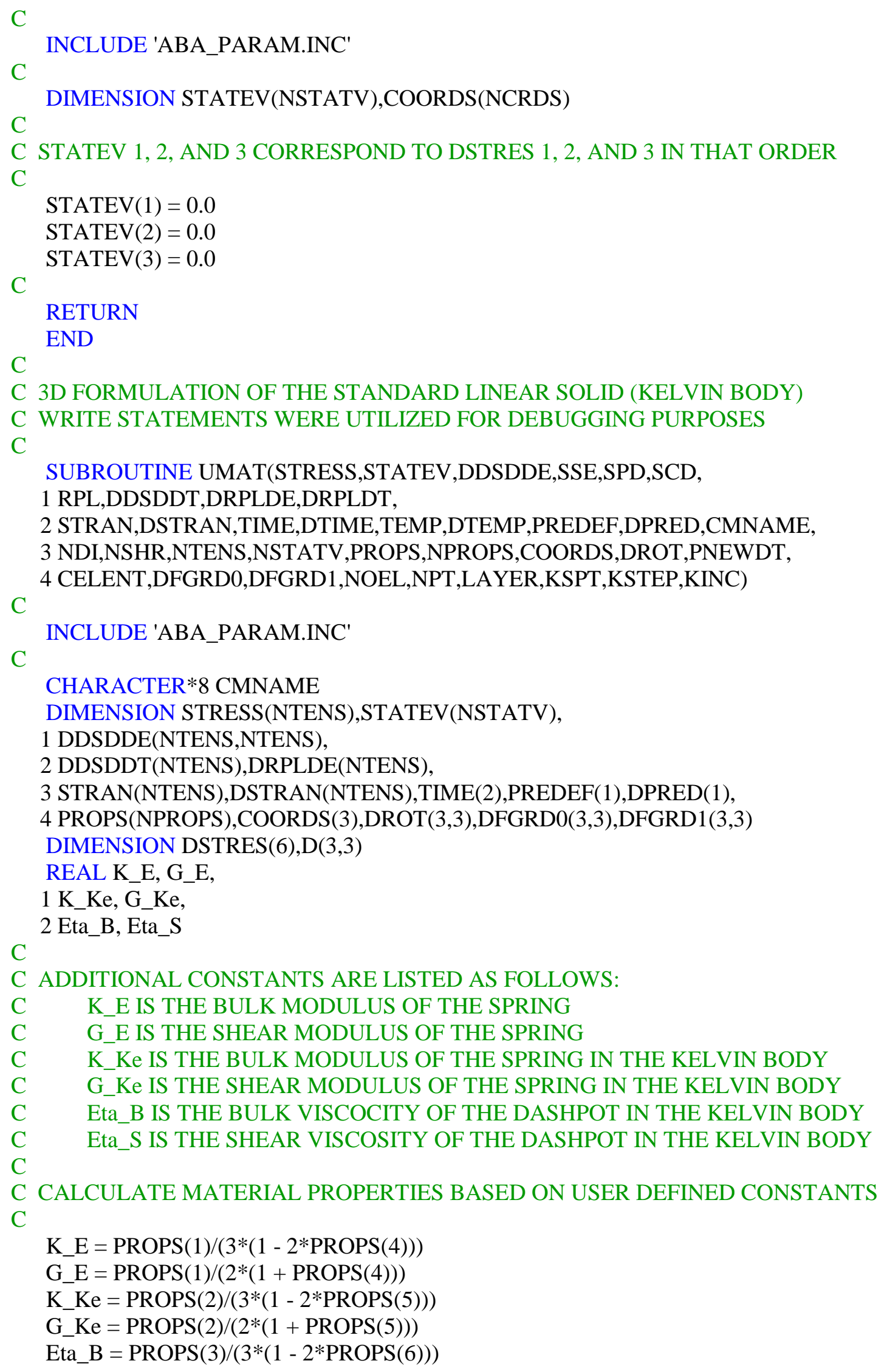




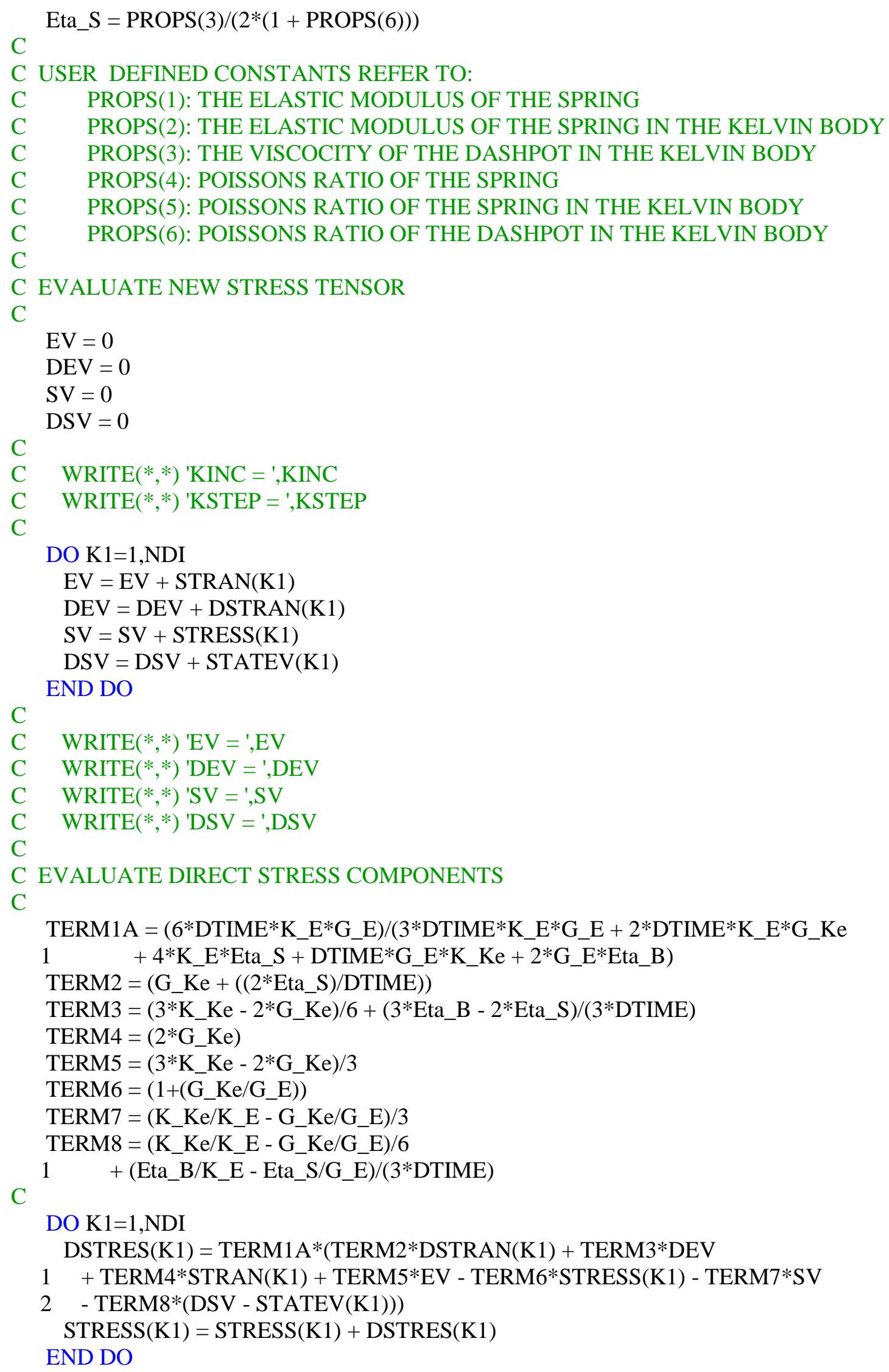




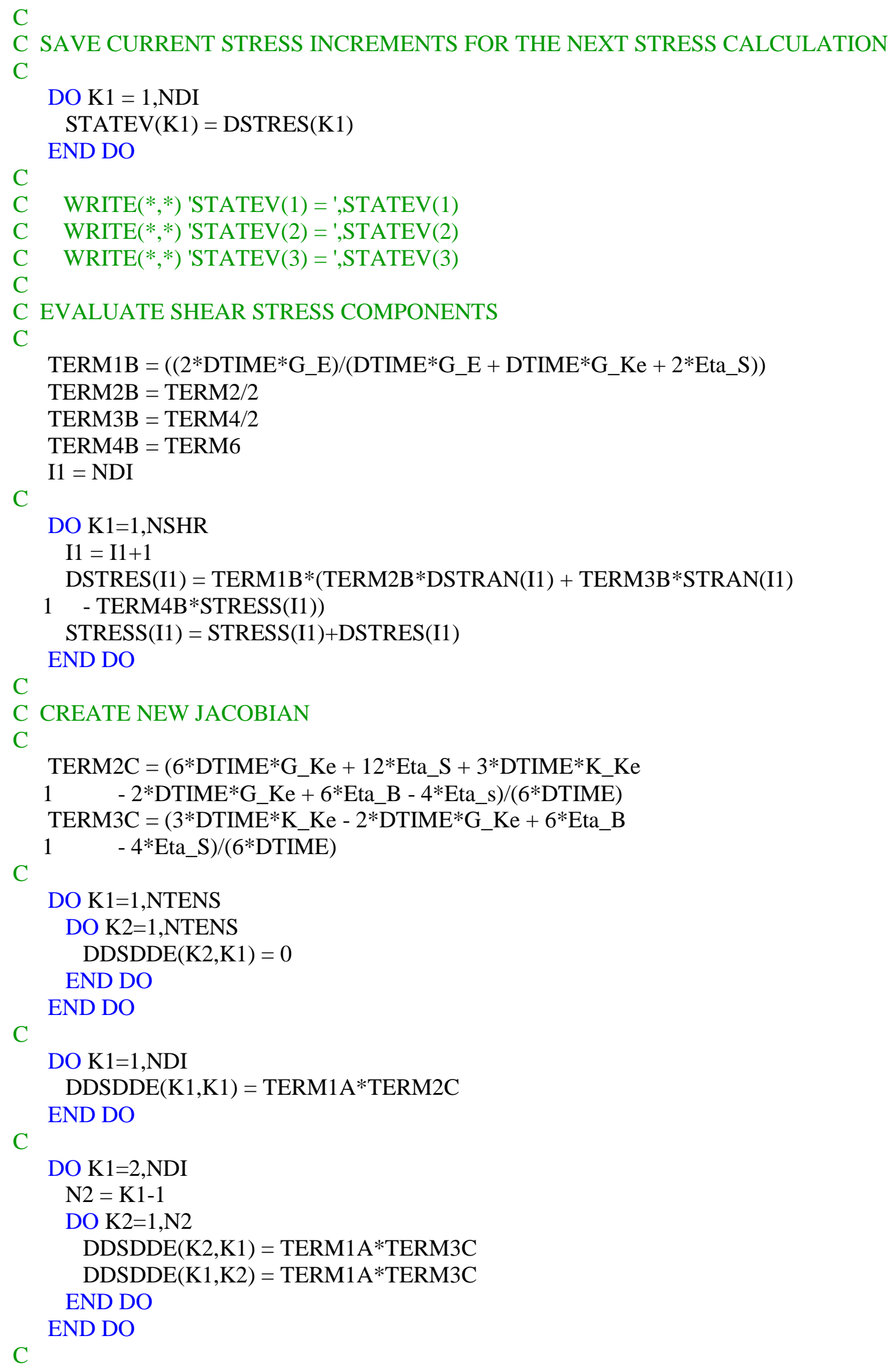




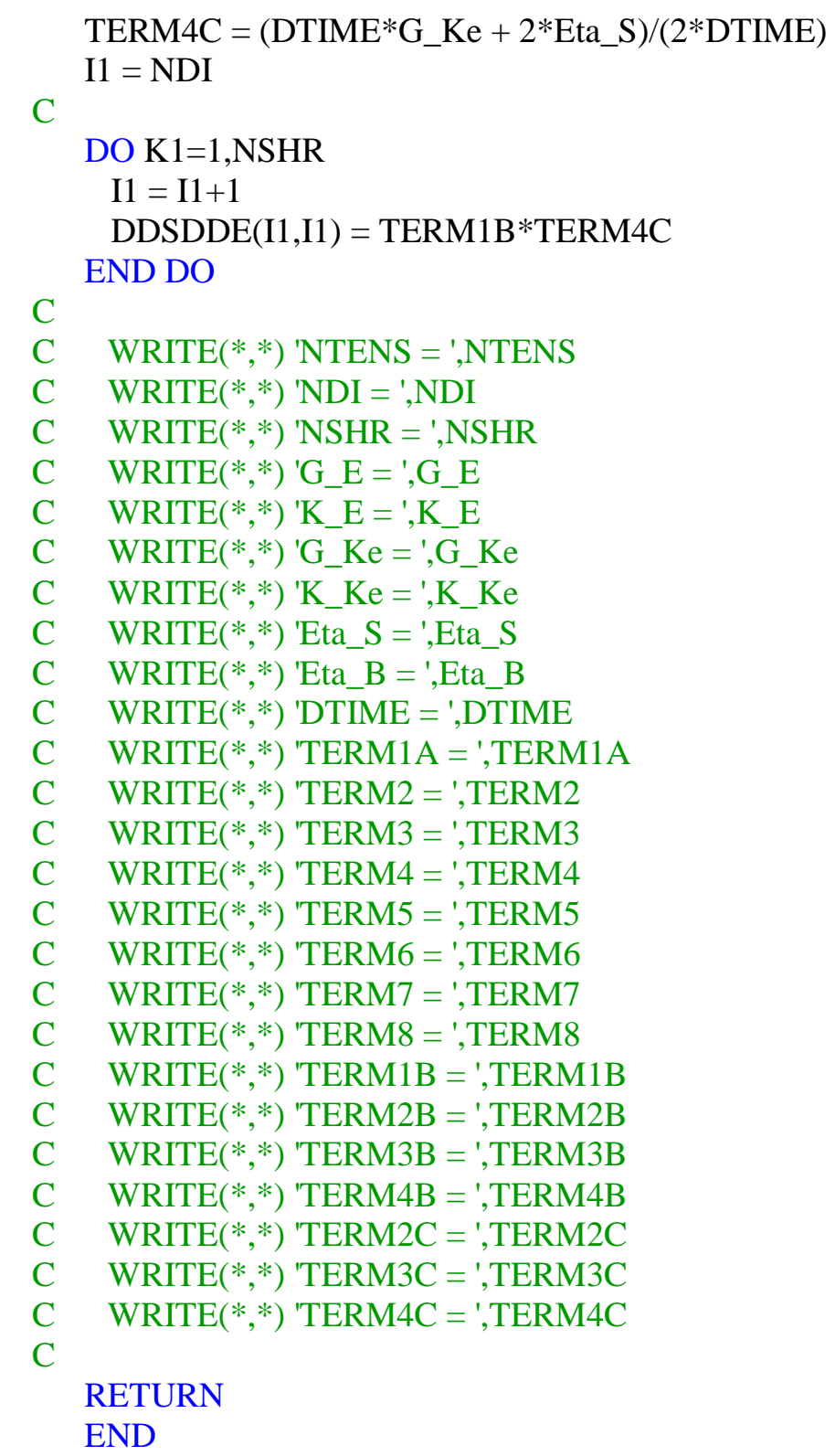

\section{Appendix F: Linear Viscoelastic Calculations}

The hand calculations are based on the Kelvin-Voigt body form of the standard linear solid. This section will show one method used to analyze the stress relaxation and creep behaviors of rheological models. 


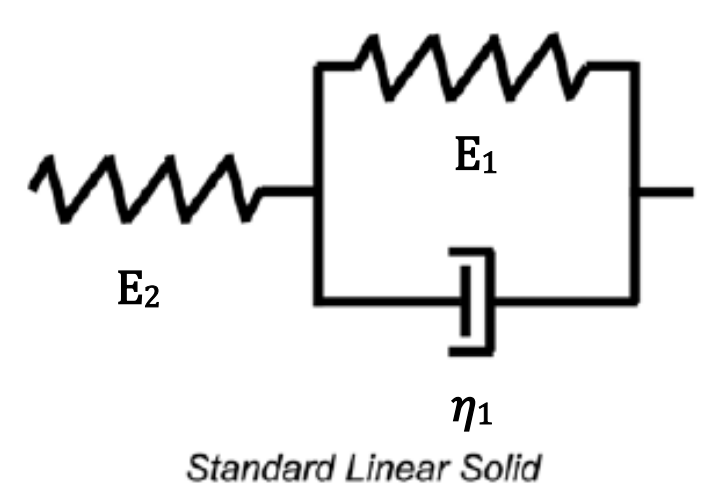

The governing equation of this rheological model is:

$$
\sigma+\frac{E_{1}+E_{2}}{\eta_{1}} \dot{\sigma}=\frac{\eta_{1}}{1+\frac{E_{1}}{E_{2}}} \dot{\varepsilon}+\frac{1}{\frac{1}{E_{1}}+\frac{1}{E_{2}}} \varepsilon
$$

Stress Relaxation:

Stress relaxation refers to the behavior that occurs when the material is strained to a specific amount and held there. The material initially stresses in response to this deflection but the stress decreases over time. The initial conditions, $\varepsilon(0)=\varepsilon_{o}$ and $\dot{\varepsilon}=0$, reduce the governing equation to:

$$
\dot{\sigma}+\frac{E_{1}+E_{2}}{\eta_{1}} \sigma=\frac{E_{1} E_{2}}{\eta_{1}} \varepsilon_{o}
$$

Calculating the following integrating factor yields:

$$
\mu(t)=e^{\int \frac{E_{1}+E_{2}}{\eta_{1}} d t}=e^{\frac{E_{1}+E_{2}}{\eta_{1}} t}=e^{\frac{t}{\tau}}
$$

where $\tau$ is the time constant and equal to $\frac{E_{1}+E_{2}}{\eta_{1}}$. Using the integrating factor: 


$$
\frac{\sigma}{\varepsilon_{o}}=\frac{\int e^{\frac{t}{\tau}}\left[\frac{E_{1} E_{2}}{\eta_{1}}\right] d t}{e^{\frac{t}{\tau}}}=\frac{e^{\frac{t}{\tau}\left[\frac{E_{1} E_{2}}{\eta_{1}}\right] \tau+C}}{e^{\frac{t}{\tau}}}=\frac{1}{\frac{1}{E_{1}}+\frac{1}{E_{2}}}+C e^{-\frac{t}{\tau}}
$$

With the boundary condition, $\sigma(0)=E_{2} \varepsilon_{o}$, the final stress relaxation equation becomes:

$$
\sigma=\left[\frac{E_{1} E_{2}}{E_{1}+E_{2}}+\frac{E_{2}^{2}}{E_{1}+E_{2}} e^{-\frac{t}{\tau}}\right] \varepsilon_{o}
$$

Creep:

When a constant stress is applied to a material and it deflects, not only initially, but also gradually over time, the material is said to have experienced creep. The initial conditions, $\sigma(0)=\sigma_{o}$ and $\dot{\sigma}=0$, alter the governing equation:

$$
\dot{\varepsilon}+\frac{1+\frac{E_{1}}{E_{2}}}{\eta_{1}\left(\frac{1}{E_{1}}+\frac{1}{E_{2}}\right)} \varepsilon=\frac{1+\frac{E_{1}}{E_{2}}}{\eta_{1}} \sigma_{o}
$$

Once again, an integrating factor must be determined.

$$
\mu(t)=e^{\int \frac{1+\frac{E_{1}}{E_{2}}}{\eta_{1}\left(\frac{1}{E_{1}}+\frac{1}{E_{2}}\right)} d t}=e^{\frac{\frac{E_{2}+E_{1}}{E_{2}}}{\eta_{1}\left(\frac{E_{1}+E_{2}}{E_{1} E_{2}}\right)} t}=e^{\frac{E_{1}}{\eta_{1}} t}=e^{\frac{t}{\tau}}
$$

Where the time constant, $\tau$, is $\frac{\eta_{1}}{E_{1}}$. Utilizing the integration factor:

$$
\frac{\varepsilon}{\sigma_{o}}=\frac{\int e^{\frac{t}{\tau}\left[\frac{1+\frac{E_{1}}{E_{2}}}{\eta_{1}}\right] d t}}{e^{\frac{t}{\tau}}}=\frac{e^{\frac{t}{\tau}\left[\frac{1+\frac{E_{1}}{E_{2}}}{\eta_{1}}\right] \tau+C}}{e^{\frac{t}{\tau}}}=\frac{1}{\frac{1}{E_{1}}+\frac{1}{E_{2}}}+C e^{-\frac{t}{\tau}}
$$

With the boundary condition, $\varepsilon(0)=\frac{\sigma_{o}}{E_{2}}$, the creep equation becomes: 


$$
\varepsilon=\left[\frac{E_{1} E_{2}}{E_{1}+E_{2}}-\frac{1}{E_{1}} e^{-\frac{t}{\tau}}\right] \sigma_{o}
$$

Both the creep and stress relaxation behaviors are exponential expressions with differing time constants and similar relaxation moduli. The relaxation modulus of each system refers to the modulus of elasticity composed of the spring network after the system has undergone either creep or stress relaxation for a sufficiently long time $(t \approx \infty)$.

\section{Appendix G: Python Script}

This data acquisition code could be used for every model. By simply commenting and uncommenting the odb file name and respective node list, the code can be used to acquire the data from the model of choice.

\# Python script to write displacements for desired nodes into separate \# files. Each file contains the displacements in the $\mathrm{x}$-direction from \# the last increment of every step.

\# Import odb commands from odbAccess import * from abaqusConstants import * \# Import serialization commands import pickle \# Import OS commands import os

\# Open odb file odb $=$ openOdb('/home/mimendoz/NormalDSpacing.odb')

$\#$ odb $=$ openOdb('/home/mimendoz/LowDSpacing.odb')

$\#$ odb $=$ openOdb('/home/mimendoz/HighDSpacing.odb')

\#odb $=$ openOdb('/home/mimendoz/LowDSpacingConstMin.odb')

\#odb $=$ openOdb('/home/mimendoz/HighDSpacingConstMin.odb')

$\#$ odb $=$ openOdb('/home/mimendoz/LowDSpacingConstCol.odb')

$\#$ odb $=$ openOdb('/home/mimendoz/HighDSpacingConstCol.odb')

\# Create folder for node displacement output (may not work on Windows)

if not os.path.exists('./node_displacement'): os.mkdir('./node_displacement')

\# Create array with all steps and count the number of steps.

step_list $=$ odb.steps.keys ()

numSteps $=$ len(step_list)

last_step $=$ odb.steps.keys ()$[-1]$ 
\# Define which nodes to extract displacements from

\# NormalDSpacing model

node_list $=[2,3,5,8,34,35,358,611,612,1422,1471,1520,1525,1972,3888,3890,3891,1]$

\# LowDSpacing model

\#node_list = [2,3,5,8,32,33,328,559,560,1299,1344,1389,1394,1802,3550,3552,3553,1]

\# HighDSpacing model

\#node_list = [2,3,5,8,36,37,388,663,664,1545,1598,1651,1656,2142,4226,4228,4229,1]

\# LowDSpacingConstMin model

\#node_list =[5,8,9,10,536,539,540,550,551,2122,2530,3489,3492,3493,3549,3552,3553,1]

\# HighDSpacingConstMin model

\#node_list =[5,8,9,10,586,589,590,627,628,2349,2835,4003,4006,4007,4225,4228,4229,1]

\# LowDSpacingConstCol model

\#node_list =[5,8,9,10,536,539,540,550,551,2122,2530,3489,3492,3493,3549,3552,3553,1]

\# HighDSpacingConstCol model

\#node_list $=[5,8,9,10,586,589,590,627,628,2349,2835,4003,4006,4007,4225,4228,4229,1]$

\# Output displacements for each node

for node_num in node_list:

\# Clear/create displacements array

displacements $=[]$

\# Write displacements from the last frame of every step to

\# separate files for each node

for step in step_list:

last_frame $=$ odb.steps[step].frames[-1]

\# Add the value to displacement array

displacements.append(last_frame.fieldOutputs['U'].values[node_num - 1].data[0])

\# Wait to write data to file until last step

if step $==$ last_step:

file_name $=$ './node_displacement/node_' + str(node_num $)+$ '_disp.txt'

fid = open(file_name, 'wb')

for index in range $(0$, len(displacements)):

print $\gg$ fid, displacements[index]

print 'Node ', node_num, ' complete'

fid.close

odb.close

\section{Appendix H: MatLab Curve Fit Code}

The MatLab curve fitting code is composed of the primary file and two functions. The primary

file, TangentDelta.m, gathers the data from the python script created data files and calculates an average 
strain for the periodic composite structure. The first function, CurveFit.m, fits a sinusoidal equation to the data and yields a tangent delta value. The second function, rsquare.m, calculates a coefficient of determination to quantify how well the sinusoidal equation fit the data. The tangent delta, coefficient of determination, strain data, and curve fit are then plotted to figures via the primary MatLab file.

\section{Primary File}

\%TangentDelta.m

$\% \%$ MatLab code to aqcuire data from Abaqus files

$\% \%$ and determine the tangent delta for each analysis

clear all

close all

$\% \%$ Save data from Abaqus displacement files to column vectors

filename $=$ 'node_2_disp.txt';

A1 = importdata(filename);

filename $=$ 'node_3_disp.txt';

A2 = importdata(filename);

filename $=$ 'node_5_disp.txt';

A3 = importdata(filename);

filename $=$ 'node_8_disp.txt';

A4 = importdata(filename);

filename $=$ 'node_34_disp.txt';

A5 = importdata(filename);

filename $=$ 'node_35_disp.txt';

A6 = importdata(filename);

filename = 'node_358_disp.txt';

A7 = importdata(filename);

filename = 'node_611_disp.txt';

A8 = importdata(filename);

filename $=$ 'node_612_disp.txt';

A9 = importdata(filename);

filename $=$ 'node_1422_disp.txt';

A10 = importdata(filename);

filename = 'node_1471_disp.txt';

A11 = importdata(filename); 
filename $=$ 'node_1520_disp.txt';

A12 = importdata(filename);

filename $=$ 'node_1525_disp.txt';

A13 = importdata(filename);

filename $=$ 'node_1972_disp.txt';

A14 = importdata(filename);

filename $=$ 'node_3888_disp.txt';

A15 = importdata(filename);

filename = 'node_3890_disp.txt';

A16 = importdata(filename);

filename = 'node_3891_disp.txt';

A17 = importdata(filename);

$\% \%$ Combine all node displacement column vectors into a single array

Disp_Data $=\operatorname{cat}(2, \mathrm{~A} 1, \mathrm{~A} 2, \mathrm{~A} 3, \mathrm{~A} 4, \mathrm{~A} 5, \mathrm{~A} 6, \mathrm{~A} 7, \mathrm{~A} 8, \mathrm{~A} 9, \mathrm{~A} 10, \mathrm{~A} 11, \mathrm{~A} 12, \mathrm{~A} 13, \mathrm{~A} 14, \mathrm{~A} 15, \mathrm{~A} 16 \ldots$

,A17);

$\% \%$ Determine an average displacement from all the nodes and save to a

$\% \%$ single column vector

Disp_Data $=$ transpose $($ Disp_Data $)$;

Ave_Disp $=$ squeeze $($ mean $($ Disp_Data $))$;

Ave_Disp $=$ transpose(Ave_Disp);

$\% \%$ Calculate the average strain behavior based on periodic unit length

$\% \mathrm{p}=61 \mathrm{e}-3 ; \%$ Low D-Spacing

$\mathrm{p}=67 \mathrm{e}-3 ; \%$ Normal D-Spacing

$\% \mathrm{p}=73 \mathrm{e}-3 ; \%$ High D-Spacing

Data $=$ Ave_Disp/p; \% Divide by the periodic length to get strain

$\% \%$ Remove data from first 10 cycles

Data_10_cycles = Data(201:length(Data) $)$;

$\% \%$ Initialize frequency, time, and initial amplitude

$\mathrm{f}=1 ; \% 1 \mathrm{~Hz}$ frequency

$\% \mathrm{f}=3 ; \% 3 \mathrm{~Hz}$ frequency

$\% \mathrm{f}=9 ; \% 9 \mathrm{~Hz}$ frequency

$\% \mathrm{f}=15 ; \% 15 \mathrm{~Hz}$ frequency

$\% \mathrm{t}=1 /(20 \cdot * \mathrm{f}): 1 /(20 . * \mathrm{f}): 20 / \mathrm{f} ; \%$ Time for entire data

$\mathrm{t}=10 / \mathrm{f}+1 /\left(20 * *_{\mathrm{f}}\right): 1 /(20 * \mathrm{f}): 20 / \mathrm{f} ; \%$ Time for last 10 cycles

$\mathrm{t}=\mathrm{t}(:) ; \%$ Transpose time to match Data vector

$\mathrm{t} 2=0: 1 /\left(20 *{ }^{*}\right): 1 / \mathrm{f}$

initial_amp $=2.265 \mathrm{e}-4$; 
$\% \%$ Function file that accepts curve parameters as inputs and then outputs

$\% \%$ fitting error

$\%$ Starting $=\operatorname{rand}(1,3)$

Starting $=\operatorname{rand}(1,2)$

options = optimset('Display','iter');

\%Estimates=fminsearch (@CurveFit,Starting,options,t,Data,f,initial_amp);

$\%$ Curve fit for entire data

Estimates = fminsearch $(@$ CurveFit,Starting,options,t,Data_10_cycles,f,... initial_amp);

$\%$ Curve fit for last 10 cycles

$\% \%$ Calculate curve fit equation and coefficient of determination strain $=$ Estimates $(1) * \sin \left(2 . *\right.$ pi. $* f^{*} \mathrm{t}-$ Estimates $\left.(2)\right)+$ initial_amp;

[r2 rmse] = rsquare(Data_10_cycles,strain); \% r^2 value for last 10 cycles

$\%$ strain $=$ Estimates $(1) * \sin \left(2 . *\right.$ pi $* * f^{*} \mathrm{t}$ - Estimates $\left.(2)\right)+$ initial_amp;

$\%[\mathrm{r} 2 \mathrm{rmse}]=$ rsquare $($ Data,strain $) ; \% \mathrm{r}^{\wedge} 2$ value for entire data

$\% \%$ Normalized stress and strain history for first cycle

norm_stress $=\sin (2 . *$ pi. $* \mathrm{f} * \mathrm{t} 2)$;

norm_strain $=\sin \left(2 . *\right.$ pi. $* *^{*} * \mathrm{t} 2-$ Estimates $\left.(2)\right)$;

$\% \%$ Plot the fitted curve over the raw data

fig1 = figure;

plot(t,Data_10_cycles,'*') \% Plot last 10 cycles

$\%$ plot(t,Data,'*') \% Plot entire data

hold on

$\operatorname{plot}\left(\mathrm{t}, \mathrm{strain}, \mathrm{r}^{\prime}\right)$

xlabel('Time (seconds)','FontSize',16)

ylabel('Strain (unitless)','FontSize',16)

title('Tangent Delta Calculation','FontSize',16)

str $=\{$ 'R-squared',num2str(r2),'Tangent Delta',num2str(Estimates(2)) $\}$;

annotation('textbox',[.7,.12,.2,.15],'String',str);

set(fig1,'Position',[1 540500 400])

$\% \%$ Plot normalized stress and strain for 1 cycle on a separate figure

fig2 = figure;

plot(t2,norm_stress,'--r')

hold on

plot(t2,norm_strain,'k')

set(fig2,'Position',[1 1500 400])

\section{Curve Fit Function}

$\%$ CurveFit.m

function sse $=$ CurveFit(params,Input,Actual_Output,f,initial_amp)

amplitude $=\operatorname{params}(1)$; 
delta $=\operatorname{params}(2)$

Fitted_Curve $=$ amplitude. $* \sin ((2 . * p i . * f) *$ Input - delta $)+$ initial_amp;

Error_Vector $=$ Fitted_Curve - Actual_Output;

$\% \%$ When curvefitting, a typical quantity to minimize

$\% \%$ is the sum of squares error

sse $=\operatorname{sum}\left(\right.$ Error_Vector. $\left.{ }^{\wedge} 2\right)$;

\section{$\underline{\text { Coefficient of Determination Function }}$}

$\%$ rsquare.m

function $[\mathrm{r} 2 \mathrm{rmse}]=\operatorname{rsquare}(\mathrm{y}, \mathrm{f}, \mathrm{varargin})$

$\%$ Compute coefficient of determination of data fit model and RMSE

$\%$

$\%[\mathrm{r} 2 \mathrm{rmse}]=\operatorname{rsquare}(\mathrm{y}, \mathrm{f})$

$\%[\mathrm{r} 2 \mathrm{rmse}]=\operatorname{rsquare}(\mathrm{y}, \mathrm{f}, \mathrm{c})$

$\%$

$\%$ RSQUARE computes the coefficient of determination (R-square) value from

$\%$ actual data $\mathrm{Y}$ and model data $\mathrm{F}$. The code uses a general version of

$\% \mathrm{R}$-square, based on comparing the variability of the estimation errors

$\%$ with the variability of the original values. RSQUARE also outputs the

$\%$ root mean squared error (RMSE) for the user's convenience.

$\%$

$\%$ Note: RSQUARE ignores comparisons involving NaN values.

$\%$

$\%$ INPUTS

$\%$ Y : Actual data

$\%$ F : Model fit

$\%$

$\%$ OPTION

$\% \quad \mathrm{C} \quad$ : Constant term in model

$\% \quad$ R-square may be a questionable measure of fit when no

$\% \quad$ constant term is included in the model.

$\%$ [DEFAULT] TRUE : Use traditional R-square computation

$\% \quad$ FALSE : Uses alternate R-square computation for model

$\% \quad$ without constant term $[\mathrm{R} 2=1-\mathrm{NORM}(\mathrm{Y}-\mathrm{F}) / \mathrm{NORM}(\mathrm{Y})]$

$\%$

$\%$ OUTPUT

$\%$ R2 : Coefficient of determination

$\%$ RMSE : Root mean squared error

$\%$

$\%$ EXAMPLE

$\% \quad \mathrm{x}=0: 0.1: 10$

$\% \quad \mathrm{y}=2 . * \mathrm{x}+1+\operatorname{randn}(\operatorname{size}(\mathrm{x}))$;

$\% \mathrm{p}=\operatorname{polyfit}(\mathrm{x}, \mathrm{y}, 1)$;

$\% \quad \mathrm{f}=\operatorname{polyval}(\mathrm{p}, \mathrm{x})$;

$\% \quad[\mathrm{r} 2 \mathrm{rmse}]=\operatorname{rsquare}(\mathrm{y}, \mathrm{f})$;

$\%$ figure; $\operatorname{plot}\left(\mathrm{x}, \mathrm{y},{ }^{\prime} \mathrm{b}-\mathrm{\prime}\right)$; 


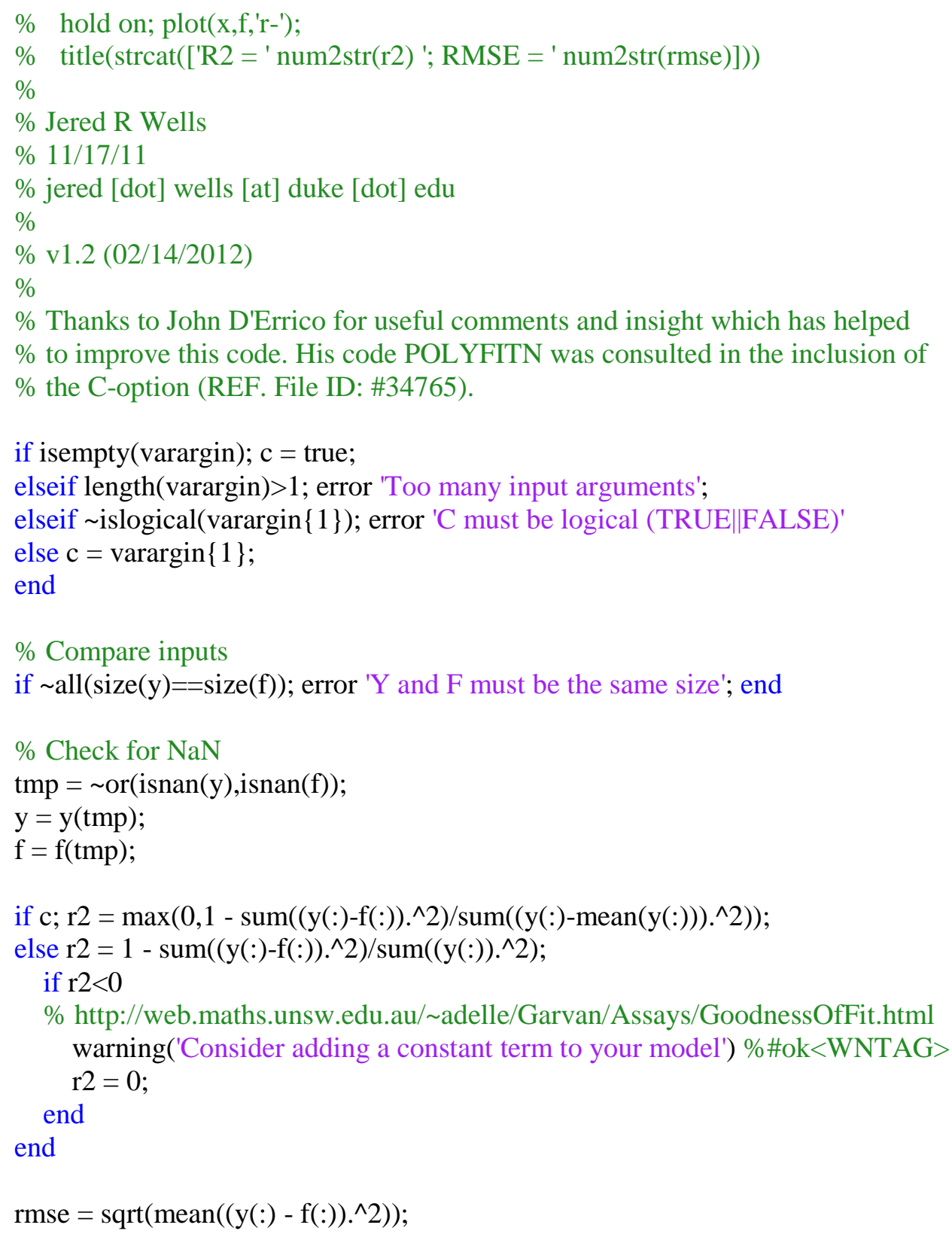

